\title{
High-resolution molecular stratigraphy of Oligocene-Miocene sequence of Tumaco-1-ST-P well, Tumaco forearc Basin, Colombia
}

\author{
J. E. Cortes ${ }^{1,3,5} \cdot$ A. Mejía-Molina ${ }^{2,6} \cdot$ C. A. Vargas ${ }^{3} \cdot$ S. I. Cortes ${ }^{4}$
}

Received: 1 August 2018 / Accepted: 8 April 2019 / Published online: 16 April 2019

(c) The Author(s) 2019

\begin{abstract}
In the present work, high-resolution molecular stratigraphy technique has been applied to the rock samples from Tumaco1-ST-P well to investigate vertical variations of the deposited organic matter (O.M.) based on screening parameter and high-resolution biomarkers in both saturate and aromatic fractions in order to establish its origin, organic facies, depositional paleoenvironment, thermal maturity, biodegradation processes and to evaluate the exploratory potential in this frontier forearc basin. The O.M. is immature with a high concentration of biolipids (early diagenesis) and with poor to fair generation potential originated from a type II/III or III kerogen. Original total organic carbon $\left(\mathrm{TOC}_{\mathrm{o}}\right.$ ) and original hydrocarbon index $\left(\mathrm{HI}_{\mathrm{o}}\right)$ values for each formation and lithology are determined to evaluate the hydrocarbons potential in the basin. $n$-alkanes, isoprenoids, and tricyclic terpanes suggest an algal/bacterial and terrestrial input deposited on a marine-deltaic shale under oxic to suboxic conditions. The O.M. was deposited on siliciclastic rocks in a marine-continental (transitional) environment, although carbonate rocks were also deposited in the bottom of the column (late Oligocene). The molecular stratigraphic interpretation allows to conclude that organic facies and lithology in Tumaco-1-ST-P are characterized in Cascajal and Tangareal del Mira Fms. (1000-5000 ft) by a marine-deltaic paleoenvironment with an important terrestrial contribution and varying between deltaic plain and prodelta during the late Miocene. At the top of Tumaco Fm (5000-9000 ft) in the late to medium Miocene, the depositional environment was like the marine delta of the Tangareal del Mira Fm, changing to a marine platform environment at 8000 to $10,000 \mathrm{ft}$ depth in the middle to early Miocene. Toward the bottom of the column $(10,000-12,000 \mathrm{ft})$, Tumaco Fm was deposited in the late Oligocene under a marine shelf environment. In the present paper, we propose a modeling approach, based on the evolution of the thermal maturity through the stratigraphic column, that at 17,000 feet depth could exist rocks with O.M. in the hydrocarbon generation window for Tumaco Basin, Colombia.
\end{abstract}

Keywords Hydrocarbon source rock · Marine-deltaic environment · Thermal maturity · Saturate-aromatic biomarkers . Frontier basin · Tumaco forearc basin

\section{Introduction}

$\bowtie$ J. E. Cortes

jcortes@petromarkers.com; jecortesl@unal.edu.com

1 Antek S.A. Geoscience Technology Center, Calle 25B \#85B-54, Bogotá, Colombia

2 National Hydrocarbon Agency, ANH. Av. Calle 26 No 59-65, 2nd Floor, Bogotá, Colombia

3 Universidad Nacional de Colombia, Carrera 45 No 26-85, Bogotá, DC, Colombia

4 Geoscience Department, Southern Methodist University, 6425 Boaz Ln, Dallas, TX 75205, USA

5 Present Address: Petromarkers, Inc, 1815 County Rd 130, No 6, Pearland, TX 77581, USA

6 Present Address: Yachay Tech University, Urcuquí, Ecuador
Tumaco basin is a frontier basin under the influence of a subduction zone of the Pacific active margin. Consequently, the importance of both the new stratigraphic and the geochemical data is essential to provide new information for developing new exploration projects in the Colombian Pacific margin. The Tumaco-1-ST-P stratigraphic well gives evidences of the Tumaco basin sedimentation system during the late Oligocene to Pliocene, dominated by the movements of the paleodeltas of the Tumaco and Mira rivers (ANH-Universidad de Caldas 2011, ANH-Antek S.A. 2013). In the late Oligocene-middle Miocene, Tumaco Fm is observed the presence of a large deltaic complex (associated with paleodelta of the Patia River), with sources areas 
that involve, at least partially, the Central Cordillera. For the late Miocene-early Pliocene, the displacement to the north of the Patía River leads to the establishment of the Mira fluvio-deltaic system in the area, represented by deposits of the Tangareal del Mira Fm, which is the only present source areas of the Western Cordillera. Finally, in the late Pliocene, deltaic deposits of Cascajal Fm record a period of intense volcanism (ANH-Antek S.A. 2013).

The application of high-resolution organic geochemical techniques to evaluate the sedimentary sequence on a "layerby-layer" basis is called "molecular stratigraphy" (Brassell et al. 1986; Farrimond et al. 1990; Keely et al. 1993). In this study, this approach was applied to investigate the evolution of the sedimentary organic matter in the Tumaco-1-ST-P stratigraphic well in the range between 750 and $11,930 \mathrm{ft}$ covering the Miocene-Oligocene sequence in the onshore Tumaco basin, Colombia to characterize the origin and type of organic matter, paleodepositional environment, genetic relation between bitumen, maturity, and possible degradation processes in a sedimentary basin under the subduction influence.

\section{Geological setting}

\section{Regional stratigraphy}

The onshore Tumaco basin is located in the southwestern of Colombia. The basin is bounded by the Garrapatas fault system in the north, Ecuador country in the south, Western Cordillera (uplift) in the east, and the Pacific Ocean in the west (IGAC-Ingeominas 2001; Barrero et al. 2007; Cediel et al. 2010; ANH-Universidad de Caldas 2011; Borrero et al. 2015). The Tumaco forearc basin contains a pre-Miocene mega-sequence and three post-lower Miocene sequences deposited in shelf to bathyal environments. The basin basement is made up of Cretaceous metasedimentary and volcanic rocks (Cediel et al. 2010). The study area map and detailed stratigraphy column of the Tumaco-1-ST-P well are given in Figs. 1 and 2, respectively (ANH-Antek S.A. 2013).

Previous work including a detailed discussion of the geology of the onshore Tumaco basin is found in the literature derived from recent projects led by National Hydrocarbon Agency, Colombia (Universidad de Caldas 2011; ANHAntek S.A. 2013). Suárez (2007) studied the Colombian

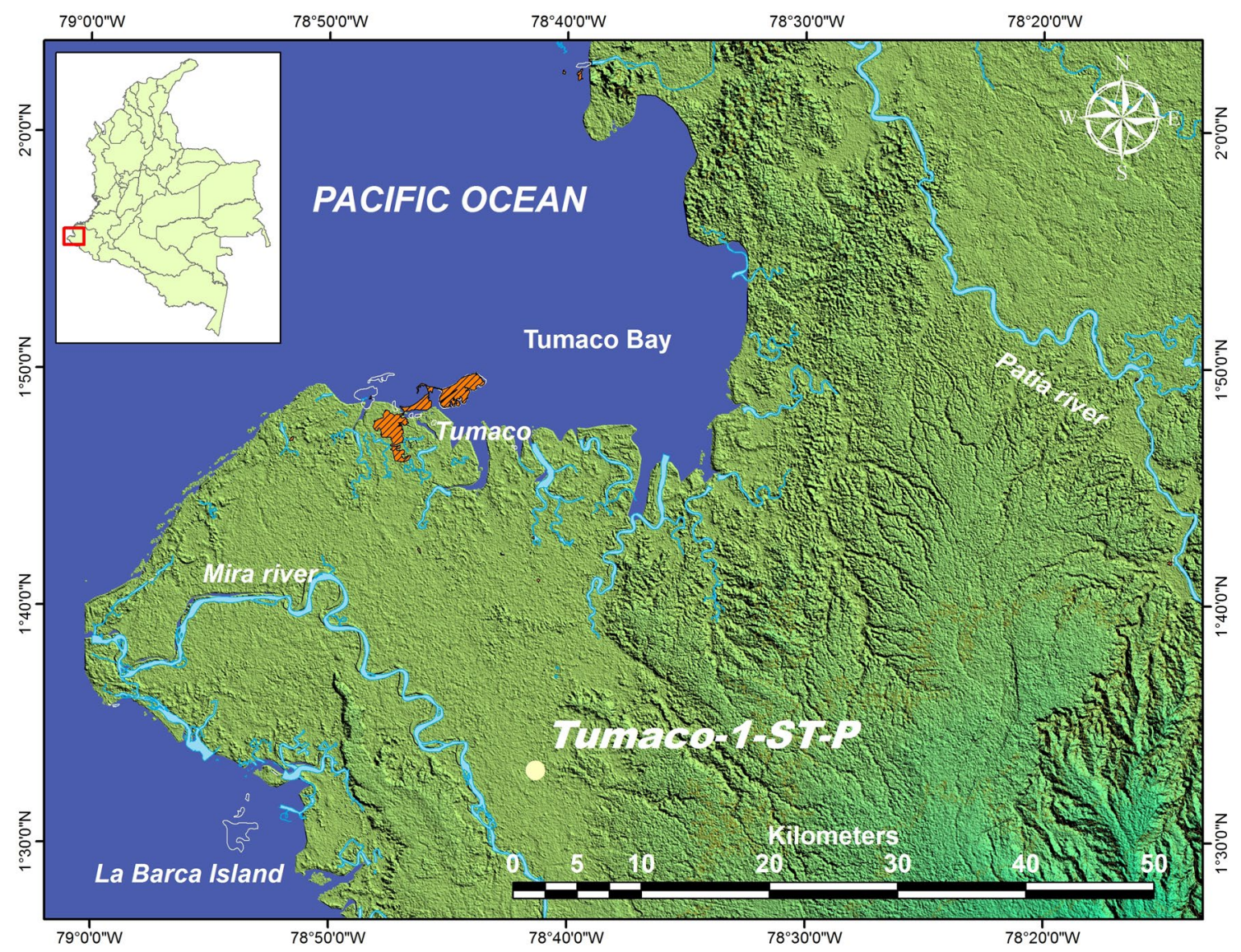

Fig. 1 Map showing the study area and location of the Tumaco-1-ST-P stratigraphic well (ANH-Antek S.A. 2014) 
Fig. 2 Generalized lithostratigraphic column of Tumaco basin (ANH-Antek S.A. 2014)
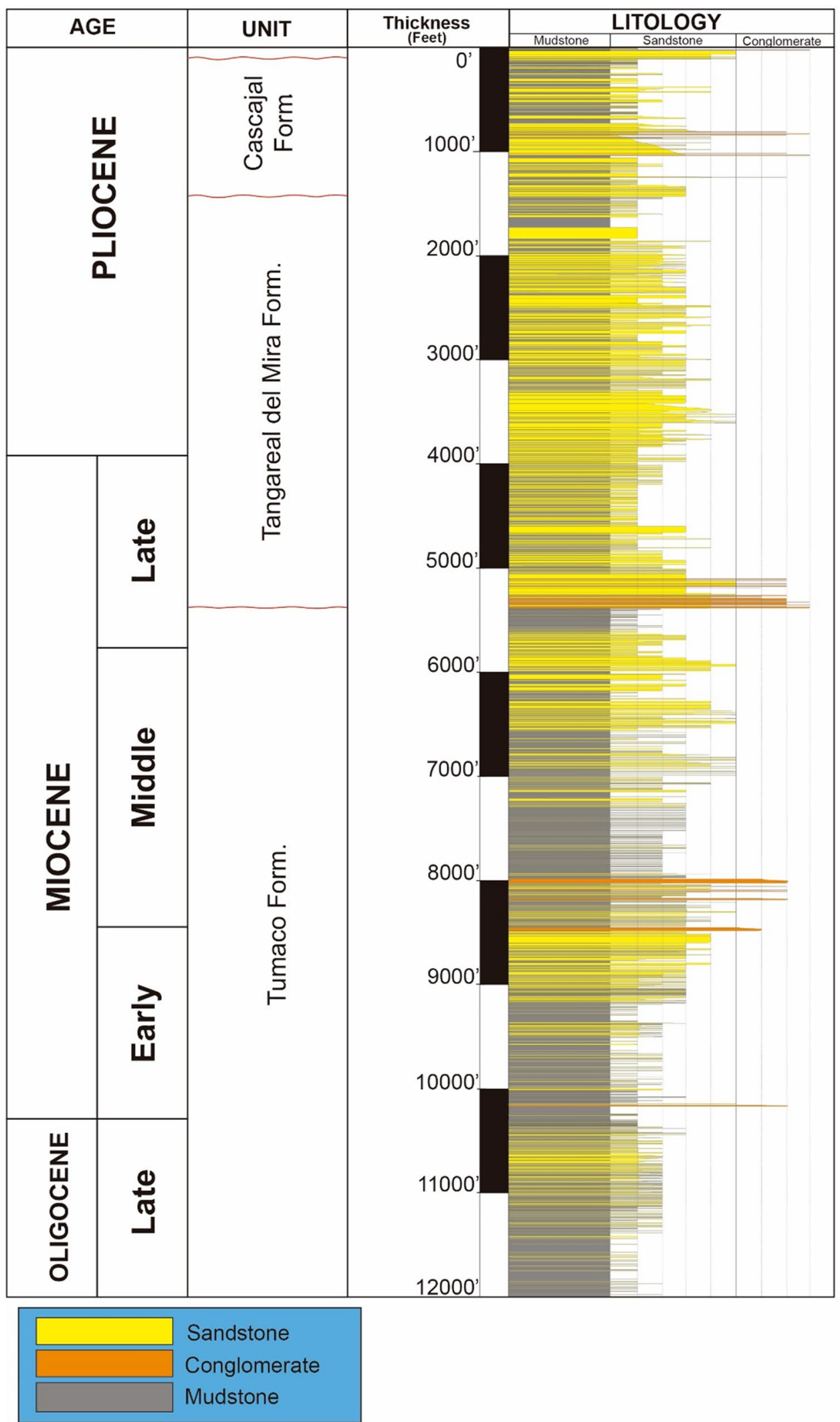
Pacific provinces using a data set including seismic, gravimetric, and aeromagnetometric data. Additional geological and tectonic data can be searched in publications by Suárez (2007), Echeverri et al. (2011), and Echeverri et al. (2015). Stratigraphic studies of the area have been published by Cediel et al. (2010) based on the description of the Chaguii-1, Majagua-1 y Remolino Grande-1 stratigraphic wells.

In brief, the basement of the Tumaco basin is interpreted as an allochthonous block composed of fragments of oceanic plateau (Kerr et al. 2002) and the Santonian-Campanian arc of islands, accreted to the continent during the late Cretaceous and early Paleocene (ANH-Universidad de Caldas 2011). The following discussion about geological setting and regional stratigraphy is taken from the technical report presented by Antek S.A. to the National Hydrocarbon Agency (NHA), Colombia (ANH-Antek S.A. 2013).

The Tumaco formation (Chatiense-early Tortoniense) is composed of interbedded mudstones, arenite, and occasionally conglomerates covering the bottom of Tumaco 1-ST-P well (interval 11,980-5381 ft), which has a thickness greater than the $6599 \mathrm{ft}$ (the base is not present). The rocks of this unit can be grouped into eight facies associations represented by bar and estuarine channels, mouth bar, coastal lagoon, proximal front deltaic, prodelta, and platform deposits, respectively.

In the facies associated with the Tumaco Fm, occur occasional mudstones, sandstones and conglomerates, abundant fossils of marine affinity and evidence of rocks associated with deltaic paleoenvironment with the influence from the continent, it allows considering the existence during the late Oligocene and much of the Miocene of an enormous deltaic complex dominated by waves and tides. It seems to correspond to the paleodelta of the Patia River when it disembogued in the area now occupied by the Tumaco Bay.

The Tumaco Fm origin indicates an input area that, at least partially, involved sectors of the Central Cordillera that were recorded in the stratigraphic record with the presence of minerals such as garnets and pumpellyite and fragments of metamorphic rocks such as marbles, although there is a clear preponderance of a mafic-intermediate source area and some recycled rocks of the first cycle.

The Tangareal del Mira Fm (middle Tortoniense-Zancliense), rests unconformably on the Tumaco Fm covering the interval between 1437 and $5381 \mathrm{ft}$ (thickness $3944 \mathrm{ft}$ ). In general, the unit consists of interbedded lithic sandstones and mudstones with a development of conglomerates at the base that can be grouped into five associations of facies representing river channels, bar and fluvial channels, bay estuarine, prodelta, and front deltaic deposits.

As in Tumaco Fm, the set of facies and facies associations of Tangareal del Mira Fm allow us to observe the presence of abundant fossils of marine affinity and evidence of continental contribution that would support the existence of a deltaic complex dominated by waves and tides occurs with migration north Mira River in the late Miocene, the position left to the Patia River Delta in its movement to its present site mouth. The development of fluvial facies at the base of the unit, showing the relative fall of sea level in the area, which is succeeded by two transgressive-regressive greater cycles, where is evident a high volcanic influence.

The Cascajal formation (Zancliense), which was examined in detailed in Tumaco-1-ST-S well, since in Tumaco1-ST-P well, only cuttings were available and partially in some outcrops located on the Tumaco Bay; it is composed of interbedded mudstones, litoarenites, and conglomerate facies, locally bioturbated, it reposes unconformably on the Tangareal del Mira Fm. Moreover, it may be characterized in five associations of facies represented by the proximal deltaic front, distal deltaic front, channels, and estuarine bars, mouth bar, and prodelta deposits (ANH-Universidad de Caldas 2011; ANH-Antek S.A. 2013).

The facies associated with Cascajal formation allow to observe the presence of bioturbated segments, abundant fossils of marine affinity and the continental influence that would support the existence during the late Pliocene of a deltaic complex dominated by waves and tides (Fig. 4) which is associated with the progradation of the delta of Mira River. In this unit, the volcanic contribution is even greater than in the Tangareal del Mira.

The Quaternary is represented by the first $110.89 \mathrm{ft}$ of Tumaco 1-ST-S well (ANH-Universidad de Caldas 2011), which corresponds to fluvial deposits of the Mira River. These deposits consist of interbedded litoarenites and sublitoarenites, massive mudstones, and occasional interbedded polymictic conglomerates with a significant contribution of volcanic rocks.

\section{Samples and experimental}

\section{Samples}

Samples were selected from the stratigraphic column in the interval 750 to $12,000 \mathrm{ft}$ covering the Miocene to the late Oligocene in the Tumaco-1-ST-P well. Two hundred and fifty samples were analyzed for TOC and RE-Py, 25 samples for vitrinite reflectance, and 100 samples for extractable organic matter (EOM), SARA fractionation, GC/FID, and GCMS analyses applying Antek S.A. geochemical validated procedures, which are part of its ONAC-ISO-17025 quality assurance manual (Antek S.A. 2011). All the samples were picked and washed to remove any contaminant. After the samples had been air-dried or oven-dried, they were crushed on porcelain mortar and pestle up to $62 \mu \mathrm{m}$ for screening analysis, organic matter extraction, and biomarkers analysis. 


\section{Total organic carbon (TOC) and Rock-Eval pyrolysis (RE-Py)}

For TOC analysis, $1 \mathrm{~g}$ of rock and cutting powder were digested overnight with $10 \% \mathrm{HCl}$ to remove the carbonate, then washed and dried in oven at $60^{\circ} \mathrm{C}$ for $24 \mathrm{~h}$. Approximately $100 \mathrm{mg}$ of sample was transferred onto a Leco cell and mixed with copper/iron catalyst. TOC analysis was run on a Leco S-200 carbon analyzer. Rock-Eval pyrolysis analysis was done in a Vinci-VI instrument with temperature programmed from 30 to $600{ }^{\circ} \mathrm{C}$ at $30{ }^{\circ} \mathrm{C} / \mathrm{min}$ rate. Helium was maintained at $60 \mathrm{~mL} / \mathrm{min}$. S1, S2 peaks and $T_{\max }$ were detected by FID detector, while S3 peak was quantitated by TCD detector.

\section{Organic petrography (vitrinite reflectance)}

All samples presented a relatively poor dispersed organic matter that it was necessary to make a organic matter concentration with zinc chloride ( $\mathrm{ZnCl} 2)$, a heavy liquid prepared at a density of near $2 \mathrm{~g} / \mathrm{mL}$ (ICCP 1963; ISO 1985, 1994; Pawlewicz 2013). Vitrinite reflectance analysis was conducted according to ASTM D-7708-11 and ISO-7404 standard methods. The measurements were done using a Carl Zeiss model Axio-Imager-100 petrographic microscope equipped with reflected and fluorescence UV light in the spectral range between 200 and $3000 \mathrm{~nm}$. The microscope was coupling to a Craic model CoalPro spectrophotometer and a high-speed high-resolution photographic camera. The system was calibrated using a $0.4,0.9,1.7$, and $5.3 \%$ synthetic standards. The microphotometer was equipped with an oil immersion objective (X50/0.85). The oil immersion used has a refraction index of 1.5189 at $23{ }^{\circ} \mathrm{C}$ over sodium light at $589.3 \mathrm{~nm}$. The reflectance was measured with normal incident monochromatic light at wavelength of $546 \mathrm{~nm}$.

\section{Organic matter extraction and fractionation (EOM and SARA analyses)}

Rock powder samples were extracted using a Dionex ASE200 accelerated extraction equipment. The solvent was $100 \%$ DCM. The extraction condition were solvent pressure at $1500 \mathrm{psi}$, extraction cell temperature of $100{ }^{\circ} \mathrm{C}$, static pressure time of $2 \mathrm{~min}$, solvent flush $75 \%$, and nitrogen purge time of $60 \mathrm{~s}$. After extraction, the solvent was removed until almost dried by rotaevaporation in a Buchi model 130 rotaevaporator and then transferred in a $4 \mathrm{~mL}$ pre-weighted vial. Finally, the extracts were dried with a nitrogen stream and the extractable organic matter (EOM) was determined by gravimetry.

Due to the low EOM content, all the bituminous extracts were used for SARA fractionation by microcolumnation on alumina. The bituminous extracts were dissolved in an excess of n-pentane, and the asphaltenes were precipitated overnight at $-10{ }^{\circ} \mathrm{C}$ into the refrigerator. Asphaltenes were separated from maltene fraction by centrifugation. The maltene was fractionated by liquid chromatography on activated silica gel in saturates, aromatics, and resins. The saturated fraction was eluted with $n$-hexane; the aromatic fraction was eluted with $n$-hexane: dichloromethane (70:30), and the polar (NSO) fraction was eluted with dichloromethane: methanol (98:2). The saturated fraction was analyzed by GC/ FID. The branched and cyclic fractions (biomarkers) were separated from $n$-alkanes by silicalite powder S-115 and then analyzed by SIM-GC/MS. The aromatic fractions were analyzed directly by GC/FID, and some selected samples were analyzed for aromatic biomarkers by gas chromatography-mass spectrometry (SIM-GC/MS).

\section{$\mathrm{C}_{15}{ }^{+}$saturated fraction by GC/FID}

$\mathrm{C}_{15}{ }^{+}$saturated fractions obtained from SARA analysis were dissolved according to the mass with $n$-hexane (approximately $10 \mathrm{mg} / \mathrm{mL}$ ) and analyzed using an Agilent 6890 HRGC/FID fitting with a DB-1 $60 \mathrm{~m} \times 0.25 \mathrm{~mm} \times 0.25 \mu \mathrm{m}$ capillary column. Samples were injected in splitless mode. The GC oven temperature was programmed from 60 to $320{ }^{\circ} \mathrm{C}$ at $5{ }^{\circ} \mathrm{C} / \mathrm{min}$. Helium was used as carrier gas at a linear velocity of $50 \mathrm{~cm} / \mathrm{s}(12 \mathrm{psig})$. Injector/detector was maintained at $320{ }^{\circ} \mathrm{C} / 350{ }^{\circ} \mathrm{C}$, respectively. Data handling was done with Agilent Chemstation version 10 chromatographic software.

\section{Biomarkers by GC/MS-SIM}

SIM-GC/MS of branched and cyclic and aromatic hydrocarbons was analyzed using an Agilent 7890B gas chromatograph coupling to an Agilent 5975C VL MSD mass spectrometer. The separation was carried out using a Restek Rtx-5MS of $60 \mathrm{~m} \times 0.25 \mathrm{~mm} \times 0.25 \mu \mathrm{m}$ capillary column operated at constant flow $(1 \mathrm{~mL} / \mathrm{min}$ ) (initial head column pressure of $8.23 \mathrm{psi}$ ) and the linear velocity of $20 \mathrm{~cm} / \mathrm{s}$. Column program started at $60{ }^{\circ} \mathrm{C}(2 \mathrm{~min})$ to $320^{\circ} \mathrm{C}$ at $3{ }^{\circ} \mathrm{C} / \mathrm{min}$ and maintained at $320{ }^{\circ} \mathrm{C}$ for $11{ }^{\circ} \mathrm{C} / \mathrm{min}$. The ion source was operated in electronic impact (EI) mode at $70 \mathrm{eV}$ at $150^{\circ} \mathrm{C}$. The MS was operated in selective ion monitoring (SIM) for the following saturate ions: internal standard, Squalane $(\mathrm{m} / \mathrm{z}$ 99 and 113$) ; n$-alkanes $(\mathrm{m} / \mathrm{z}, 99)$; isoprenoids $(\mathrm{m} / \mathrm{z}, 183)$, terpanes $(\mathrm{m} / \mathrm{z}$ 191), demethylated hopanes $(\mathrm{m} / \mathrm{z}, 177)$, steranes $(\mathrm{m} / \mathrm{z} 217$ and 218) and aromatic ions: alkylnaphthalenes $(\mathrm{m} / \mathrm{z} 128,142,156,170)$, alkylphenanthrenes $(\mathrm{m} / \mathrm{z}, 178)$, alkyldibenzothiophenes $(\mathrm{m} / \mathrm{z}, 184)$, monoaromatic steroids $(\mathrm{m} / \mathrm{z}$ 253), and triaromatic steroids $(\mathrm{m} / \mathrm{z}, 253)$. The Agilent ChemStation version A02.00 (2012) was utilized as data acquisition system. Biomarker quantitation was done using the area peaks from the GC/MS traces.

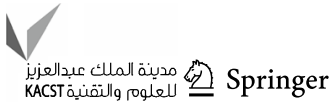




\section{Results and discussions}

\section{Total organic carbon (TOC) and Rock-Eval pyrolysis}

TOC and Rock-Eval pyrolysis results are summarized in Table 1. TOC content analyzed by Leco carbon analyzer, after removal of carbonate minerals, showed values ranging between 0.09 and $2.30 \mathrm{wt} \%$. The major TOC values were found in the deepest intervals of the Tumaco-1-ST-P well $(11,610-11,700 \mathrm{ft})$, which were deposited on calcareous and siliceous siltstones corresponding to the late Oligocene sequence. $47 \%$ of the samples analyzed are grouped in the range between 0.5 and $1.0 \mathrm{wt} \%$, which are considered with fair source rock potential. 38\% of the samples are classified as O.M. with good quality. Finally, $13 \%$ of the total of 250 rock samples analyzed showed TOC values $<0.5 \%$. Rocks organically poor with TOC $<0.5 \%$ are considered, in almost all the cases, like rocks with poor generator potential (Bordenave 1993; Peters and Cassa 1994; Hall et al. 2016) therefore, without economic interest.

Free hydrocarbons (S1 peak) average value was $0.075 \mathrm{mg}$ $\mathrm{HC} / \mathrm{g}$ rock (ranging between 0.02 and $0.80 \mathrm{mg} \mathrm{HC/g}$ rock). The high heterogeneity of $\mathrm{S} 1$ through the stratigraphic column is due to the multiple and successive changes of organic facies in the sedimentary strata. Samples collected at 11,570-11,730 $\mathrm{ft}$ in the late Oligocene displayed the highest values of S1 ranging between 0.2 and $0.8 \mathrm{mg} \mathrm{HC} / \mathrm{g}$ rock, while most of the samples had values $<0.25 \%$, which classifies the generation potential as "poor and/or acceptable" (Peters and Cassa 1994). In practical terms, the peak $\mathrm{S} 1$ is less than the removable fraction (EOM) for immature sediments; for mature sediments, peak S1 and the content of EOM fraction are fairly comparable (Peter et al. 2005).

S2 values vary between 0.07 and 1 or removal $0.45 \mathrm{mg}$ $\mathrm{HC} / \mathrm{g}$ rock, with an average of $1.1 \pm 1.36 \mathrm{mg} \mathrm{HC} / \mathrm{g}$ rock. The highest S2 peak values were found in the interval around $11,700 \mathrm{ft}$ in the late Oligocene of the Tumaco Fm. S2 peak at $5570 \mathrm{ft}$ registered a value of $10.45 \mathrm{mg} \mathrm{HC} / \mathrm{g}$ rock which is inconsistent with the low thermal evolution of the O.M. in the samples, based on the values of TOC, S1 and bitumen extracted (EOM). The value of $T_{\max }$ is proportional to the thermal maturity of the organic matter in a rock (Tissot and Welte 1984). $T_{\max }$ values vary between 328 and $445^{\circ} \mathrm{C}$, with an average value of $417.5^{\circ} \mathrm{C}\left(\mathrm{SD} \pm 26.23{ }^{\circ} \mathrm{C}\right)$, indicating rocks relatively immature or at the start of its early maturity.

Present-day hydrogen index $\left(\mathrm{HI}_{\mathrm{pd}}\right)$ suggests that a significant number of samples from the Tumaco-1-ST-P well corresponds to type II/III kerogen, characterized by $\mathrm{HI}_{\mathrm{pd}}$ between 300 and $600 \mathrm{mg} \mathrm{HC} / \mathrm{g}$ TOC. This kind of kerogen is dominated by liptinitic macerals, despite a minor vitrinite and inertinite input. Type II/III kerogen describes mixtures of transitional marine and terrestrial organic matter.
Figure 3 displays the hydrogen index $\left(\mathrm{HI}_{\mathrm{pd}}\right)$ versus oxygen index $\left(\mathrm{OI}_{\mathrm{pd}}\right)$ cross-plot to illustrate the quality and level of maturation of the studied samples. Results cover a broad range of maturity between oil-prone type II kerogen with $\mathrm{HI}_{\mathrm{pd}}$ of $525 \mathrm{mg} \mathrm{HC/g}$ TOC and dry gas-prone type IV kerogen with $\mathrm{HI}_{\mathrm{pd}}$ of $13 \mathrm{mg} \mathrm{HC} / \mathrm{g}$ TOC values, indicating that the O.M. in study comes from a single and unique kerogen with a significant variation of the organic facies and paleodepositional environments.

Figure 4 shows the cross-plot $\mathrm{HI}_{\mathrm{pd}}$ versus $T_{\max }$ to correlate kerogen type and thermal maturity in terms of the degree of kerogen conversion, indicating that most of the samples show a $T_{\max }<430{ }^{\circ} \mathrm{C}$ and $\mathrm{HI}_{\mathrm{pd}}<600 \mathrm{mg} \mathrm{HC} / \mathrm{g}$ TOC confirming an immature O.M. and type III kerogen. Some specimens, however, show an O.M. with oil generation potential and type II and II/III kerogen. The pseudo van Krevelen diagram between $\mathrm{S} 2$ and $\mathrm{TOC}_{\mathrm{pd}} \%$ indicating the type and quality of the kerogen in the rock samples is shown in Fig. 5 (Langford and Blanc-Valleron 1990). Thus, a large number of rocks and cuttings from Tumaco-1-ST-P well confirm a type III and/or IV kerogen (gas prone and dry gas prone, respectively). About 20 samples showed a transitional type II/III kerogen, which would generate oil/gas, and a smaller number of samples presented a type II kerogen (oil prone, usually marine). Thus, Cascajal and Tangareal del Mira formations show the lower quality due to its terrestrial origin and its diagenetic maturity.

Figure 6 shows the paleoenvironmental evolution based on the lithology and stratigraphic analyses for Tumaco-1-STP well (ANH-Antek S.A.S 2013). Since the kerogen type is related to the original type of organic matter and its evolution and preservation over time, which is in turn associated with depositional environment and climate (Hall et al. 2016), the evaluation of depositional environment can be done through the TOC content and RE-pyrolysis data and indexes. Thus, the results discussed in Sect. 4.1 indicate that O.M. was deposited in a fluvio-deltaic and deltaic (upper delta plain/lower delta plain and prodelta) environment on siliciclastic lithofacies with organofacies characterized by input of higher plants, algae, and marine algae on a II/III kerogen type which is consistent with the depositional environment deduced from lithology and stratigraphic analysis.

\section{Vitrinite reflectance and maceral analysis}

Organic petrography is used almost systematically as a complement of the screening parameters (TOC, RE-Py, Fluorescence, etc.) to evaluate the O.M. type and the characteristics of the depositional environment, such as redox conditions (oxic/anoxic), energy level, microorganisms type, and additional data that allows the quantification of the thermal evolution of the O.M. Mean random Vitrinite reflectance (Rv,r) of the dispersed organic matter was measured on immersed 


\begin{tabular}{|c|c|c|c|c|c|c|c|c|c|c|c|c|c|c|c|}
\hline 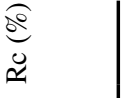 & 1 & 1 & 1 & 1 & 1 & 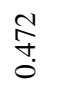 & 吕 & 1 & 1 & 1 & 1 & 1 & 1 & 1 & 1 \\
\hline$\approx @$ & 1 & 1 & 1 & 1 & $\frac{m}{m}$ & 1 & 1 & 1 & 1 & 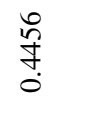 & 1 & 1 & 1 & 1 & 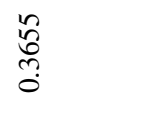 \\
\hline$\stackrel{2}{\circ}$ & $a$ & 0 & $\vec{\sim}$ & $\tilde{\imath}$ & ஜे & ஜे & 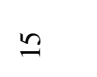 & $\nabla$ & $r$ & $\infty$ & $=$ & $a$ & $\simeq$ & $\simeq$ & $\sigma$ \\
\hline 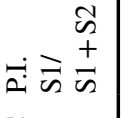 & $\stackrel{8}{0}$ & $\stackrel{8}{\circ}$ & $\vec{\jmath}$ & $\stackrel{\overbrace{}}{0}$ & ஜ̊. & ஜి. & $\stackrel{n}{0}$ & $\stackrel{t}{0}$ & $\stackrel{0}{0}$ & $\stackrel{\infty}{\circ}$ & $\vec{J}$ & $\stackrel{8}{0}$ & $\stackrel{2}{0}$ & $\stackrel{7}{\circ}$ & $\stackrel{8}{0}$ \\
\hline 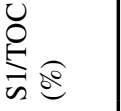 & $\stackrel{m}{+}$ & $\stackrel{\circ}{\circ}$ & $\stackrel{\circ}{\ddot{2}}$ & $\begin{array}{l}\dot{e} \\
i n\end{array}$ & $\stackrel{R}{i}$ & $\stackrel{o}{q}$ & $\stackrel{0}{\infty}$ & $\stackrel{\substack{++}}{ }$ & $\stackrel{\circ}{\varrho}$ & $\stackrel{m}{\infty}$ & $\stackrel{n}{n}$ & $\stackrel{\check{\Xi}}{\circ}$ & $\ddot{n}$ & 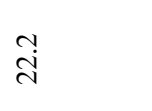 & $\stackrel{+}{\stackrel{ \pm}{\Xi}}$ \\
\hline 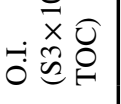 & $\stackrel{8}{0}$ & 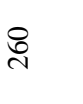 & $\underset{\sim}{~}$ & 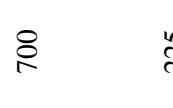 & ָิ & ిీ & $\stackrel{\infty}{\sim}$ & $\stackrel{\oplus}{-}$ & $\stackrel{\widetilde{\infty}}{\sim}$ & $\stackrel{\varrho}{\Xi}$ & $\stackrel{\Re}{\varrho}$ & $\vec{\Xi}$ & $\stackrel{\sim}{\sim}$ & లె & ల్ \\
\hline 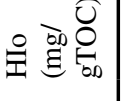 & F & $\Xi$ & 8 & Фે & $\stackrel{2}{2}$ & $\vec{m}$ & in & $\vec{\Xi}$ & $\stackrel{g}{g}$ & હે & ஜ & $\stackrel{\infty}{\ominus}$ & $\stackrel{m}{=}$ & 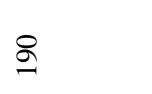 & 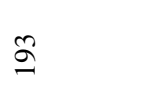 \\
\hline 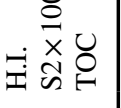 & $\mathscr{F}$ & 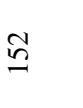 & in & $\stackrel{\infty}{=}$ & 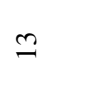 & $\tilde{N}$ & fo & $\hat{a}$ & ले & $\widehat{\widehat{\Xi}}$ & $\hat{\sim}$ & $\stackrel{0}{\sigma}$ & $\stackrel{\infty}{=}$ & $\stackrel{?}{I}$ & $\stackrel{\infty}{\subseteq}$ \\
\hline 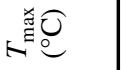 & $\overrightarrow{\mathrm{f}}$ & ల్లి & 怘 & $\aleph_{m}^{\infty}$ & $\ddot{q}$ & $\underset{\Im}{\stackrel{J}{J}}$ & Э & $\overrightarrow{\mathrm{f}}$ & లై & $\stackrel{m}{m}$ & बे & $\underset{\sim}{\infty}$ & d্ల & : & $m$ \\
\hline$\approx \stackrel{U_{0}}{\Xi}$ & $\stackrel{⿱ 艹 冖}{\rightarrow}$ & $\stackrel{m}{-}$ & $\stackrel{f}{J}$ & $\stackrel{0}{0}$ & $\stackrel{9}{\leftrightarrows}$ & $\hat{a}$ & $\hat{m}$ & $\stackrel{\text { ㄱ. }}{i}$ & $\stackrel{n}{b}$ & $\stackrel{m}{m}$ & $\Xi$ & $\stackrel{0}{r}$ & ले & $\begin{array}{l}\infty \\
\infty \\
0\end{array}$ & 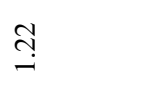 \\
\hline$\approx$ 施 & $\stackrel{q}{+}$ & $\stackrel{?}{\stackrel{0}{0}}$ & $\overrightarrow{0}$ & $\frac{5}{0}$ & $\stackrel{5}{0}$ & $\stackrel{5}{0}$ & $\stackrel{+}{0}$ & $\stackrel{\infty}{\infty}$ & $\stackrel{શ}{I}$ & $\stackrel{\wp}{\dot{\sigma}}$ & $\overrightarrow{\tilde{o}}$ & 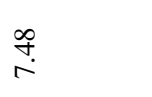 & $\stackrel{0}{0}$ & $\stackrel{0}{0}$ & $\underset{\Xi}{\overrightarrow{0}}$ \\
\hline $\bar{n}$ & $\stackrel{t}{0}$ & $\stackrel{n}{0}$ & $\stackrel{8}{\circ}$ & $\stackrel{n}{0}$ & $\stackrel{8}{\circ}$ & $\stackrel{\tilde{o}}{\circ}$ & $\stackrel{8}{0}$ & $\stackrel{\infty}{\circ}$ & $\stackrel{ \pm}{0}$ & $\stackrel{t}{0}$ & $\stackrel{t}{0}$ & $\stackrel{0}{0}$ & $\stackrel{\infty}{0}$ & $\stackrel{8}{0}$ & $\stackrel{t}{0}$ \\
\hline 㣢高 & $\stackrel{n}{\circ}$ & $\bar{n}$ & ֶֻ & : & 范 & $\tilde{m}$ & $\stackrel{0}{0}$ & $\stackrel{\circ}{~}$ & $\stackrel{m}{-}$ & $\stackrel{0}{0}$ & $\cong$ & $\hat{o}$ & $\tilde{n}$ & 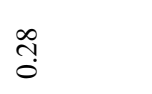 & 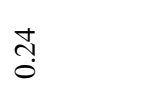 \\
\hline$\underset{G}{Q}$ & $\stackrel{\Omega}{o}$ & in & ส̃ & $\stackrel{8}{0}$ & $\stackrel{n}{n}$ & ֻै & $\stackrel{t}{0}$ & $\vec{\sigma}$ & ঙे & $\stackrel{\infty}{0}$ & $\stackrel{ \pm}{ \pm}$ & $\stackrel{\Re}{0}$ & $\vec{n}$ & $\hat{\widehat{o}}$ & $\stackrel{\overbrace{}}{0}$ \\
\hline 㕝 & 常 & 窟 & 尝 & 总 & 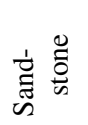 & 䆣 & 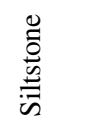 & 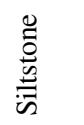 & 䆣总 & 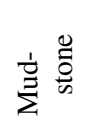 & 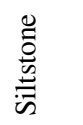 & 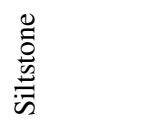 & 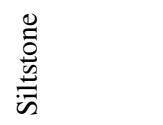 & 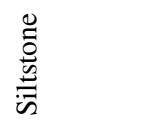 & 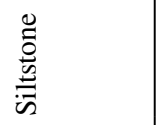 \\
\hline 芯 & 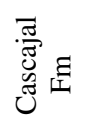 & 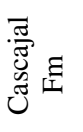 & 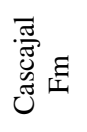 & 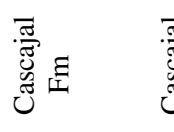 & 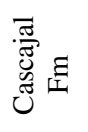 & 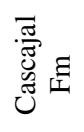 & 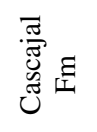 & 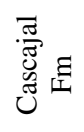 & 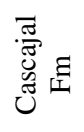 & 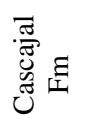 & 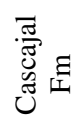 & 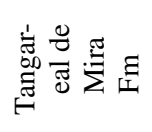 & 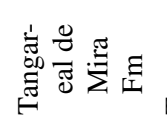 & 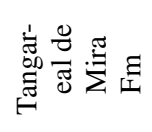 & 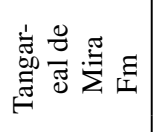 \\
\hline 吉金 & $\stackrel{\circ}{n}$ & $\stackrel{尺}{i}$ & $\stackrel{2}{2}$ & ஜ & 尽 & হু & हి & $\stackrel{9}{=}$ & $\stackrel{2}{\Xi}$ & 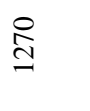 & $\stackrel{\searrow}{\beth}$ & 苞 & $\stackrel{\overbrace{}}{2}$ & 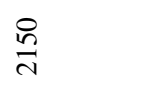 & $\stackrel{?}{\stackrel{2}{N}}$ \\
\hline 范 & $\stackrel{\substack{n \\
H}}{H}$ & 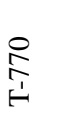 & $\underset{i}{\stackrel{9}{1}}$ & $\begin{array}{l}\stackrel{8}{\infty} \\
\infty \\
1 \\
1 \\
1\end{array}$ & 呑 & $\begin{array}{l}8 \\
\stackrel{1}{1}\end{array}$ & $\frac{8}{1}$ & $\underset{\oplus}{\stackrel{9}{\rightleftarrows}}$ & $\underset{H}{\stackrel{\rightleftharpoons}{F}}$ & $\underset{H}{\stackrel{尺}{\leftrightarrows}}$ & $\underset{H}{\stackrel{\overbrace{}}{1}}$ & $\underset{H}{\stackrel{P}{+}}$ & $\frac{\stackrel{8}{2}}{\stackrel{1}{H}}$ & $\underset{\substack{n\\
}}{\stackrel{B}{H}}$ & 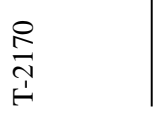 \\
\hline
\end{tabular}




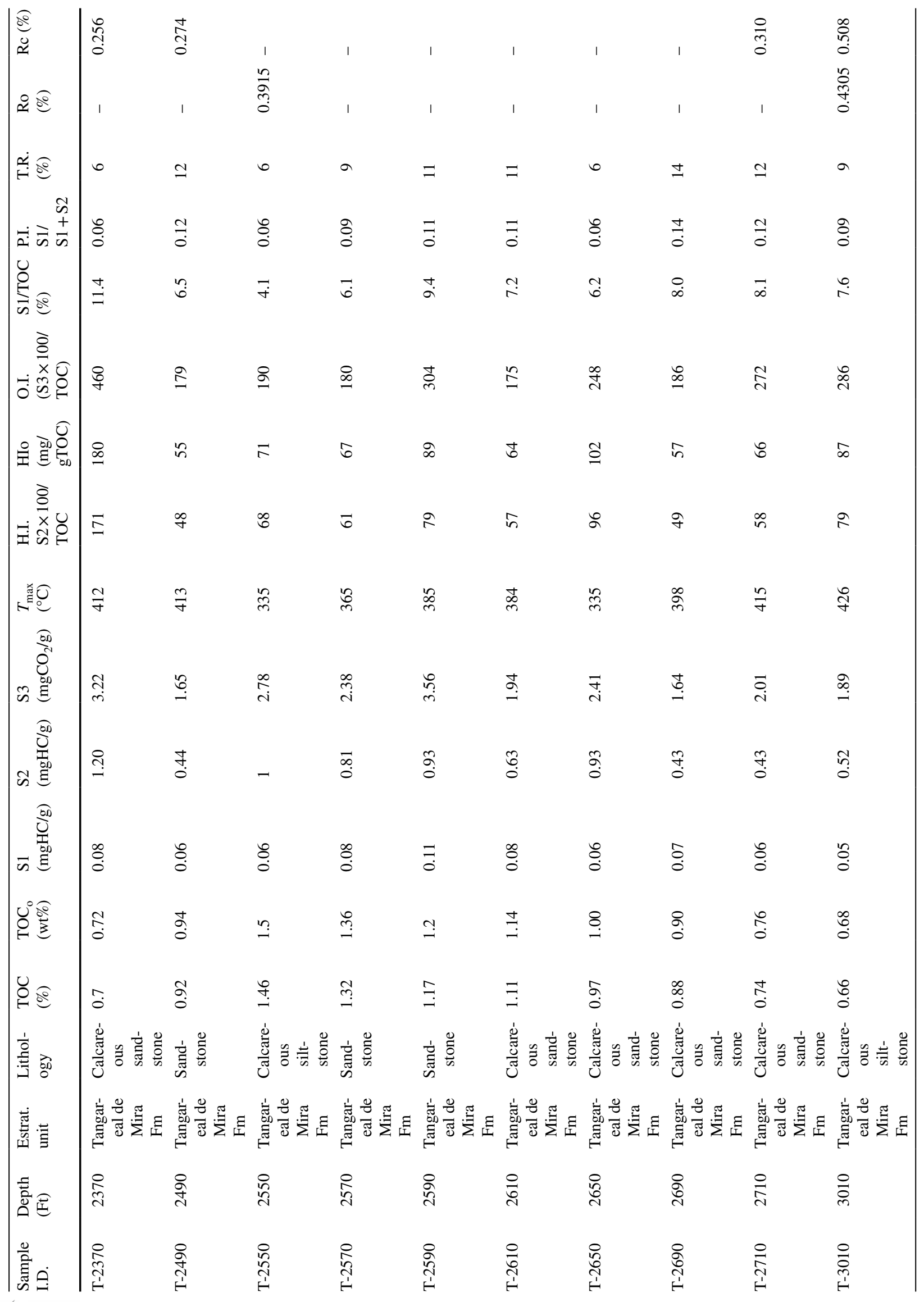




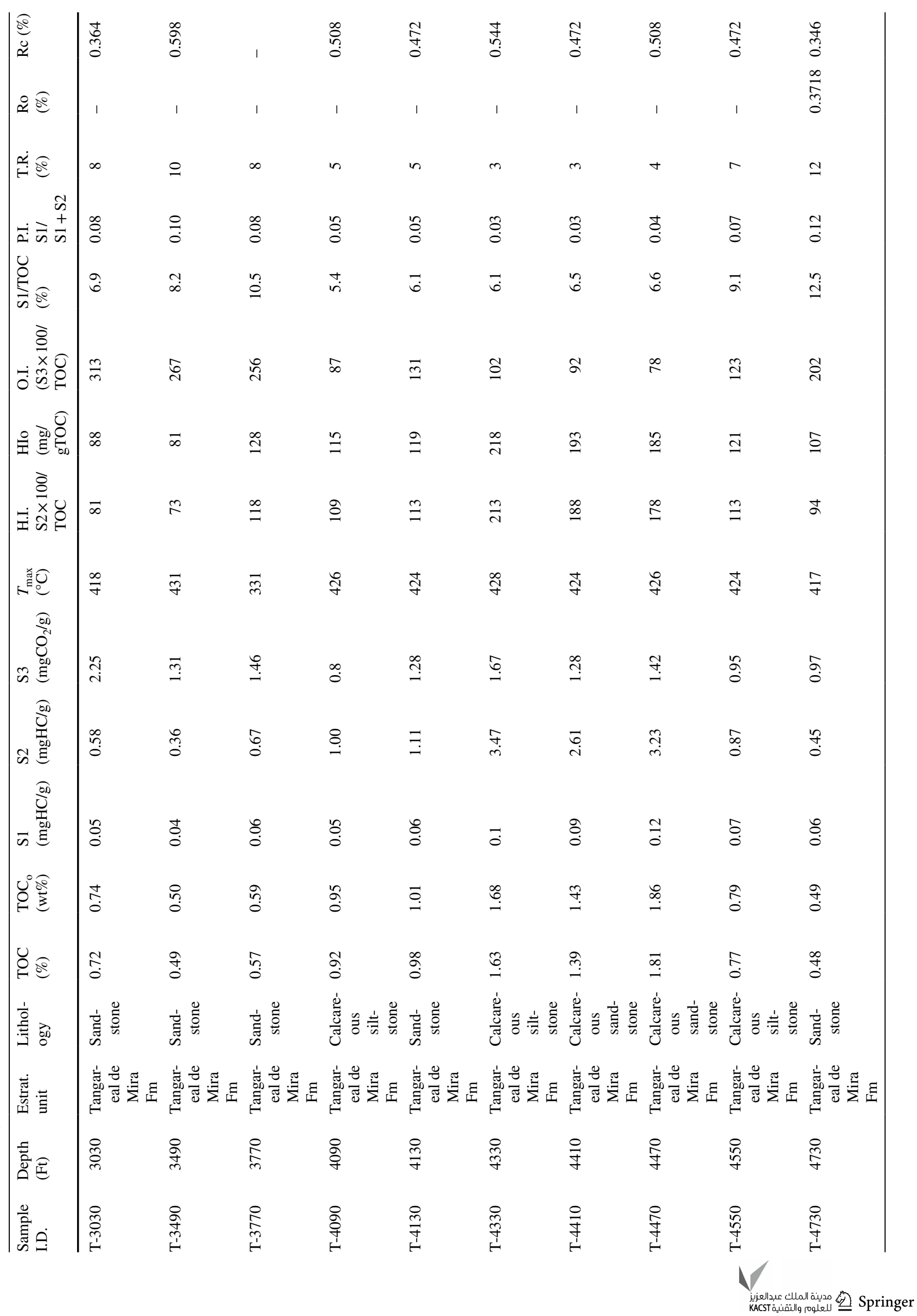




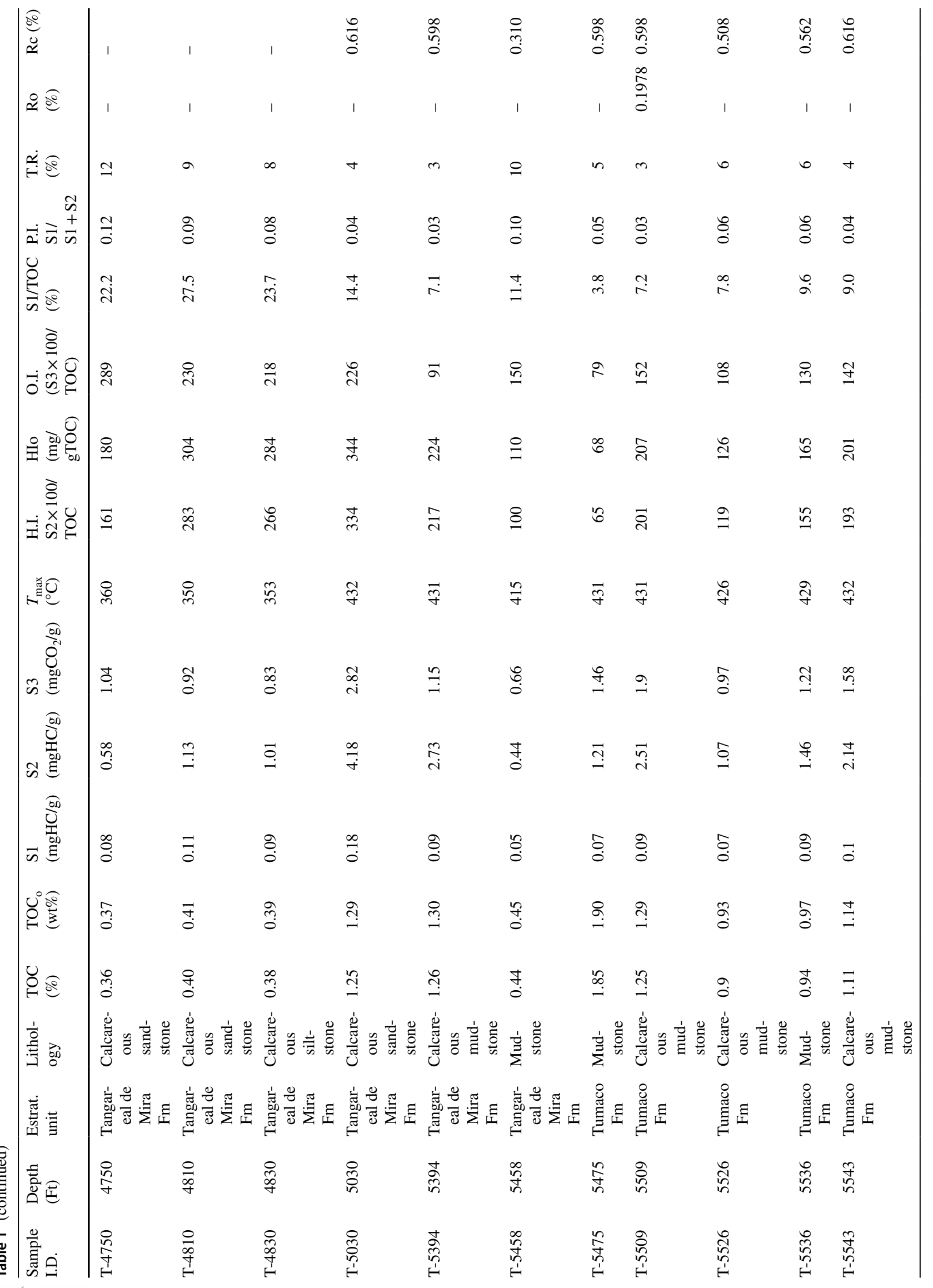




\begin{tabular}{|c|c|c|c|c|c|c|c|c|c|c|c|c|}
\hline 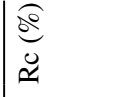 & $\begin{array}{l}\infty \\
\vdots \\
\vdots \\
0\end{array}$ & 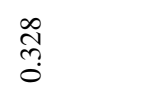 & 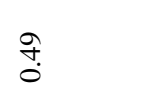 & $\stackrel{\infty}{\underset{\ddagger}{\sigma}}$ & 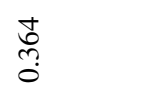 & 孛 & 导 & $\begin{array}{l}\hat{\circ} \\
\text { గి } \\
0\end{array}$ & $\begin{array}{l}\text { ते } \\
\stackrel{2}{0} \\
0\end{array}$ & $\begin{array}{l}\infty \\
\infty \\
n \\
0\end{array}$ & 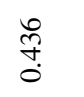 & 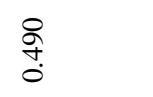 \\
\hline$\AA \widehat{\varrho}$ & 1 & $\underset{\substack{2 \\
\infty}}{0}$ & 1 & 1 & 1 & 1 & 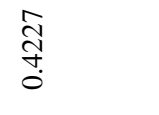 & 1 & 1 & 1 & 1 & 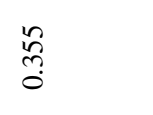 \\
\hline 象 & $n$ & 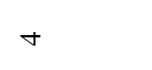 & in & N & 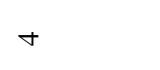 & $m$ & + & + & $m$ & N & 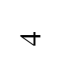 & N \\
\hline 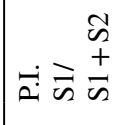 & $\stackrel{\leftrightarrow}{\circ}$ & $\stackrel{t}{\circ}$ & $\stackrel{n}{0}$ & ¿̊. & $\stackrel{t}{0}$ & $\stackrel{\leftrightarrow}{\circ}$ & Oे & $\stackrel{t}{0}$ & $\stackrel{\overbrace{}}{0}$ & ¿م. & 菅 & 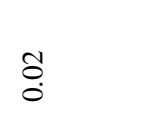 \\
\hline$\underset{0}{D}$ & 0. & $\underset{\sim}{\ddot{i}}$ & กู & $\infty_{\infty}^{m}$ & $\hat{n}$ & $\stackrel{\infty}{\sim}$ & $\stackrel{\circ}{r}$ & กู & $\ddot{n}$ & $\hat{i}$ & $\dot{i n}$ & $\underset{r}{\dot{r}}$ \\
\hline 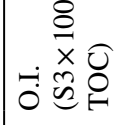 & ڤे & $i n$ & $\stackrel{\wp}{\varrho}$ & $\sigma$ & $\stackrel{2}{2}$ & 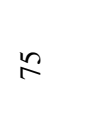 & $\hat{a}$ & $\bar{\infty}$ & $\nabla$ & $i n$ & $\bar{\Xi}$ & $\stackrel{2}{2}$ \\
\hline 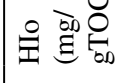 & ते & $\hat{n}$ & $\Xi$ & 导 & $\underline{\sigma}$ & $\hat{\text { ̀े }}$ & 至 & 主 & $\bar{m}$ & $\stackrel{n}{=}$ & $\stackrel{\infty}{\simeq}$ & $\stackrel{\infty}{\infty}$ \\
\hline 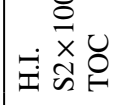 & तิ & $\tilde{n}$ & $\Xi$ & ले & $\stackrel{\text { L }}{2}$ & 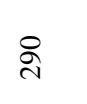 & $\stackrel{\infty}{\circ}$ & 吾 & $\widehat{\beth}$ & $\stackrel{9}{=}$ & $\tilde{\Xi}$ & $\stackrel{\infty}{\stackrel{\infty}{ }}$ \\
\hline 舀弟 & $\vec{q}$ & $\stackrel{0}{7}$ & $\stackrel{a}{q}$ & $\vec{g}$ & $\stackrel{\infty}{\forall}$ & $\stackrel{\vartheta}{\Im}$ & $\stackrel{\infty}{\sim}$ & శิ & ఫิ & $\stackrel{\overbrace{}}{\%}$ & ชิ & $\stackrel{q}{q}$ \\
\hline$\tilde{n}$ & $\stackrel{\sim}{\infty}$ & $\exists$ & $\stackrel{m}{\longrightarrow}$ & $\begin{array}{l}\infty \\
\infty \\
0\end{array}$ & $\vec{\Im}$ & $\begin{array}{l}\stackrel{0}{\infty} \\
\infty \\
0\end{array}$ & $\exists$ & $\vec{\sigma}$ & $\stackrel{\infty}{0}$ & $\begin{array}{c}\bar{\infty} \\
\dot{0}\end{array}$ & $\stackrel{\Upsilon}{ت}$ & $\stackrel{\sigma}{\sigma}$ \\
\hline$\tilde{\sigma}$ & $\stackrel{N}{i}$ & 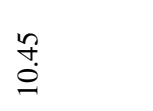 & $\stackrel{?}{-}$ & $\stackrel{\sim}{n}$ & ले & $\stackrel{m}{m}$ & 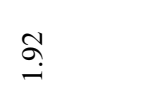 & nุ? & $\stackrel{\circ}{\stackrel{+}{*}}$ & 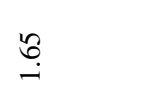 & $\stackrel{+}{\stackrel{+}{-}}$ & $\vec{n}$ \\
\hline 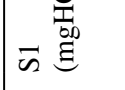 & $\overrightarrow{0}$ & f. & $\stackrel{\infty}{\circ}$ & $\stackrel{\infty}{\circ}$ & $\stackrel{8}{0}$ & $\stackrel{0}{0}$ & $\stackrel{\infty}{\circ}$ & $\stackrel{0}{\circ}$ & $\stackrel{+}{\circ}$ & $\stackrel{t}{0}$ & $\stackrel{8}{\circ}$ & $\stackrel{n}{0}$ \\
\hline $0^{0} \frac{0}{30}$ & 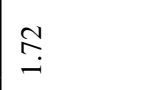 & $\stackrel{n}{a}$ & $\stackrel{m}{=}$ & gे & $\stackrel{\infty}{n}$ & $\stackrel{9}{\exists}$ & $\cong$ & $\stackrel{0}{=}$ & $\stackrel{\infty}{=}$ & $\stackrel{n}{?}$ & $\underset{ָ}{ت}$ & $\stackrel{\mathscr{P}}{-}$ \\
\hline O & $\stackrel{\leftarrow}{\circ}$ & $\stackrel{\text { aे }}{.}$ & તે & $\stackrel{\circ}{\circ}$ & $\stackrel{n}{n}$ & $\stackrel{n}{=}$ & $\stackrel{ \pm}{ت}$ & $\stackrel{m}{=}$ & $\stackrel{n}{=}$ & $\stackrel{\circ}{\stackrel{\circ}{-}}$ & $\stackrel{\ni}{\exists}$ & 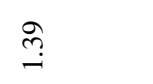 \\
\hline 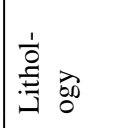 & 岂 & 岂 & 离 & 它 & 总 & 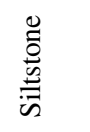 & 总 & $\frac{\mathscr{0}}{\tilde{0}}$ & 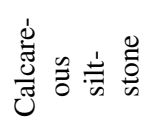 & 总 & 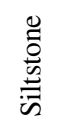 & 总 \\
\hline 鹿 & 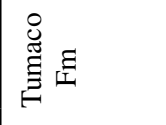 & 兽 & $\stackrel{\stackrel{8}{\mathscr{g}}}{g}$ & 兽匡 & 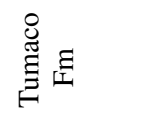 & 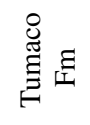 & 兽 & 总在 & 兽 & 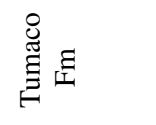 & 兽国 & 曽表 \\
\hline 咅层 & 总 & $\stackrel{8}{i n}$ & 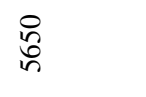 & $\underset{\substack{n \\
\text { in }}}{ }$ & 点 & $\underset{\infty}{\infty}$ & $\begin{array}{l}\stackrel{8}{\infty} \\
\stackrel{\infty}{n}\end{array}$ & 음 & 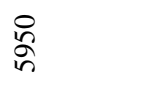 & ஓे & 웅 & ? \\
\hline ف & $\begin{array}{l}8 \\
\stackrel{0}{n} \\
n \\
1\end{array}$ & $\begin{array}{l}0 \\
n \\
n \\
n \\
n\end{array}$ & $\begin{array}{l}0 \\
6 \\
h \\
1\end{array}$ & $\underset{\substack{n \\
i \\
H}}{0}$ & $\begin{array}{l}\stackrel{8}{2} \\
n \\
n\end{array}$ & $\begin{array}{l}0 \\
\infty \\
\infty \\
1\end{array}$ & $\begin{array}{l}8 \\
\infty \\
i \\
H\end{array}$ & 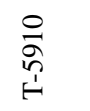 & $\begin{array}{l}8 \\
\vdots \\
n \\
n \\
1\end{array}$ & $\begin{array}{l}8 \\
\text { ลे } \\
1 \\
1\end{array}$ & $\begin{array}{l}0 \\
0 \\
0 \\
1 \\
1\end{array}$ & $\begin{array}{l}\text { P } \\
\text { O } \\
\stackrel{1}{1}\end{array}$ \\
\hline
\end{tabular}




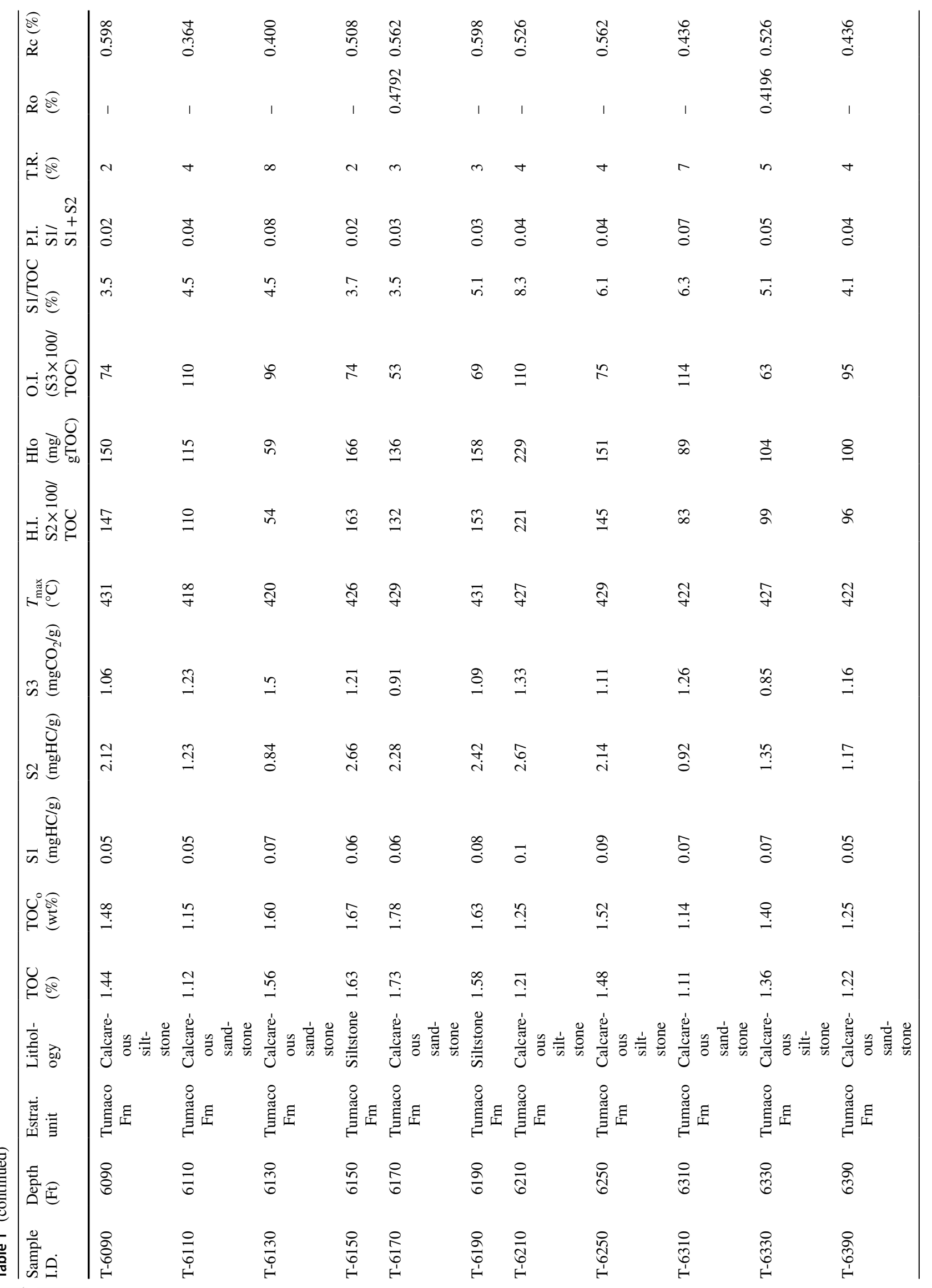




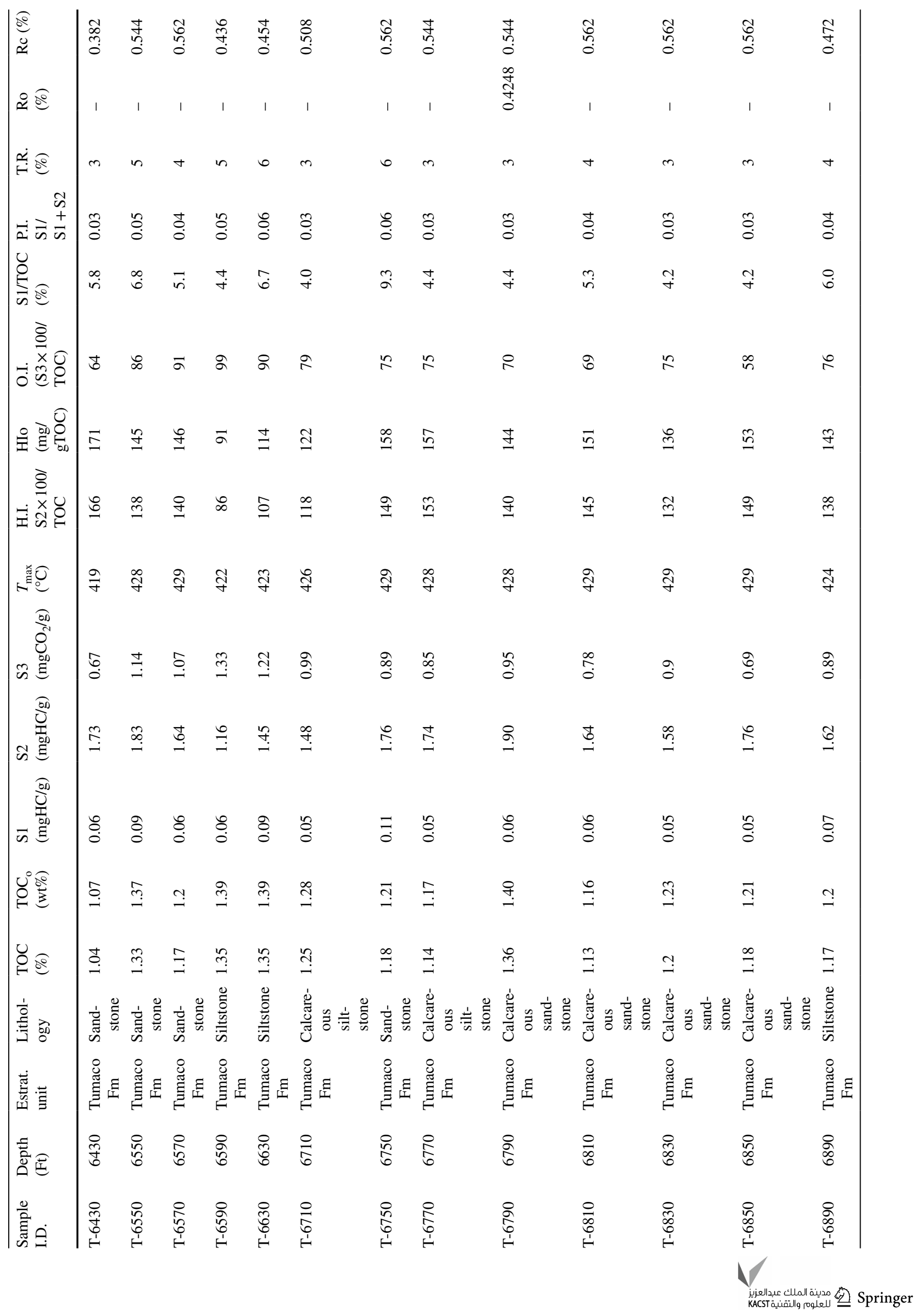




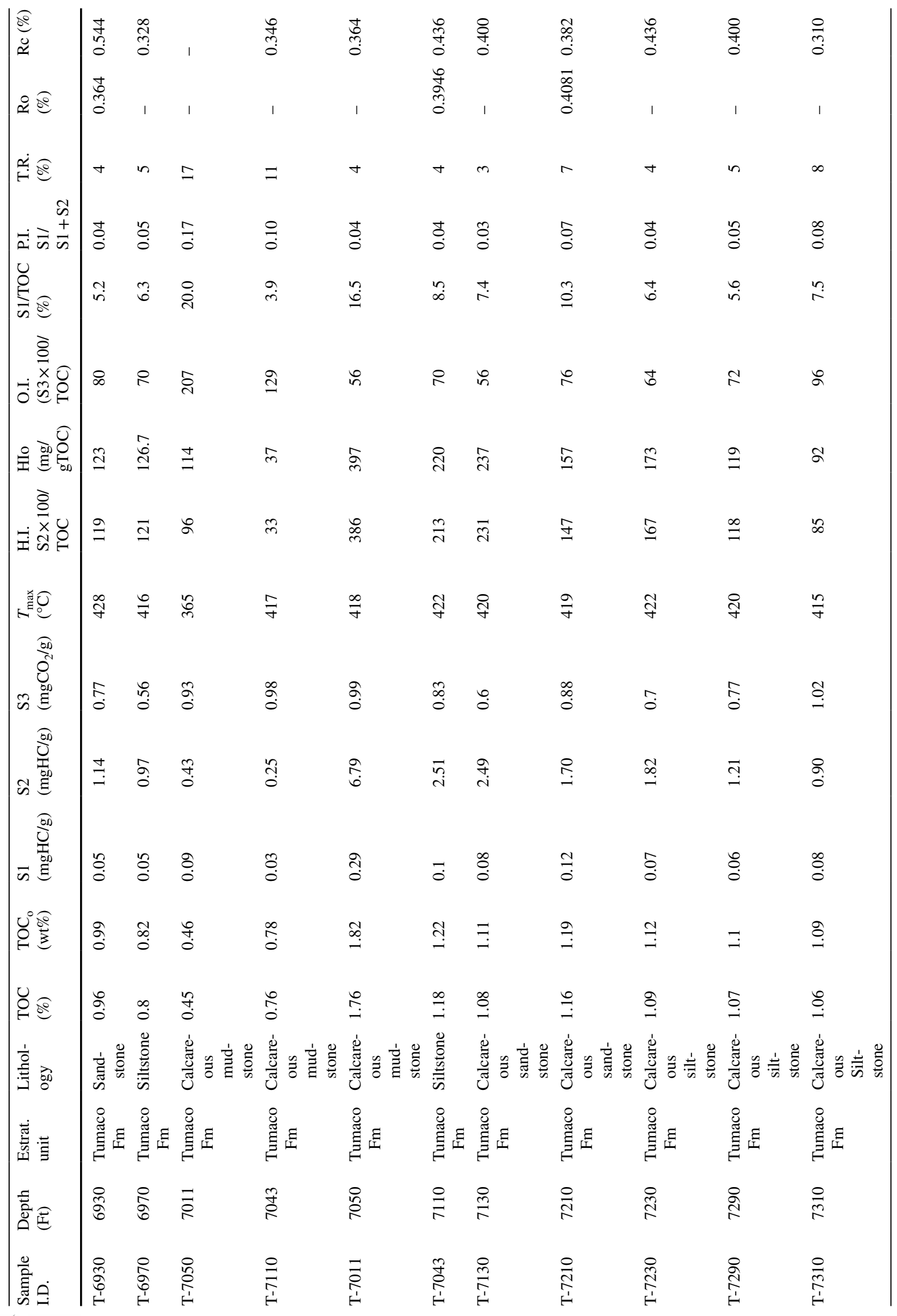




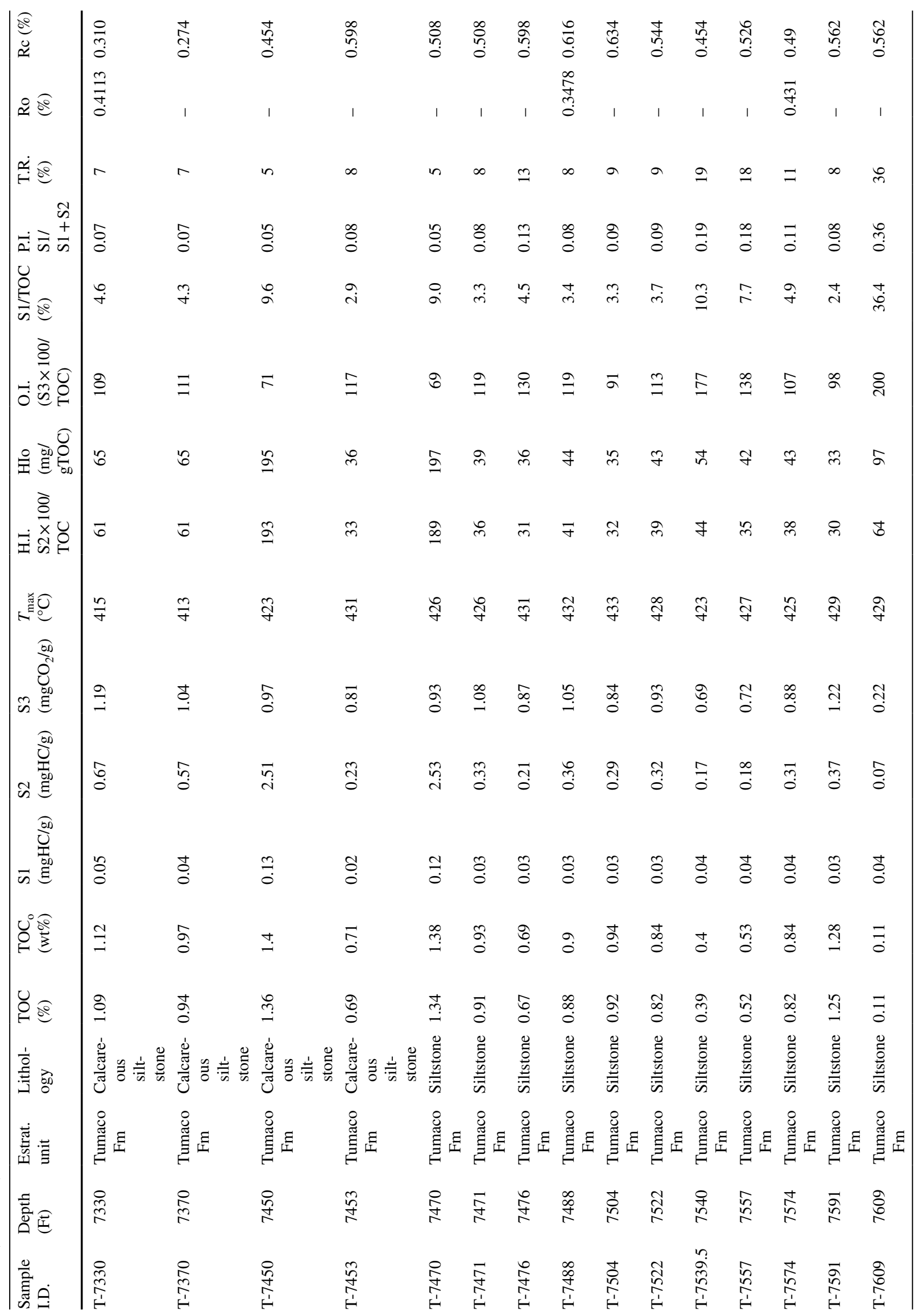




\begin{tabular}{|c|c|c|c|c|c|c|c|c|c|c|c|c|c|}
\hline $\mid \begin{array}{l}\widehat{Q} \\
\stackrel{a}{a}\end{array}$ & 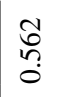 & 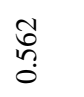 & $\stackrel{0}{0}$ & $\begin{array}{l}8 \\
0\end{array}$ & : & 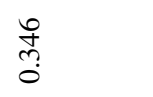 & 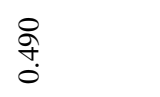 & 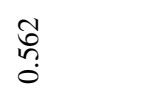 & $\stackrel{0}{0}$ & 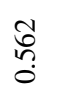 & 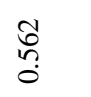 & \begin{tabular}{l}
$\infty$ \\
\multirow{2}{0}{} \\
0
\end{tabular} & 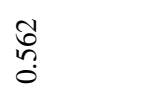 \\
\hline$\stackrel{\alpha}{a}$ & 1 & 1 & $\stackrel{\vec{n}}{\circ}$ & 1 & 1 & 苫 & 1 & $\underset{\substack{\infty \\
\infty}}{0}$ & 1 & 1 & 1 & & $\underset{\substack{\infty \\
m}}{0}$ \\
\hline$\stackrel{\leftrightarrow}{\leftrightarrow}$ & 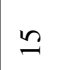 & \pm & $n$ & $\infty$ & $r$ & $\stackrel{J}{d}$ & $\stackrel{\infty}{\sim}$ & $a$ & 0 & $\cong$ & $\Xi$ & $\simeq$ & $\underline{m}$ \\
\hline 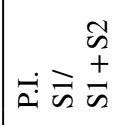 & $\stackrel{n}{0}$ & $\stackrel{\Delta}{0}$ & $\frac{n}{0}$ & $\stackrel{\infty}{0}$ & o̊. & 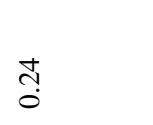 & $\stackrel{\infty}{\dddot{0}}$ & $\stackrel{8}{0}$ & $\stackrel{8}{0}$ & $\frac{m}{0}$ & $\stackrel{ \pm}{0}$ & $\stackrel{7}{0}$ & $\stackrel{9}{0}$ \\
\hline$\underset{b}{o}$ & $\overrightarrow{6}$ & $\exists$ & $\stackrel{\infty}{\dot{0}}$ & $\stackrel{\sim}{m}$ & $\stackrel{\infty}{i}$ & $\stackrel{\infty}{+}$ & $\stackrel{\infty}{a}$ & $\ddot{n}$ & $\hat{i}$ & $\stackrel{m}{+}$ & $\hat{i n}$ & $\stackrel{\vec{n}}{i}$ & $\stackrel{\infty}{\dot{m}}$ \\
\hline 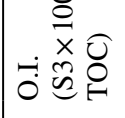 & 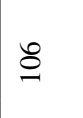 & $\vec{\nabla}$ & $\stackrel{\varrho}{\exists}$ & $\stackrel{ }{=}$ & $\stackrel{\sigma}{\varrho}$ & \& & $\stackrel{\mathscr{P}}{ \pm}$ & $\stackrel{m}{=}$ & $\stackrel{\Xi}{\circ}$ & $\stackrel{\Xi}{\varrho}$ & $\Xi$ & $\stackrel{\Re}{m}$ & $\stackrel{ \pm}{\Xi}$ \\
\hline 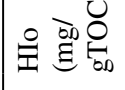 & 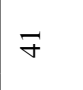 & $\stackrel{2}{2}$ & $q$ & F & $\stackrel{\infty}{m}$ & $\vec{\lambda}$ & in & $\stackrel{\infty}{m}$ & $q$ & mे & F & $\stackrel{f}{q}$ & $\vec{m}$ \\
\hline 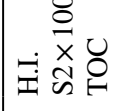 & $\ddot{m}$ & 8 & ले & के & $\tilde{m}$ & $\stackrel{0}{0}$ & f & $\ddot{m}$ & $\stackrel{f}{f}$ & $\stackrel{p}{ }$ & ల & $\vec{F}$ & $\bar{\lambda}$ \\
\hline 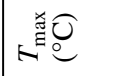 & ఫे & নे & $\stackrel{\widetilde{\gamma}}{\sigma}$ & f & $\ddot{q}$ & 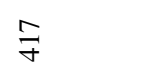 & $\stackrel{\sim}{\sigma}$ & নे & $\stackrel{\widetilde{\gamma}}{\sigma}$ & নे & নे & $\vec{g}$ & নे \\
\hline$\tilde{n}$ & $\begin{array}{l}\infty \\
\infty \\
0\end{array}$ & $\stackrel{\infty}{\infty}$ & $\tilde{0}$ & $\stackrel{m}{m}$ & $\exists$ & $\stackrel{\infty}{0}$ & $\stackrel{?}{0}$ & ஃ̊. & $\stackrel{8}{\circ}$ & ஃ̊. & ఫ̊ & $\stackrel{8}{-}$ & $\begin{array}{l}\widetilde{0} \\
0 \\
0\end{array}$ \\
\hline 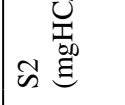 & ते & $\stackrel{n}{0}$ & $\stackrel{\overbrace{}}{0}$ & ণ্ণ & $\stackrel{\infty}{\overbrace{0}}$ & $\frac{m}{0}$ & กֶ. & @़ & $\stackrel{?}{\circ}$ & $\stackrel{\infty}{\sim}$ & $\vec{m}$ & 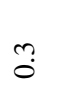 & $\overrightarrow{\tilde{\sigma}}$ \\
\hline $\bar{n}$ ह है & $\stackrel{n}{0}$ & $\stackrel{1}{0}$ & $\stackrel{0}{0}$ & $\stackrel{0}{0}$ & $\stackrel{\wp}{0}$ & $\stackrel{t}{0}$ & $\stackrel{n}{o}$ & $\stackrel{\leftrightarrow}{0}$ & $\stackrel{\leftrightarrow}{0}$ & $\stackrel{\Delta}{0}$ & $\stackrel{n}{0}$ & $\stackrel{t}{0}$ & $\stackrel{\leftrightarrow}{0}$ \\
\hline$U_{0}^{0}$ & 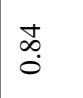 & $\hat{n}$ & $\overrightarrow{0}$ & $\stackrel{\infty}{\stackrel{\infty}{\longrightarrow}}$ & $\exists$ & $\stackrel{\wp}{\infty}$ & กี่ & $\stackrel{\infty}{\infty}$ & $\stackrel{\infty}{\circ}$ & g. & $\stackrel{\infty}{\infty}$ & $\stackrel{0}{\stackrel{0}{0}}$ & $\vec{\infty}$ \\
\hline$\underset{\wp}{\bigodot}$ & $\begin{array}{l}\stackrel{0}{0} \\
\infty \\
0\end{array}$ & 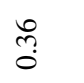 & ڤે & $\stackrel{\overbrace{}}{\overbrace{}}$ & $\stackrel{\infty}{\stackrel{\infty}{\rightarrow}}$ & $\stackrel{\infty}{\infty}$ & $\bar{n}$ & $\begin{array}{l}\infty \\
\infty \\
\infty\end{array}$ & $\stackrel{n}{\leftrightarrow}$ & ふ̆ & $\begin{array}{l}\infty \\
\infty \\
\infty\end{array}$ & $\stackrel{t}{\stackrel{0}{0}}$ & $\stackrel{\hat{\jmath}}{0}$ \\
\hline 㕝 & 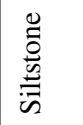 & 窵 & 窵蒿 & 总 & 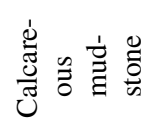 & 总 气 & 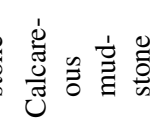 & 密 & 岂 & $\sum_{i}^{\frac{1}{2}}$ & 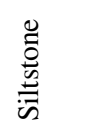 & 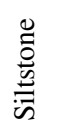 & 密 言言言 \\
\hline 焉 & 兽 & 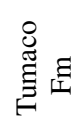 & $\stackrel{8}{\stackrel{8}{g}}$ & $\underset{\mathscr{g}}{\stackrel{g}{g}}$ & $\underset{\mathscr{g}}{\stackrel{\Xi}{g}}$ & $\stackrel{8}{\stackrel{\mathscr{J}}{g}}$ & $\stackrel{\mathscr{\Xi}}{\Xi} \Xi$ & 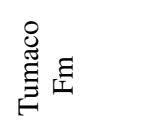 & 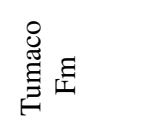 & 兽 & $\stackrel{8}{\mathscr{E}}$ & 兽 & $\stackrel{8}{\mathscr{E}}$ \\
\hline 劳尾 & $\widehat{\widehat{\sigma}}$ & fơ & ڤ్రి & శి & $\underset{⿱}{\stackrel{N}{ }}$ & 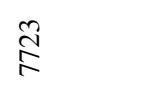 & $\underset{⿱}{\stackrel{9}{ }}$ & $\underset{\nabla}{\stackrel{0}{~}}$ & $\stackrel{尺}{E}$ & $\stackrel{n}{\curvearrowright}$ & $\underset{\substack{\sim \\
\infty}}{\stackrel{\infty}{\triangleright}}$ & $\underset{\substack{f \\
\infty}}{\mathbb{8}}$ & $\underset{\infty}{\infty}$ \\
\hline ف & 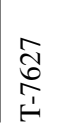 & 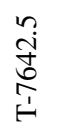 & 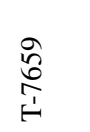 & 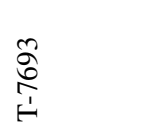 & $\underset{\stackrel{H}{H}}{\stackrel{N}{*}}$ & 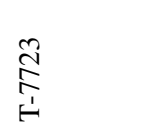 & $\underset{H}{\stackrel{9}{t}}$ & 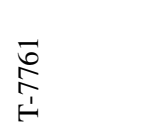 & 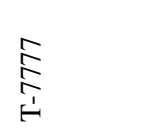 & 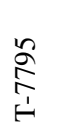 & 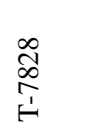 & $\underset{\substack{J \\
\stackrel{⿱}{+}}}{\stackrel{f}{H}}$ & 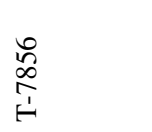 \\
\hline
\end{tabular}




\begin{tabular}{|c|c|c|c|c|c|c|c|c|c|c|c|c|c|c|}
\hline $\begin{array}{l}a \\
\stackrel{a}{a} \\
\approx\end{array}$ & مै & $\begin{array}{l}\infty \\
0 \\
0 \\
0\end{array}$ & 苞 & ڤ̊. & : & 苞 & : & $\stackrel{0}{0}$ & $\stackrel{\infty}{\stackrel{\infty}{\stackrel{0}{0}}}$ & $\stackrel{0}{0}$ & ర్t & $\frac{8}{8}$ & 离 & $\begin{array}{l}\infty \\
0 \\
0 \\
0\end{array}$ \\
\hline 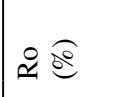 & 1 & 1 & 1 & 1 & 总 & 1 & 1 & 1 & 1 & $\stackrel{\vec{y}}{\stackrel{0}{0}}$ & 1 & 1 & 1 & 1 \\
\hline 递 & $\infty$ & 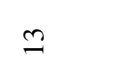 & $a$ & $r$ & in & $r$ & $n$ & $a$ & 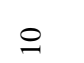 & $r$ & $n$ & $a$ & $\cong$ & $r$ \\
\hline a & $\stackrel{\infty}{0}$ & $\stackrel{m}{0}$ & oे & $\stackrel{5}{0}$ & $\stackrel{n}{0}$ & o. & $\stackrel{n}{0}$ & oे. & $\stackrel{0}{\circ}$ & $\stackrel{0}{0}$ & $\stackrel{n}{0}$ & ชุ & $\stackrel{n}{0}$ & $\stackrel{5}{\circ}$ \\
\hline 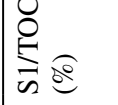 & $\hat{i}$ & $\stackrel{\sim}{+}$ & $\stackrel{0}{i}$ & $\stackrel{i}{i}$ & 9 & $\stackrel{\vec{r}}{r}$ & $\stackrel{\infty}{-}$ & $\stackrel{\sim}{m}$ & $\stackrel{\infty}{+}$ & $\stackrel{m}{m}$ & $\stackrel{9}{-}$ & $\overline{\vec{j}}$ & $\vec{a}$ & $\hat{i}$ \\
\hline $\begin{array}{rl} & \bar{x} \\
-i & \tilde{E} \\
0 & 0\end{array}$ & $\infty$ & $\stackrel{\imath}{\varrho}$ & $\stackrel{\vartheta}{\varrho}$ & $\underline{0}$ & 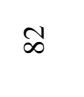 & 3 & $\infty$ & ఠิ & 2 & $\triangleright$ & $\stackrel{2}{1}$ & 苔 & $\stackrel{\text { ஜ }}{2}$ & $F$ \\
\hline 急密 & $\stackrel{p}{n}$ & లె & $\bar{m}$ & $\hat{\imath}$ & $\bar{\gamma}$ & + & $\stackrel{\infty}{\infty}$ & $\hat{m}$ & r & $q$ & $\underset{m}{\infty}$ & 8 & $\stackrel{\infty}{n}$ & $\stackrel{\infty}{m}$ \\
\hline 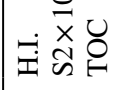 & $m$ & ते & $\stackrel{\infty}{\sim}$ & $\ddot{c}$ & ले & $\mathscr{F}$ & $\stackrel{n}{n}$ & $\stackrel{+}{m}$ & $\vec{\gamma}$ & $q$ & $\ddot{n}$ & 8 & in & $\ddot{m}$ \\
\hline N & $\vec{F}$ & $\stackrel{\mathscr{F}}{\mathscr{f}}$ & $\mathscr{\gamma}$ & $\vec{q}$ & $\stackrel{n}{q}$ & $\stackrel{\Re}{\vartheta}$ & $\ddot{\gamma}$ & $\stackrel{\widetilde{\sigma}}{ซ}$ & $\vec{f}$ & $\stackrel{\widetilde{f}}{f}$ & $\stackrel{m}{q}$ & \& & 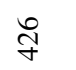 & $\underset{\wp}{\mathscr{q}}$ \\
\hline$\tilde{n}$ & $\stackrel{2}{\circ}$ & $\stackrel{\vec{\sigma}}{-}$ & n & $\vec{i}$ & $\stackrel{I}{I}$ & $n$ & $\stackrel{\infty}{\infty}$ & $\dddot{\sigma}$ & n̊. & $\stackrel{t}{\stackrel{0}{0}}$ & $\begin{array}{l}0 \\
0 \\
0\end{array}$ & ֻุ & $\stackrel{m}{0}$ & $\stackrel{\imath}{0}$ \\
\hline$\approx \stackrel{\substack{0 \\
\frac{T}{60}}}{g}$ & $\hat{n}$ & 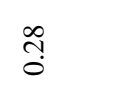 & $\stackrel{\text { fo }}{0}$ & n̊. & $\begin{array}{l}\text { Co } \\
\stackrel{0}{0}\end{array}$ & $\stackrel{\infty}{0}$ & $\stackrel{\infty}{\stackrel{\infty}{0}}$ & ڤై & ֶֻ. & $\stackrel{t}{0}$ & $\stackrel{n}{0}$ & $\stackrel{8}{0}$ & $\exists$ & के \\
\hline $\bar{n}$ 兑 & $\stackrel{0}{0}$ & $\begin{array}{l}0 \\
0 \\
0\end{array}$ & 苞 & 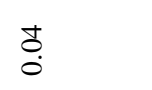 & $\stackrel{t}{0}$ & $\stackrel{0}{0}$ & $\stackrel{t}{0}$ & $\stackrel{\leftrightarrow}{0}$ & $\stackrel{\leftrightarrow}{0}$ & $\stackrel{8}{0}$ & రి & $\stackrel{\leftrightarrow}{0}$ & 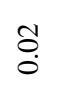 & $\stackrel{\tilde{o}}{0}$ \\
\hline$\underbrace{0}_{0} \frac{8}{8}$ & \pm & $\grave{o}$ & ñ & $N$ & $\stackrel{0}{i}$ & $\dot{o}$ & 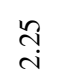 & $\stackrel{n}{o}$ & $\stackrel{n}{0}$ & $\stackrel{+}{\circ}$ & $\stackrel{0}{\circ}$ & $\frac{m}{0}$ & $\stackrel{\overbrace{}}{0}$ & $\stackrel{n}{=}$ \\
\hline$\underset{\theta}{0}$ & $\exists$ & ஜ̊ & $\stackrel{n}{n}$ & $\stackrel{\circ}{-}$ & $\vec{i}$ & 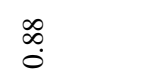 & $\stackrel{\vec{\lambda}}{i}$ & $\stackrel{\tilde{o}}{0}$ & $\stackrel{8}{0}$ & $\hat{\sigma}$ & $\stackrel{\Xi}{\text { ț }}$ & $\stackrel{m}{0}$ & สุ & $\cong$ \\
\hline 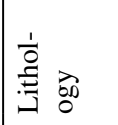 & 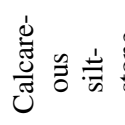 & 怤 & 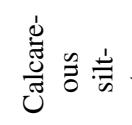 & 总 & 窟 & 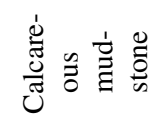 & 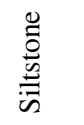 & 密 & 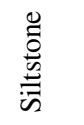 & 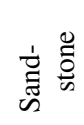 & 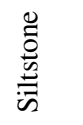 & 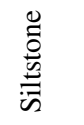 & 亲 & 总 \\
\hline 焉 & 兽 & 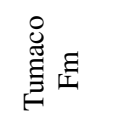 & 兽国 & 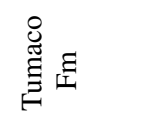 & 兽 & 兽 & 总 & 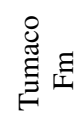 & 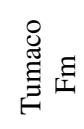 & 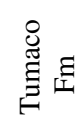 & $\stackrel{8}{\mathscr{E}}$ & 兽目 & 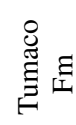 & 兽死 \\
\hline 咅 & $\underset{\triangleright}{\triangleright}$ & $\underset{\infty}{\stackrel{\infty}{\infty}}$ & $\stackrel{n}{\propto}$ & $\overline{2}$ & $\stackrel{\infty}{\stackrel{2}{\Sigma}}$ & $\stackrel{n}{2}$ & $\stackrel{\overrightarrow{2}}{2}$ & $\stackrel{\vec{\infty}}{\vec{D}}$ & $\underset{\infty}{\stackrel{2}{0}}$ & 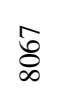 & $\stackrel{+}{+}$ & $\frac{n}{\stackrel{n}{0}}$ & $\underset{\infty}{\tilde{j}}$ & $\frac{\mathfrak{q}}{\infty}$ \\
\hline ف를 & $\begin{array}{l}\overrightarrow{0} \\
\stackrel{0}{1} \\
1\end{array}$ & $\begin{array}{l}\infty \\
\infty \\
\infty \\
1\end{array}$ & 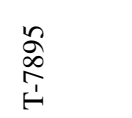 & $\overrightarrow{\bar{\sigma}}$ & 岕 & $\begin{array}{l}n \\
\hat{\alpha} \\
1\end{array}$ & $\stackrel{\vec{a}}{\stackrel{1}{1}}$ & 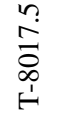 & $\begin{array}{l}\text { vo } \\
\text { o } \\
\infty \\
i\end{array}$ & $\begin{array}{l}\hat{8} \\
\infty \\
1 \\
1\end{array}$ & $\begin{array}{l}\begin{array}{l}+ \\
\infty \\
\infty \\
\infty \\
i\end{array} \\
H\end{array}$ & 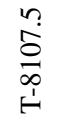 & 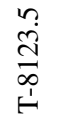 & $\begin{array}{l}\frac{n}{f} \\
\substack{0 \\
H}\end{array}$ \\
\hline
\end{tabular}




\begin{tabular}{|c|c|c|c|c|c|c|c|c|c|c|c|c|c|}
\hline $\mid \begin{array}{l}\widehat{Q} \\
\stackrel{a}{a}\end{array}$ & $\begin{array}{l}\infty \\
\vdots \\
0 \\
0\end{array}$ & $\begin{array}{l}\infty \\
\stackrel{2}{0} \\
\stackrel{0}{0}\end{array}$ & $\stackrel{0}{0}$ & 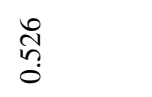 & 苞 & 苞 & $\frac{\mathfrak{T}}{\stackrel{0}{0}}$ & 苞 & $\stackrel{0}{0}$ & 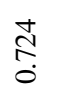 & బ్ & $\stackrel{8}{\stackrel{0}{0}}$ & 苞 \\
\hline$\approx \widehat{Q}$ & $\begin{array}{l}\mathscr{N} \\
\stackrel{0}{+} \\
\stackrel{0}{0}\end{array}$ & 1 & 1 & 1 & 1 & $\stackrel{\infty}{\infty}$ & 1 & 1 & & ڤ్ & 1 & 1 & 1 \\
\hline 总 & 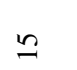 & $\infty$ & 0 & $\infty$ & $r$ & in & 0 & $r$ & 0 & 0 & 0 & in & 으 \\
\hline 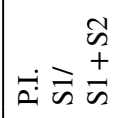 & $\frac{n}{0}$ & $\stackrel{\infty}{\circ}$ & $\stackrel{8}{0}$ & $\stackrel{\infty}{\circ}$ & $\stackrel{0}{0}$ & $\stackrel{n}{0}$ & $\stackrel{8}{0}$ & $\stackrel{0}{0}$ & $\stackrel{8}{0}$ & $\stackrel{0}{0}$ & $\stackrel{8}{0}$ & $\stackrel{8}{0}$ & $\frac{0}{0}$ \\
\hline$\underset{b}{o}$ & $\hat{\circ}$ & $\hat{i}$ & $\stackrel{m}{i}$ & $\vec{i}$ & $\ddot{n}$ & $\stackrel{\circ}{-}$ & $\ddot{n}$ & $\stackrel{\circ}{i}$ & $\vec{m}$ & $\stackrel{+}{\oplus}$ & $\stackrel{+}{\oplus}$ & $\underset{i}{0}$ & $\stackrel{\sim}{+}$ \\
\hline 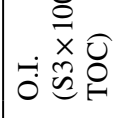 & $\stackrel{\varrho}{=}$ & $\stackrel{\infty}{\circ}$ & $\Re$ & $\stackrel{m}{=}$ & $\stackrel{\curvearrowleft}{=}$ & $\kappa$ & తి & $\stackrel{\overbrace{}}{\varrho}$ & $\bar{a}$ & 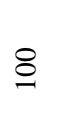 & $\infty$ & $\kappa$ & $\stackrel{m}{\exists}$ \\
\hline 点 & $\nabla$ & ల్ల & $\stackrel{\infty}{\infty}$ & $\hat{\lambda}$ & ల & ఠ్ల & $\bar{n}$ & $\stackrel{\infty}{\sim}$ & t & $n$ & $n$ & $\stackrel{\infty}{+}$ & \& \\
\hline 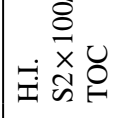 & $\vec{b}$ & $m$ & ల & $\ddot{\sim}$ & 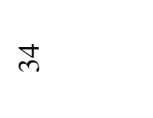 & নे & $\stackrel{\infty}{+}$ & $\stackrel{\sim}{\sim}$ & 8 & in & in & f & i \\
\hline 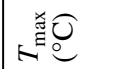 & $\vec{q}$ & $\bar{\Im}$ & $\tilde{ซ}$ & $\widehat{\mathscr{F}}$ & $\ddot{\vartheta}$ & $\ddot{\vartheta}$ & ફे & $\ddot{r}$ & $\stackrel{\sim}{q}$ & $\stackrel{\infty}{\underset{\gamma}{\sigma}}$ & $\stackrel{+}{\stackrel{F}{\sigma}}$ & $\hat{q}$ & $\stackrel{m}{q}$ \\
\hline$\approx \stackrel{0.0}{\Xi}$ & $\stackrel{\infty}{0}$ & $\stackrel{8}{-}$ & $\stackrel{n}{o}$ & $\stackrel{n}{6}$ & $\stackrel{\tilde{m}}{=}$ & $\stackrel{n}{\stackrel{n}{i}}$ & $\stackrel{m}{=}$ & $\stackrel{\circ}{i}$ & $\stackrel{+}{]}$ & $\stackrel{0}{=}$ & gे & $\stackrel{\overbrace{}}{\stackrel{f}{-}}$ & 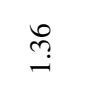 \\
\hline$\tilde{\sigma}$ & $\frac{5}{0}$ & ले & f̊ & ?ִ & $\stackrel{\leftrightarrow}{0}$ & ț. & ?̊ & 䓹 & $\begin{array}{l}\stackrel{1}{0} \\
0 \\
0\end{array}$ & $\stackrel{\infty}{n}$ & nุ? & $\vec{i}$ & $\stackrel{\text { fo }}{0}$ \\
\hline $\bar{n}$ 莡 & $\stackrel{\leftrightarrow}{0}$ & $\stackrel{8}{0}$ & $\stackrel{8}{0}$ & $\stackrel{8}{0}$ & $\stackrel{\wp}{0}$ & $\stackrel{\ddot{c}}{0}$ & $\stackrel{\wp}{0}$ & $\stackrel{t}{0}$ & $\stackrel{n}{0}$ & $\stackrel{0}{0}$ & $\stackrel{t}{0}$ & $\stackrel{t}{0}$ & $\stackrel{2}{0}$ \\
\hline 婂递 & ते & $\stackrel{n}{\ddot{z}}$ & $\stackrel{m}{=}$ & $\because$ & $\cong$ & $\stackrel{t}{g}$ & ڤ̊ & $\vec{i}$ & $\stackrel{+}{-}$ & $\stackrel{9}{=}$ & 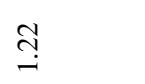 & in & $\stackrel{\overbrace{}}{\overbrace{}}$ \\
\hline Oe & 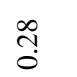 & $\underset{\sigma}{\sigma}$ & $\stackrel{m}{-}$ & $\stackrel{?}{\stackrel{f}{i}}$ & $\stackrel{9}{\leftrightarrows}$ & $\stackrel{\infty}{\rightarrow}$ & $\stackrel{+}{\circ}$ & $\underset{i}{\tilde{i}}$ & $\stackrel{\mathscr{n}}{\longrightarrow}$ & $\stackrel{0}{\longrightarrow}$ & $\stackrel{\jmath}{\leftrightarrows}$ & $\stackrel{n}{n}$ & 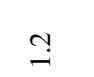 \\
\hline 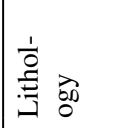 & 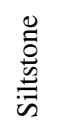 & 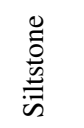 & $\sum_{i}^{\frac{1}{2}}$ & 总 气 & 总 & 矛 & 密 & 总 & 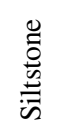 & 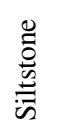 & 造 & $\frac{\mathscr{0}}{0.0}$ & 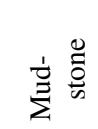 \\
\hline 崖 & 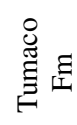 & 兽 & 总表 & 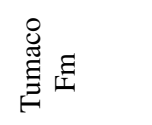 & 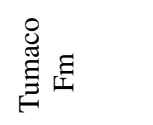 & 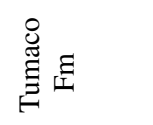 & 兽看 & 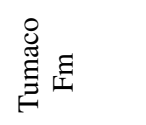 & 兽 & 兽 & 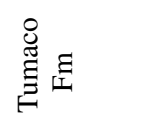 & 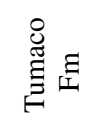 & 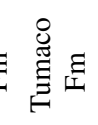 \\
\hline 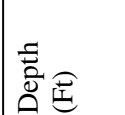 & $\begin{array}{l}n \\
0 \\
\infty \\
\infty\end{array}$ & $\frac{\dot{\sigma}}{\infty}$ & $\underset{\infty}{\overline{1}}$ & 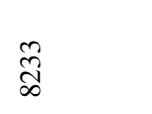 & $\underset{\infty}{\stackrel{్}{d}}$ & $\begin{array}{l}n \\
n \\
n \\
\infty\end{array}$ & $\begin{array}{l}\text { ర్ర } \\
\infty\end{array}$ & 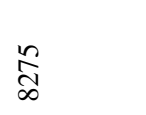 & 虽 & $\frac{\mathfrak{n}}{\stackrel{n}{\infty}}$ & 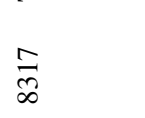 & $\begin{array}{l}n \\
i \\
\infty \\
\infty\end{array}$ & $\aleph_{\infty}^{\infty}$ \\
\hline 䓂. & $\begin{array}{l}n \\
0 \\
0 \\
\infty \\
1 \\
1\end{array}$ & 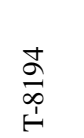 & $\begin{array}{l}\vec{\pi} \\
\substack{1 \\
i \\
i}\end{array}$ & $\begin{array}{l}\tilde{N} \\
\infty \\
\stackrel{H}{H}\end{array}$ & $\underset{\substack{\mathcal{H} \\
\infty \\
\stackrel{M}{H}}}{ }$ & $\begin{array}{l}n \\
n \\
n \\
\infty \\
\infty \\
1\end{array}$ & 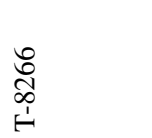 & 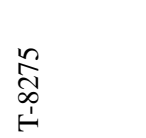 & 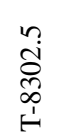 & 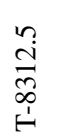 & 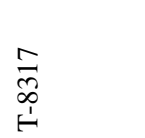 & $\begin{array}{l}\tilde{n} \\
\tilde{n} \\
\infty \\
\infty \\
i \\
i\end{array}$ & ל. \\
\hline
\end{tabular}




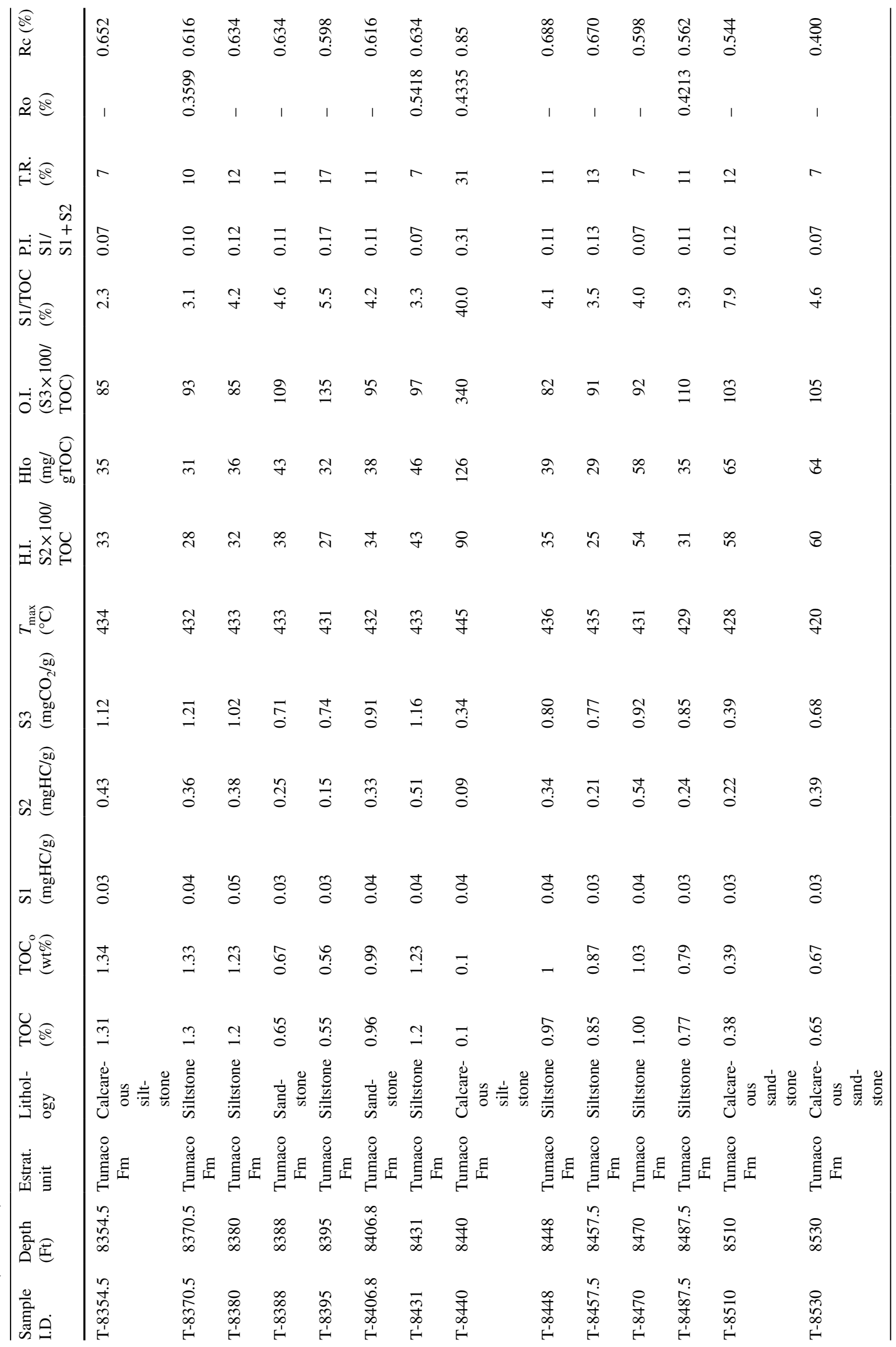




\begin{tabular}{|c|c|c|c|c|c|c|c|c|c|c|c|}
\hline 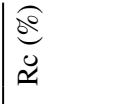 & $\frac{0}{3}$ & $\stackrel{\circ}{m}$ & $\stackrel{0}{m}$ & 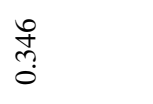 & 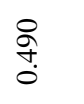 & $\begin{array}{l}\infty \\
\text { nิ } \\
0\end{array}$ & $\begin{array}{l}0 \\
0 \\
0 \\
0\end{array}$ & $\stackrel{\infty}{n}$ & $\begin{array}{l}\text { त्र } \\
\text { ?ֶ. }\end{array}$ & $\begin{array}{l}\text { त्र } \\
\text { ?ֶ. }\end{array}$ & $\begin{array}{l}\text { त्र } \\
\stackrel{n}{0}\end{array}$ \\
\hline$\approx \cong$ & 1 & 1 & 1 & 1 & 1 & 1 & 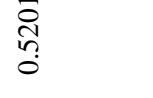 & 1 & 1 & 1 & 1 \\
\hline نج & \pm & $=$ & $=$ & $=$ & $=$ & + & $r$ & 0 & $r$ & $r$ & 0 \\
\hline 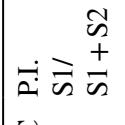 & $\stackrel{ \pm}{0}$ & $=$ & $\overline{0}$ & $=$ & $=$ & d. & : & $\stackrel{8}{\circ}$ & $\stackrel{5}{\circ}$ & : & $\stackrel{8}{\circ}$ \\
\hline$\stackrel{8}{=}$ & 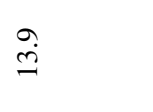 & 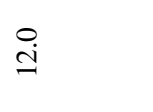 & $n$ & $\stackrel{m}{-}$ & $\stackrel{\circ}{\circ}$ & $\stackrel{\vec{i}}{i}$ & $\stackrel{\vec{r}}{\dot{m}}$ & $\stackrel{\infty}{\sim}$ & $\stackrel{\leftrightarrow}{\dot{f}}$ & $n$ & $\stackrel{\sim}{+}$ \\
\hline 齐式 & สี & I & $\stackrel{\wp}{n}$ & $\Xi$ & 8 & $\infty$ & $\infty$ & $\infty$ & $\stackrel{\infty}{\simeq}$ & $\widehat{\beth}$ & ă \\
\hline 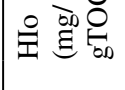 & ฉิ & $\hat{\sigma}$ & $\infty$ & $\stackrel{-}{\circ}$ & $\infty$ & $\overrightarrow{6}$ & $\tilde{n}$ & t & $\approx$ & d & $\infty$ \\
\hline 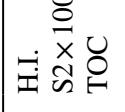 & $\infty$ & ๙ & 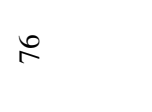 & $\stackrel{\infty}{\circ}$ & $\stackrel{+}{\sim}$ & in & $\stackrel{g}{\sigma}$ & 8 & 5 & $\infty$ & t \\
\hline 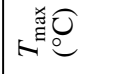 & $\stackrel{n}{\forall}$ & $\frac{n}{\gamma}$ & $\stackrel{n}{\forall}$ & $\stackrel{\ni}{\ni}$ & 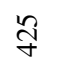 & $\vec{\vartheta}$ & $\stackrel{\widetilde{\sigma}}{\sim}$ & $\vec{\vartheta}$ & ఫे & ণे & ঙิ \\
\hline$\tilde{n}$ & $\begin{array}{l}\infty \\
0 \\
0\end{array}$ & $\stackrel{\overrightarrow{0}}{0}$ & $\stackrel{0}{0}$ & $\stackrel{n}{o}$ & $\stackrel{\infty}{\stackrel{0}{0}}$ & ڤे & $\stackrel{\vartheta}{\ominus}$ & $\underset{-}{F}$ & $\stackrel{t}{\rightarrow}$ & $\stackrel{\infty}{\circ}$ & $\exists$ \\
\hline 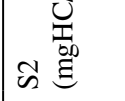 & $\vec{m}$ & $\stackrel{\infty}{0}$ & กี & $\stackrel{\overbrace{}}{\hat{\sigma}}$ & $\stackrel{\infty}{n}$ & $\stackrel{?}{\stackrel{0}{0}}$ & - & $\hat{o}$ & $\stackrel{+}{a}$ & $\stackrel{\infty}{\circ}$ & $\stackrel{尺}{\stackrel{0}{0}}$ \\
\hline $\bar{n} \stackrel{\frac{T}{60}}{g}$ & $\stackrel{n}{0}$ & $\stackrel{8}{\circ}$ & $\stackrel{d}{0}$ & $\overrightarrow{0}$ & $\stackrel{0}{0}$ & $\stackrel{\wp}{0}$ & $\stackrel{0}{\circ}$ & $\stackrel{8}{\circ}$ & $\stackrel{t}{0}$ & $\overbrace{0}^{2}$ & $\stackrel{2}{0}$ \\
\hline 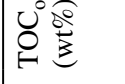 & ڤ̊. & $\vec{n}$ & $\stackrel{?}{\stackrel{0}{0}}$ & $\stackrel{\infty}{\infty}$ & $\stackrel{\infty}{0}$ & 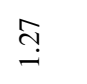 & $\underset{\text { i }}{\text { i }}$ & $\stackrel{\text { SO}}{-}$ & $\begin{array}{l}\infty \\
\infty \\
0\end{array}$ & $\stackrel{2}{0}$ & $\stackrel{\overbrace{}}{\longrightarrow}$ \\
\hline$\underset{\theta}{0}$ & ֻి & ֻ̊ & $\stackrel{\text { I }}{\circ}$ & $\vec{\infty}$ & $\stackrel{\infty}{\stackrel{0}{0}}$ & $\stackrel{+}{\rightarrow}$ & $\underset{i}{\stackrel{t}{i}}$ & $\stackrel{\infty}{n}$ & $\begin{array}{l}\vec{\infty} \\
\dot{0}\end{array}$ & $\stackrel{\hat{\imath}}{0}$ & $\stackrel{\overbrace{}}{-}$ \\
\hline 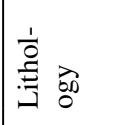 & 总 & 总 & 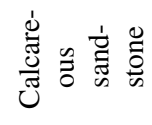 & 总 & 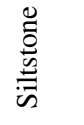 & 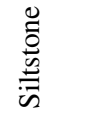 & 总 气 离 总 & 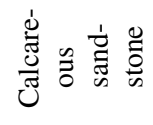 & 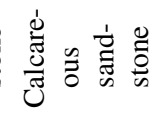 & 总 & 总 \\
\hline 焉 & 兽 & 兽 & 总囯 & 兽 & 总国 & 总死 & 总 & 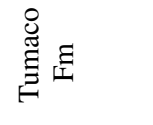 & 莺 & 兽 & 兽国 \\
\hline 咅国 & $\begin{array}{l}\not \\
\infty \\
\infty\end{array}$ & ஃ্ণ & ڤ్రి & $\underset{\infty}{\stackrel{2}{\infty}}$ & $\underset{\infty}{\stackrel{2}{\infty}}$ & $\begin{array}{l}\mathscr{2} \\
\infty \\
\infty\end{array}$ & $\underset{\substack{R \\
\infty}}{\substack{\infty \\
\infty}}$ & $\begin{array}{l}\stackrel{8}{\infty} \\
\infty \\
\infty\end{array}$ & ஜू̆ & ?̊̊ & $\frac{n}{a}$ \\
\hline 品 & $\begin{array}{l}0 \\
n \\
\infty \\
1 \\
1\end{array}$ & \begin{tabular}{l}
8 \\
\multirow{2}{0}{} \\
o \\
1 \\
1
\end{tabular} & $\begin{array}{l}8 \\
6 \\
\infty \\
1 \\
1\end{array}$ & 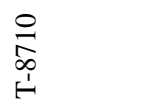 & 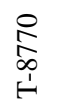 & $\begin{array}{l}8 \\
\infty \\
\infty \\
\infty \\
i\end{array}$ & $\begin{array}{l}\stackrel{R}{2} \\
\infty \\
\infty \\
i \\
1\end{array}$ & $\begin{array}{l}\stackrel{8}{\infty} \\
\infty \\
\infty \\
1 \\
1\end{array}$ & 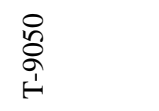 & 尽 & $\frac{i n}{a}$ \\
\hline
\end{tabular}




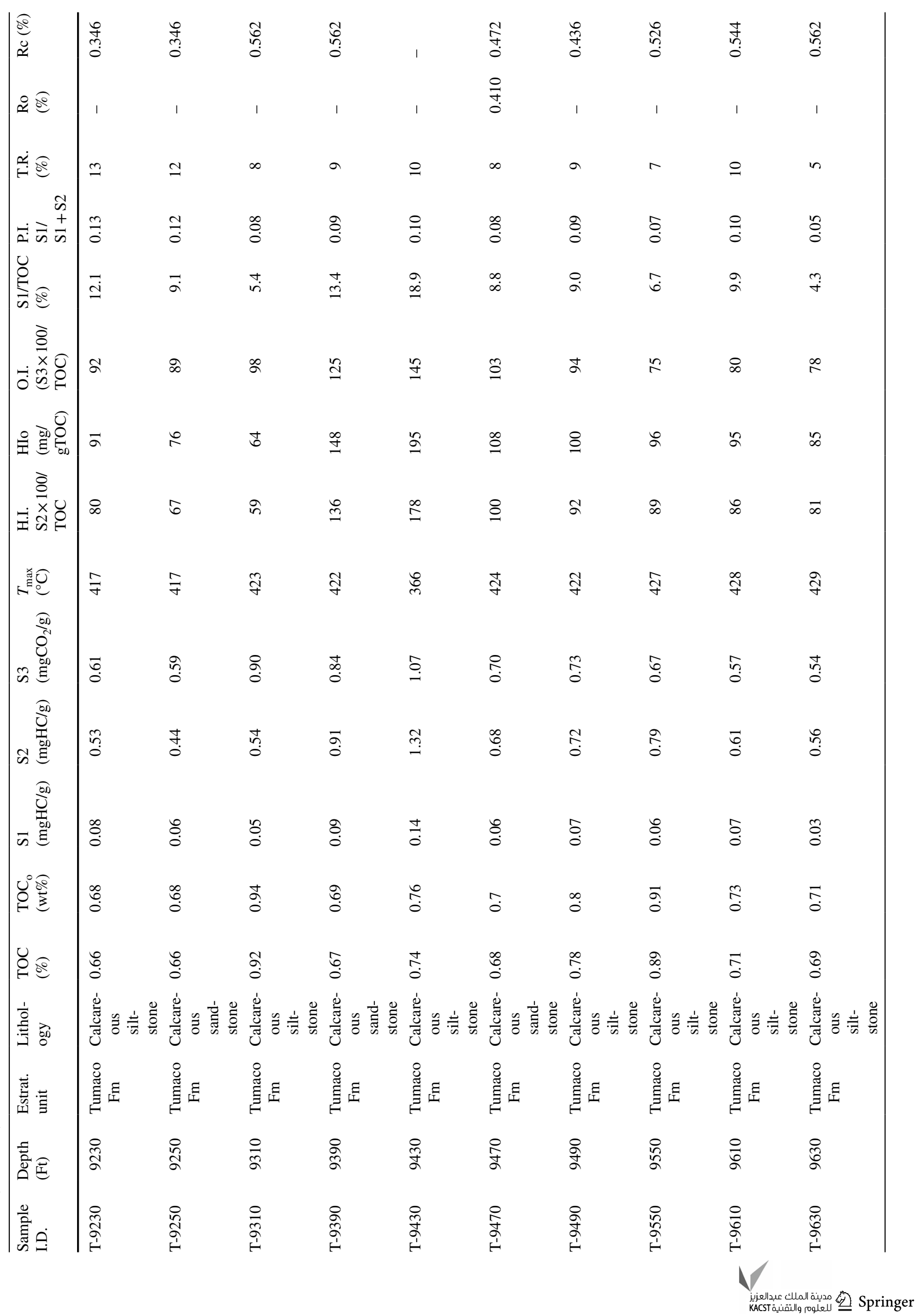




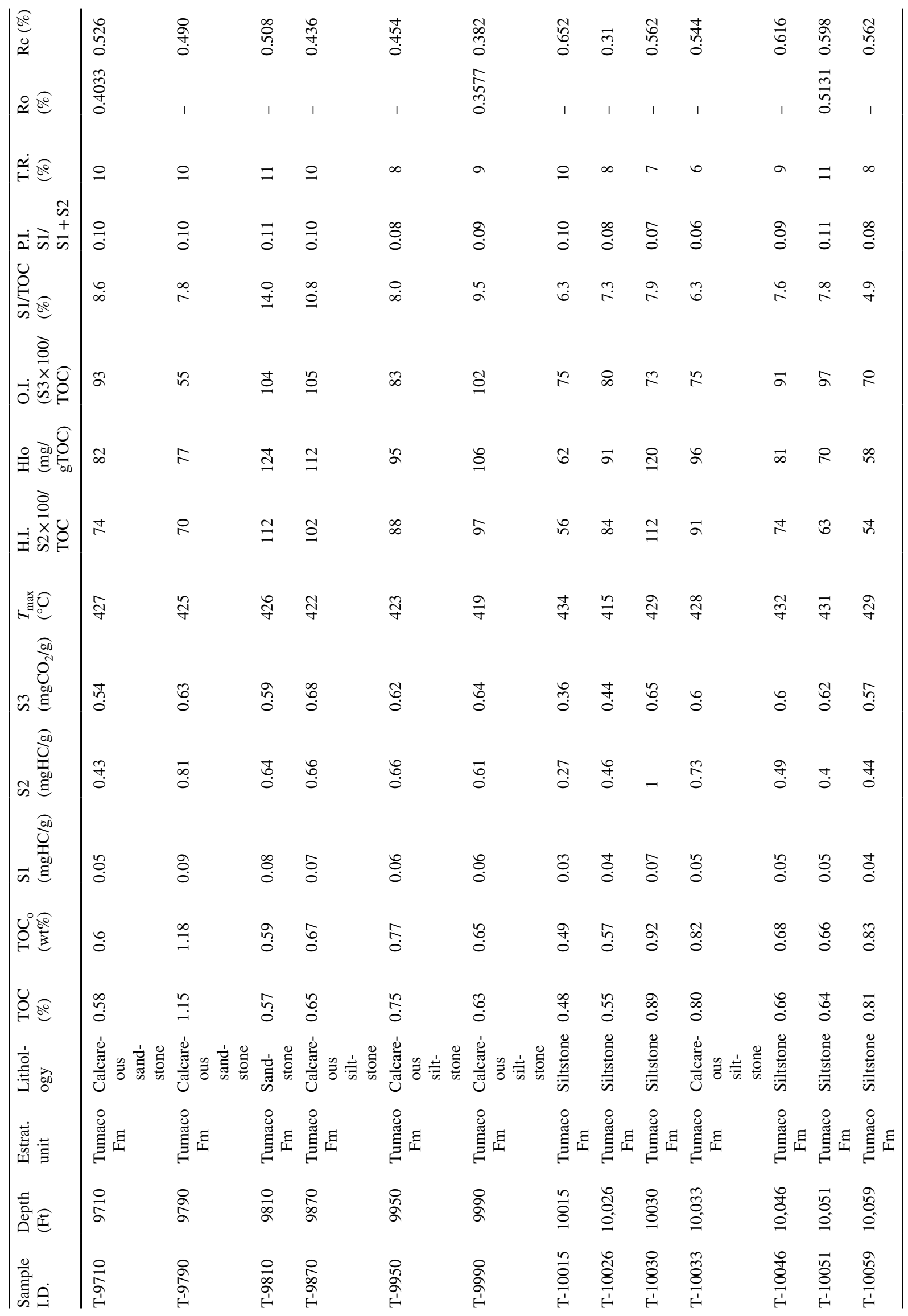




\begin{tabular}{|c|c|c|c|c|c|c|c|c|c|c|c|c|c|c|c|c|c|c|}
\hline $\begin{array}{l}0 \\
\tilde{a}\end{array}$ & $\begin{array}{l}\infty \\
n \\
0 \\
0\end{array}$ & $\begin{array}{l} \pm \\
0 \\
0 \\
0\end{array}$ & $\begin{array}{l}\text { ర్ర } \\
\stackrel{2}{0}\end{array}$ & $\stackrel{0}{0}$ & $\stackrel{0}{0}$ & $\stackrel{0}{0}$ & 点 & \begin{tabular}{l}
\multirow{1}{0}{} \\
ర0. \\
0
\end{tabular} & $\stackrel{\infty}{n}$ & $\begin{array}{l}0 \\
\stackrel{0}{0} \\
0\end{array}$ & 节 & \begin{tabular}{l}
$\infty$ \\
\multirow{2}{0}{} \\
0
\end{tabular} & ڤ్ & $\stackrel{\infty}{n}$ & \begin{tabular}{l}
\multirow{+}{0}{} \\
: \\
0
\end{tabular} & $\begin{array}{l}0 \\
0 \\
0 \\
0\end{array}$ & ڤ్ & ڤ̊ \\
\hline$\approx \precsim$ & 1 & 1 & 1 & $\begin{array}{l}\overline{\hat{o}} \\
\dddot{0}\end{array}$ & 1 & 1 & 1 & 1 & $\begin{array}{l}+ \\
\infty \\
\infty \\
0\end{array}$ & 1 & 1 & 1 & 1 & $\begin{array}{l}0 \\
0 \\
0 \\
0\end{array}$ & 1 & 1 & 1 & 1 \\
\hline so & $n$ & in & $r$ & 0 & $\infty$ & $a$ & $r$ & $r$ & $\infty$ & $\infty$ & $n$ & $r$ & $r$ & $r$ & $r$ & $r$ & $\infty$ & $\infty$ \\
\hline 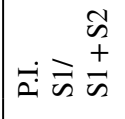 & $\stackrel{n}{0}$ & $\stackrel{n}{0}$ & $\stackrel{5}{0}$ & $\stackrel{\circ}{\circ}$ & $\stackrel{\infty}{0}$ & $\stackrel{\circ}{0}$ & $\stackrel{5}{0}$ & $\stackrel{5}{0}$ & $\stackrel{\infty}{0}$ & $\stackrel{\infty}{0}$ & $\stackrel{2}{0}$ & $\stackrel{5}{0}$ & $\stackrel{0}{0}$ & $\stackrel{5}{0}$ & $\stackrel{5}{0}$ & $\stackrel{5}{0}$ & $\stackrel{\infty}{0}$ & $\stackrel{\infty}{\circ}$ \\
\hline$\underset{5}{\infty}$ & $\stackrel{\infty}{+}$ & $\stackrel{\ddot{n}}{ }$ & $\ddot{n}$ & $\stackrel{\infty}{m}$ & ? & $\stackrel{?}{\sim}$ & $\stackrel{\leftrightarrow}{\dot{f}}$ & $\stackrel{r}{\dot{f}}$ & $\stackrel{0}{i}$ & $\stackrel{\infty}{i}$ & $\stackrel{\sim}{+}$ & $\stackrel{\leftrightarrow}{\dot{f}}$ & $\stackrel{\check{f}}{\text { r }}$ & in & $\ddot{n}$ & $\stackrel{\infty}{n}$ & $\stackrel{n}{r}$ & $\stackrel{b}{i}$ \\
\hline 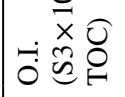 & $\stackrel{\infty}{\curvearrowleft}$ & 6 & $\infty$ & $\bar{\infty}$ & $\underset{O}{\stackrel{d}{0}}$ & さे & 8 & $\stackrel{\infty}{\sim}$ & $\kappa$ & $\infty$ & $\approx$ & $\hat{6}$ & $\stackrel{\varrho}{\varrho}$ & $\Re$ & $\stackrel{2}{\sim}$ & $\widehat{6}$ & a & $\stackrel{\infty}{\circ}$ \\
\hline 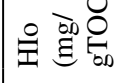 & ฉ & \& & f & 8 & $\underset{\infty}{+}$ & $\stackrel{\infty}{\sim}$ & 8 & $F$ & $\widehat{ర}$ & $\cong$ & 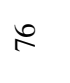 & 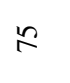 & 8 & $\cong$ & న & $\triangleright$ & $\infty$ & $\stackrel{2}{2}$ \\
\hline 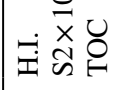 & a & $\bar{\sigma}$ & 列 & in & $\stackrel{\infty}{\sim}$ & $\approx$ & $\widehat{\sigma}$ & 8 & in & 8 & $\approx$ & $R$ & $\vec{\sigma}$ & $\infty$ & $\therefore$ & $\approx$ & $\bar{\infty}$ & g \\
\hline N & $\vec{F}$ & $\ddot{q}$ & ఫे & $\widetilde{F}$ & $\widetilde{ซ}$ & $\underset{ซ ్}{ซ}$ & $\ddot{\vartheta}$ & 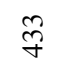 & $\stackrel{\oplus}{+}$ & $\underset{ซ}{ซ}$ & $\ddot{\vartheta}$ & $\overrightarrow{\tilde{r}}$ & $\vec{q}$ & $\stackrel{\overbrace{}}{\mathscr{f}}$ & $\stackrel{m}{q}$ & $\stackrel{\widetilde{F}}{\mathscr{\gamma}}$ & $\vec{\xi}$ & $\vec{F}$ \\
\hline$\tilde{n}$ & gे. & $\stackrel{8}{0}$ & $\because$ & $\stackrel{n}{0}$ & $\stackrel{\infty}{+}$ & $\tilde{n}$ & $\stackrel{t}{0}$ & $\stackrel{8}{\circ}$ & $\begin{array}{l}0 \\
? \\
0\end{array}$ & $\stackrel{n}{\text { fo }}$ & f̊. & กี & $\stackrel{0}{0}$ & $\stackrel{\infty}{n}$ & $\sqrt[n]{0}$ & $\stackrel{0}{0}$ & $\stackrel{8}{\circ}$ & $\tilde{n}$ \\
\hline$\tilde{\sigma}$ & $\stackrel{\hat{\imath}}{0}$ & $\stackrel{\vec{\sigma}}{-}$ & ๙ุ & f & ़ֻ & స్ & $\stackrel{\infty}{\infty}$ & $\stackrel{?}{0}$ & ले & ֻొ & กิ & $\stackrel{n}{n}$ & ఠે & กี & $\stackrel{+}{n}$ & กี่ & th & $\stackrel{0}{0}$ \\
\hline $\bar{n}$ 兑 & $\stackrel{t}{0}$ & $\stackrel{0}{0}$ & 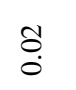 & $\stackrel{8}{0}$ & $\stackrel{8}{0}$ & $\stackrel{8}{0}$ & 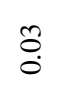 & $\stackrel{t}{0}$ & $\stackrel{0}{0}$ & $\stackrel{0}{0}$ & $\stackrel{0}{0}$ & $\stackrel{t}{0}$ & $\stackrel{m}{0}$ & $\stackrel{t}{0}$ & $\stackrel{+}{0}$ & $\stackrel{t}{0}$ & $\stackrel{n}{0}$ & $\stackrel{0}{0}$ \\
\hline 员 & $\begin{array}{l}\infty \\
\stackrel{0}{0}\end{array}$ & $\stackrel{\vartheta}{\sigma}$ & n̊? & $\stackrel{\substack{0 \\
0}}{0}$ & f̊. & $\stackrel{\text { f }}{0}$ & $\stackrel{0}{0}$ & $\stackrel{\infty}{\infty}$ & $\stackrel{0}{0}$ & $\tilde{n}$ & $\stackrel{t}{\stackrel{0}{0}}$ & $\stackrel{+}{+}$ & $\stackrel{\bullet}{\circ}$ & $\stackrel{2}{0}$ & $\stackrel{\mathbb{2}}{\stackrel{0}{0}}$ & $\stackrel{i}{0}$ & ô & $n$ \\
\hline 0 & 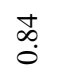 & $\underset{乛}{\stackrel{8}{-}}$ & $n$ & $\stackrel{\infty}{\infty}$ & I্ & F & $\stackrel{\bullet}{0}$ & $\stackrel{n}{\infty}$ & $\stackrel{8}{0}$ & กี่ & $\stackrel{0}{0}$ & $\stackrel{\substack{0 \\
0}}{0}$ & ț & $\stackrel{\hat{a}}{0}$ & $\stackrel{?}{:}$ & $\stackrel{0}{0}$ & ڤ్ర & tr \\
\hline 总 & $\sum_{\Sigma}^{+}$ & 害总 & 弄 竞 & $\sum_{\Sigma}^{\frac{1}{z}}$ & $\sum_{\Sigma}^{\frac{1}{z}} \frac{0}{0}$ & $\sum_{\Sigma}^{\frac{1}{\Xi}}$ & $\sum_{\Sigma}^{\dot{g}}$ & 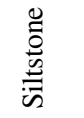 & 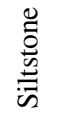 & 总 & 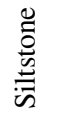 & 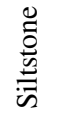 & 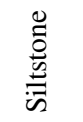 & $\frac{\mathscr{0}}{\stackrel{0}{\underline{m}}}$ & 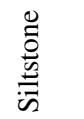 & 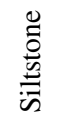 & 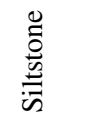 & 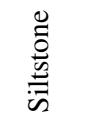 \\
\hline 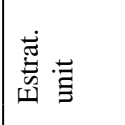 & $\stackrel{\mathscr{\Xi}}{\dddot{g}}$ & 兽 & $\stackrel{8}{\stackrel{8}{\leftrightarrows}}$ & 兽 & 兽国 & 兽 & 兽匡 & 兽 & 兽 & 总 & 兽 & 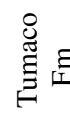 & 惫焉 & 兽国 & 总国 & 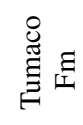 & 总 & 兽医 \\
\hline 言 & $\begin{array}{l}\hat{8} \\
\stackrel{0}{0}\end{array}$ & $\begin{array}{l}\hat{\hat{O}} \\
\hat{0}\end{array}$ & $\begin{array}{l}\hat{\infty} \\
\stackrel{0}{0} \\
\stackrel{0}{0}\end{array}$ & $\begin{array}{l}\infty \\
\stackrel{0}{0} \\
\stackrel{0}{9}\end{array}$ & $\stackrel{2}{\varrho}$ & $\stackrel{2}{\dddot{g}}$ & $\begin{array}{l}\stackrel{\leftrightarrow}{0} \\
\stackrel{0}{0}\end{array}$ & $\frac{\mathfrak{g}}{\mathrm{g}}$ & $\begin{array}{l}\text { రె } \\
0 \\
0\end{array}$ & $\stackrel{n}{=}$ & $\stackrel{+}{\infty}$ & $\frac{0}{2}$ & $\begin{array}{l}\text { ᄋे } \\
\text { ○े }\end{array}$ & $\begin{array}{l}\text { तె } \\
\stackrel{9}{\varrho}\end{array}$ & 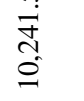 & $\begin{array}{l}\sqrt{3} \\
\stackrel{0}{=}\end{array}$ & $\begin{array}{l}\text { ते } \\
\text { @े }\end{array}$ & $\begin{array}{l}\text { oे } \\
\text { ․ } \\
0\end{array}$ \\
\hline$\stackrel{0}{z}$ & $\frac{\hat{b}}{0}$ & $\frac{\hat{\sigma}}{8}$ & $\begin{array}{l}\infty \\
8 \\
0 \\
1 \\
1\end{array}$ & 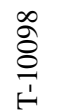 & $\frac{2}{0}$ & $\frac{a}{a}$ & $\frac{a}{a}$ & $\frac{\stackrel{N}{\sigma}}{\frac{\mathfrak{I}}{1}}$ & 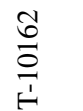 & $\frac{n}{a}$ & $\frac{+}{\infty}$ & $\frac{\varrho}{\circ}$ & 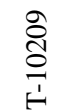 & 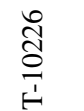 & 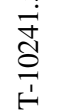 & 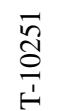 & 旤 & 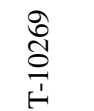 \\
\hline
\end{tabular}




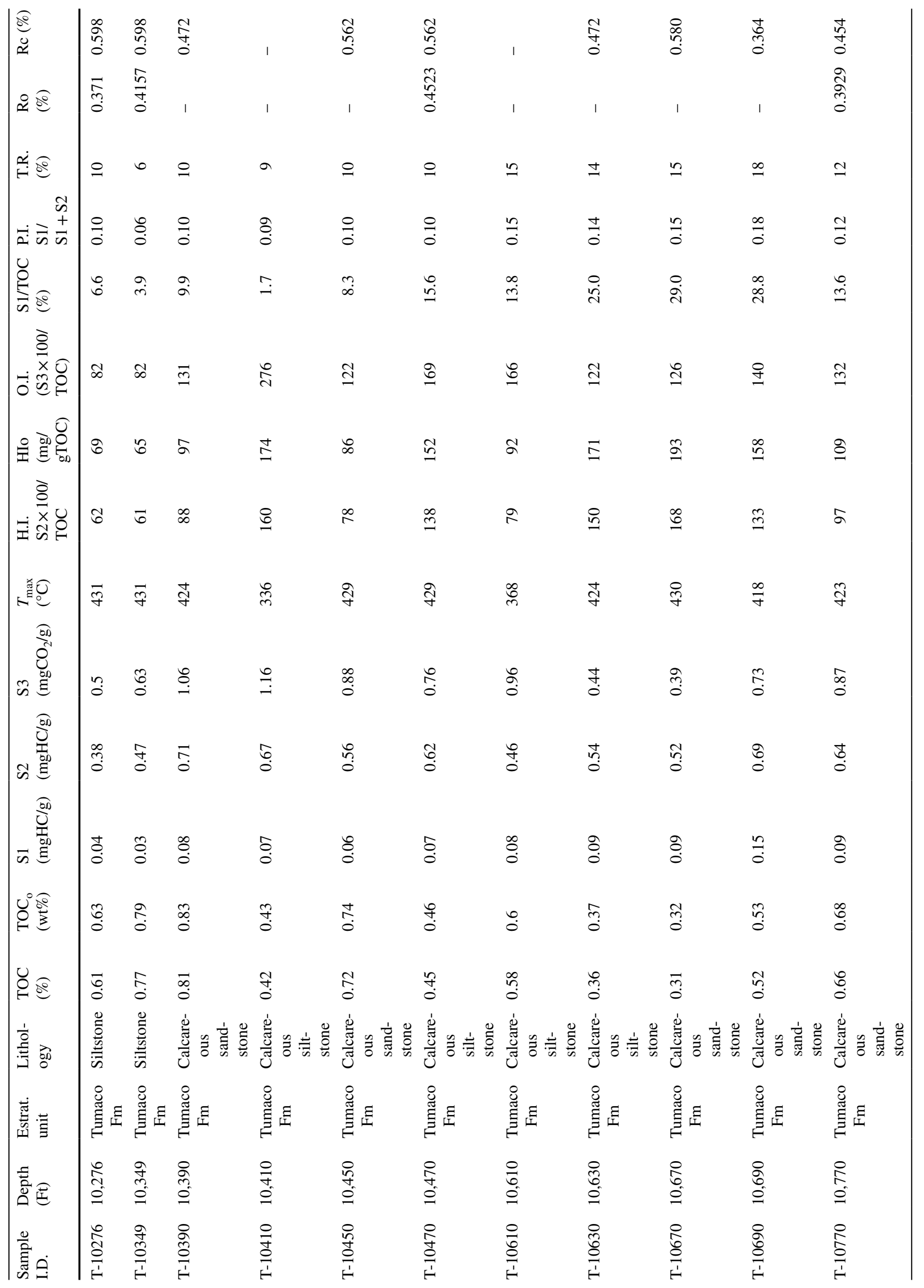




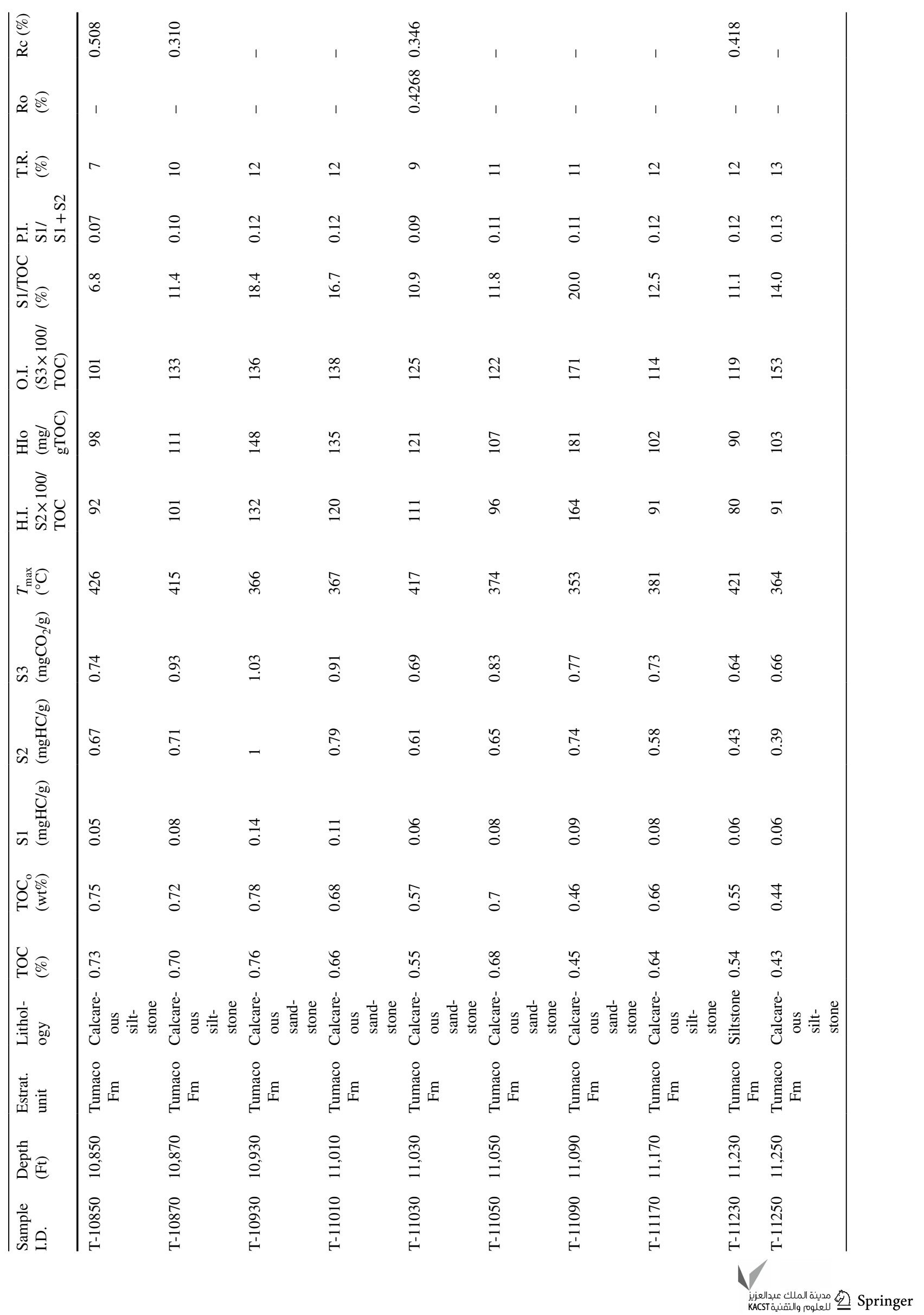




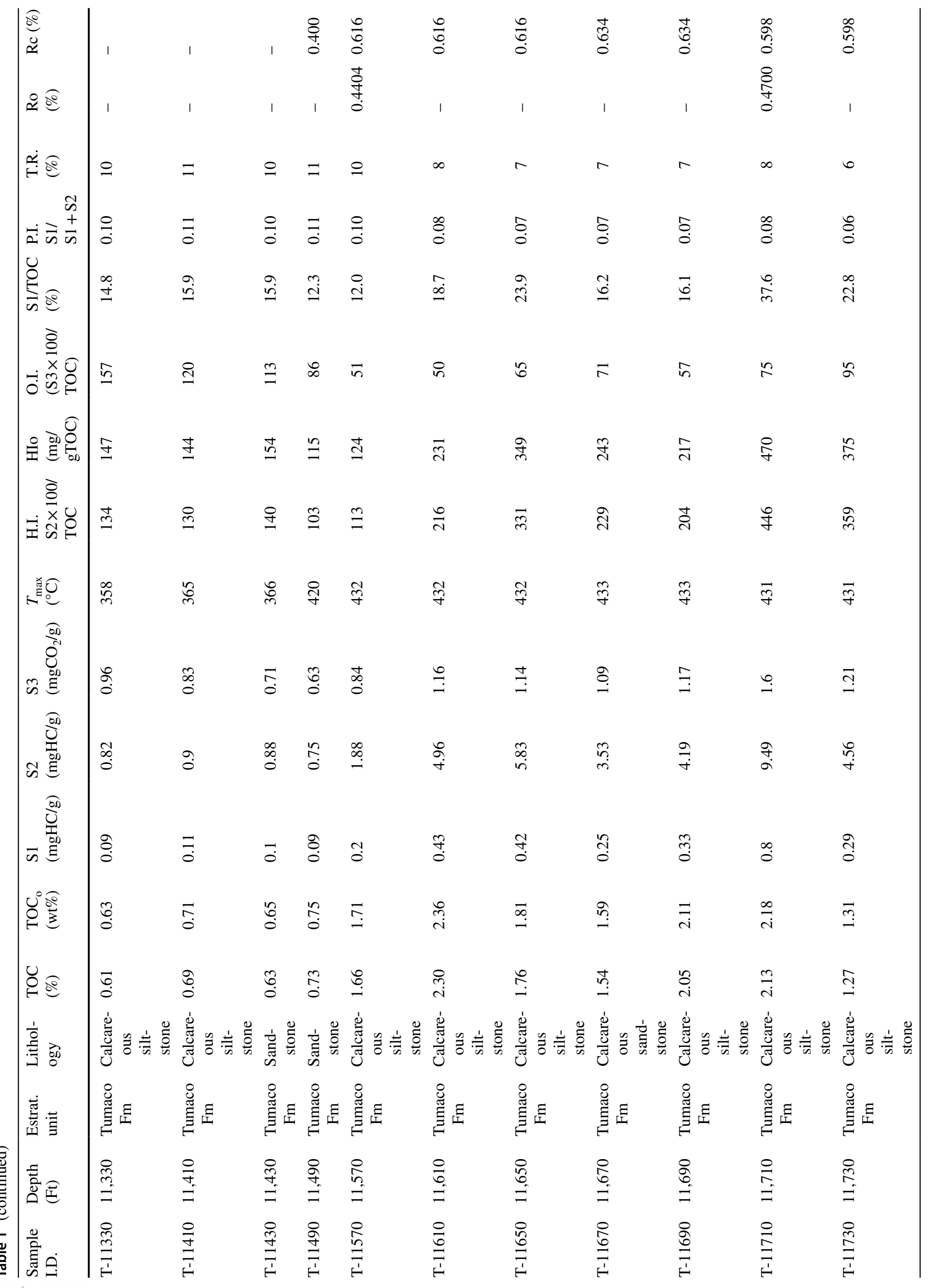


Journal of Petroleum Exploration and Production Technology (2019) 9:1747-1800

1773

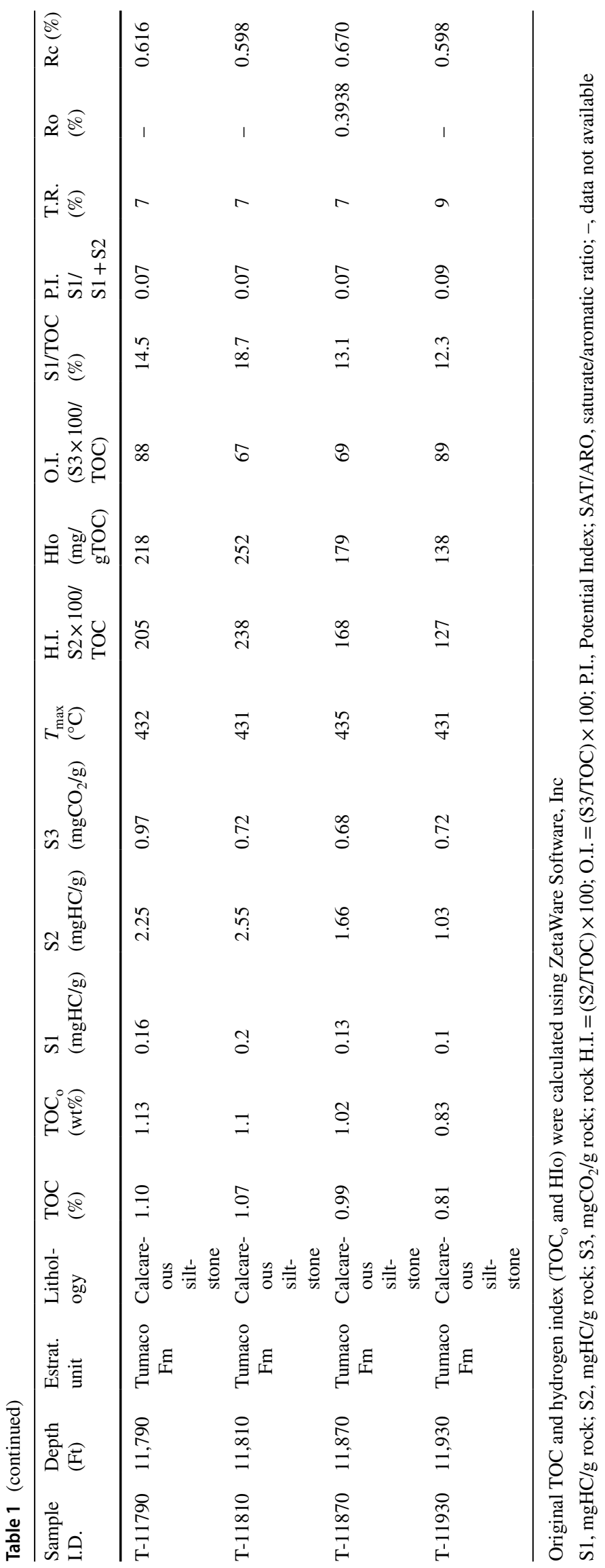


Fig. 3 Modified van Krevelen diagram based on IH versus IO for Tumaco-ST-P well, Tumaco basin, Colombia. $\mathrm{HI}=(\mathrm{S} 2 / \mathrm{TOC}) \times 100, \mathrm{OI}=(\mathrm{S} 3 /$ TOC) $\times 100$

Fig. 4 Kerogen type and thermal maturity using $\mathrm{HI}(\mathrm{mg}$ $\mathrm{HC} / \mathrm{g}$ TOC) versus $T_{\max }\left({ }^{\circ} \mathrm{C}\right)$
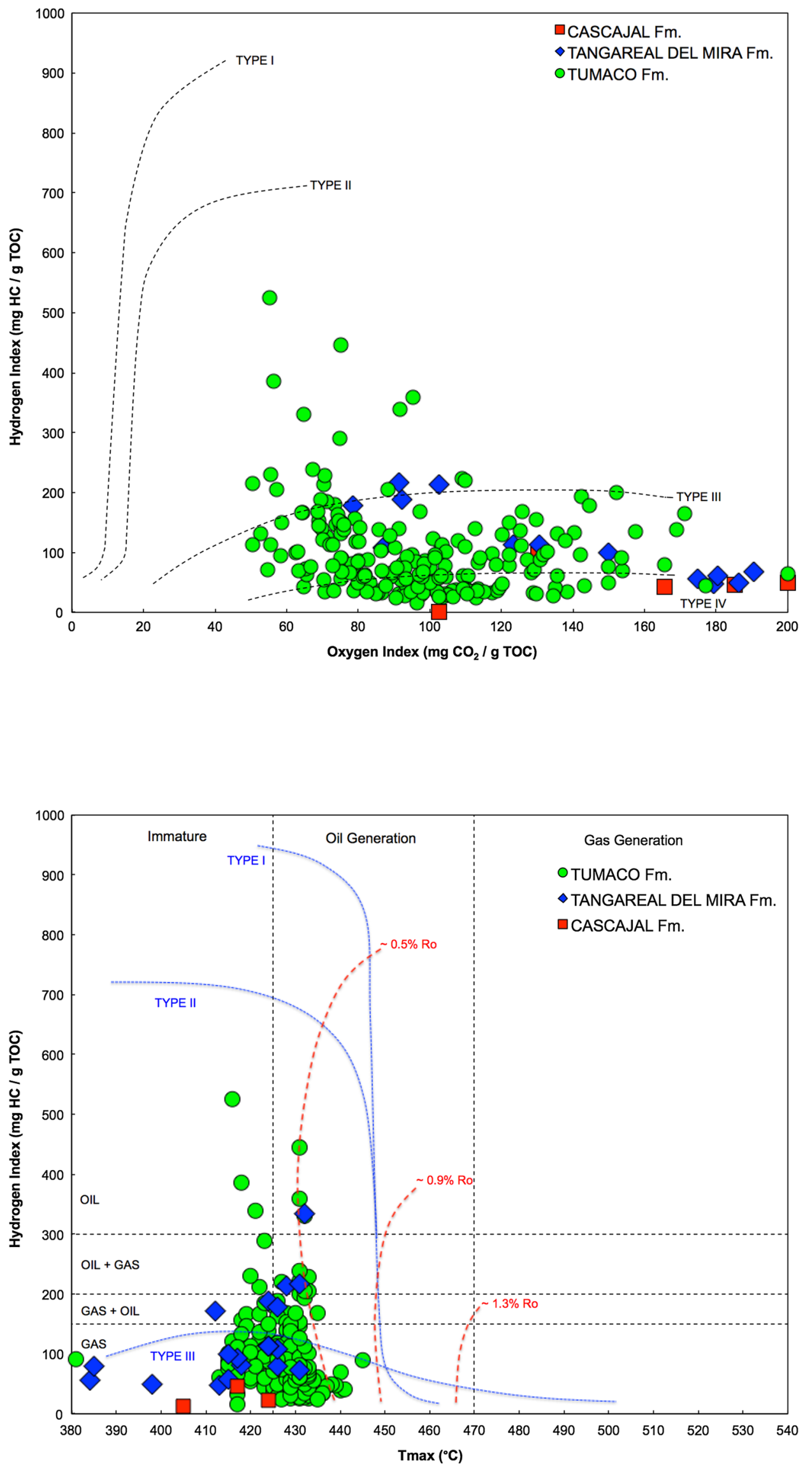
Fig. 5 Kerogen type (pseudo van Krevelen diagram) using $\mathrm{S} 2$ peak (mg HC/g rock) versus present-day TOC, $\%$

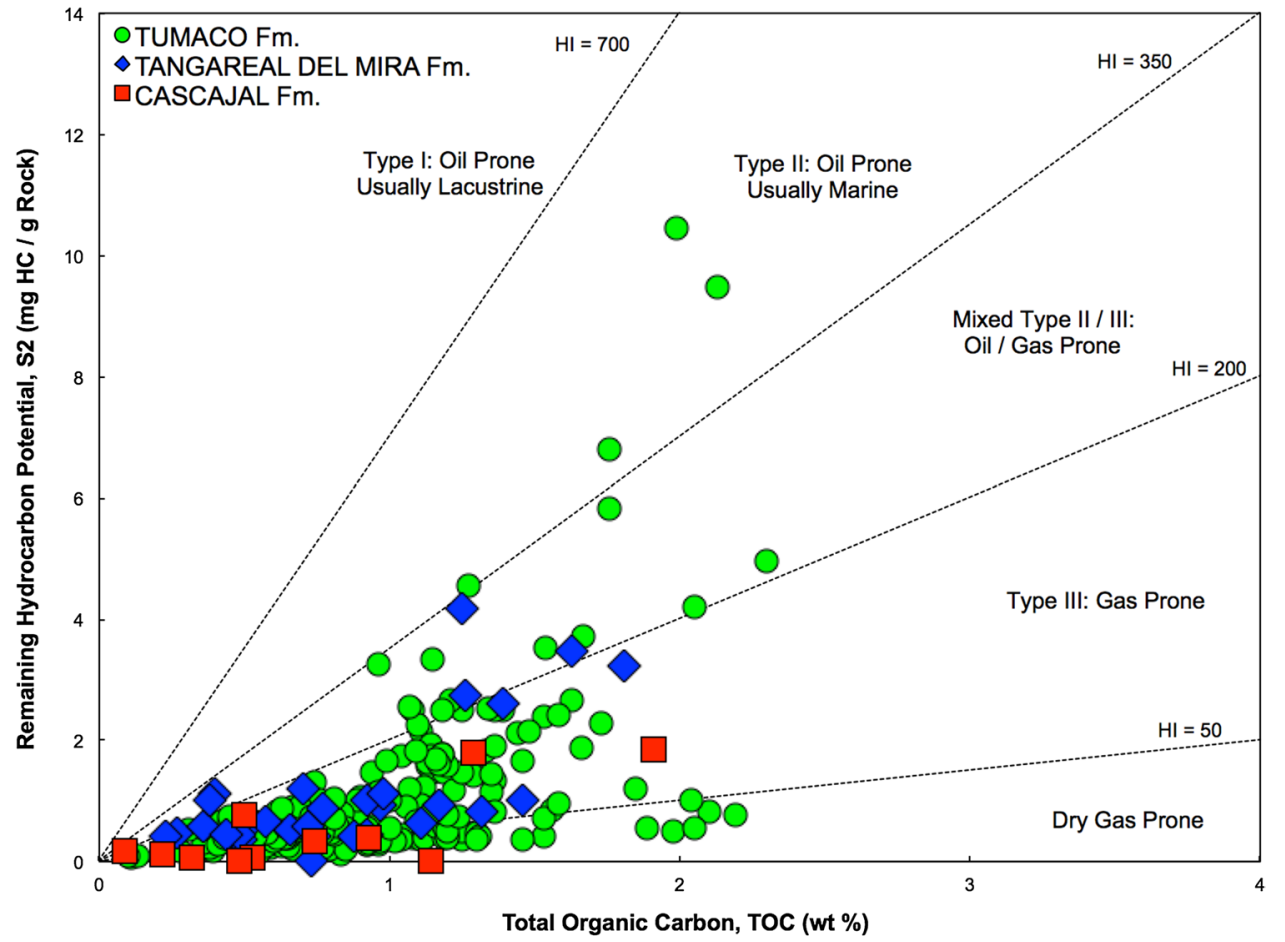

oil and reported in this paper as Ro (Pawlewicz and Finn 2013). Additionally, the Rc was calculated from $T_{\max }$ (Jarvie et al. 2001). Although this approach works better when $T_{\max }$ is between 420 and $500{ }^{\circ} \mathrm{C}$, it has been used in this study due to this conversion works marginally well for type III kerogen which includes the most of the analyzed samples. Fifty samples were selected through the stratigraphic column for Ro analysis in order to better understand its thermal maturity and hydrocarbons potential. The average Ro value was $0.4046 \pm 0.065 \%$ indicating an immature O.M.

Macerals are the individual components of the kerogen that have petrographically and geochemically distinct properties and an independent assessment of the quality, quantity, and thermal maturity of organic matter (Hall et al. 2016). Vitrinite, liptinites, and inertinites were the most common maceral groups found in the dispersed organic matter in Tumaco-1-ST-P samples, which in turn allow to identify the following maceral types: esporinite, cutinite, resinite, alginite, colotelinite, colodetrinite, funginite, exsudinite, exsudatinite, inertodetrinite, among others as is shown in the photomicrographs of Fig. 7. The origin of macerals identified has a resin of higher plants, spores/pollen, cuticular plants, algae, and mixtures of the above. These macerals come from type II kerogen, while the vitrinite identified in relatively high concentration comes from type III kerogen and probably originated from tissues of wood and other vegetables. The inertinite that groups types of macerals as nertodecrinite, fusinite, etc., could originate from wood carbonized fragments. The petrographic analysis indicates that the rocks studied are in immature state. Figure 8 shows the plot of depth (ft) versus Ro $(\%)$ for modeling of the evolution of the thermal maturity. However, rocks with O.M. in the hydrocarbon generation window can exist at $1700 \mathrm{ft}$ in stratigraphic column between 950 and 11,900 ft

The variation of Ro with depth and the petrographic analysis indicates that almost all samples in the Tumaco-1-ST-P well are in the immature state where samples from Cascajal and Tangareal del Mira Fms show the lowest maturity, while the highest maturities were found in the Tumaco Fm. suggesting a consistent light increment of maturity with depth. This linear regression with confidence bounds of $95 \%$, suggests a depth range up to approximately 17,000 feet (see ellipse) where it is expecting that the potential source rock will enter into the generation window for Tumaco Basin, Colombia.

To estimate the extension of the petroleum-generation process and the total generative capacity in an already mature source rock, information on the TOC and HI of organic matter prior to the onset of hydrocarbon generation is required (Jarvie 2012, Hall et al. 2016). Although TOCpd and $\mathrm{HI}_{\mathrm{pd}}$ classified the organic matter deposited in Tumaco 1-ST-P as immature for what one could assume that $\mathrm{HI}_{\mathrm{pd}}$ should be equal HIo (Jarvie 2012), we have used KinEx source rock modeling software (www.zetaware.com) to calculate the original TOC and HI based on present-day analyses (Table 1). The minimum differences in the original and present-day TOC and HI confirm that the O.M. in Tumaco-1-ST-P is very immature and that the data could be

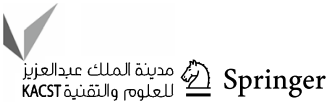


Fig. 6 Lithology, paleoenvironment and stratigraphic correlation for Tumaco-1-ST-S and

Tumaco-1-ST-P wells (ANHAntek S.A. 2013)

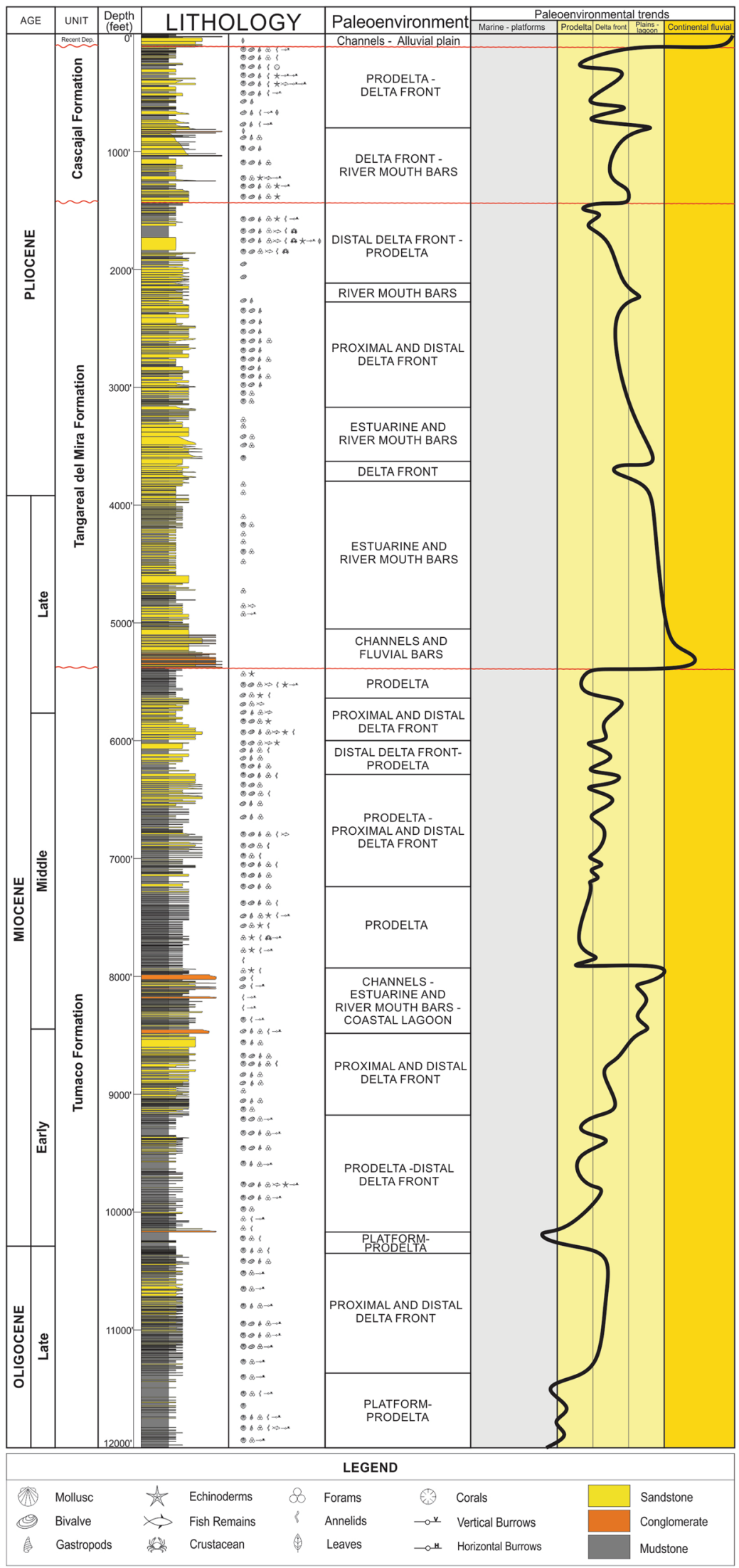


A
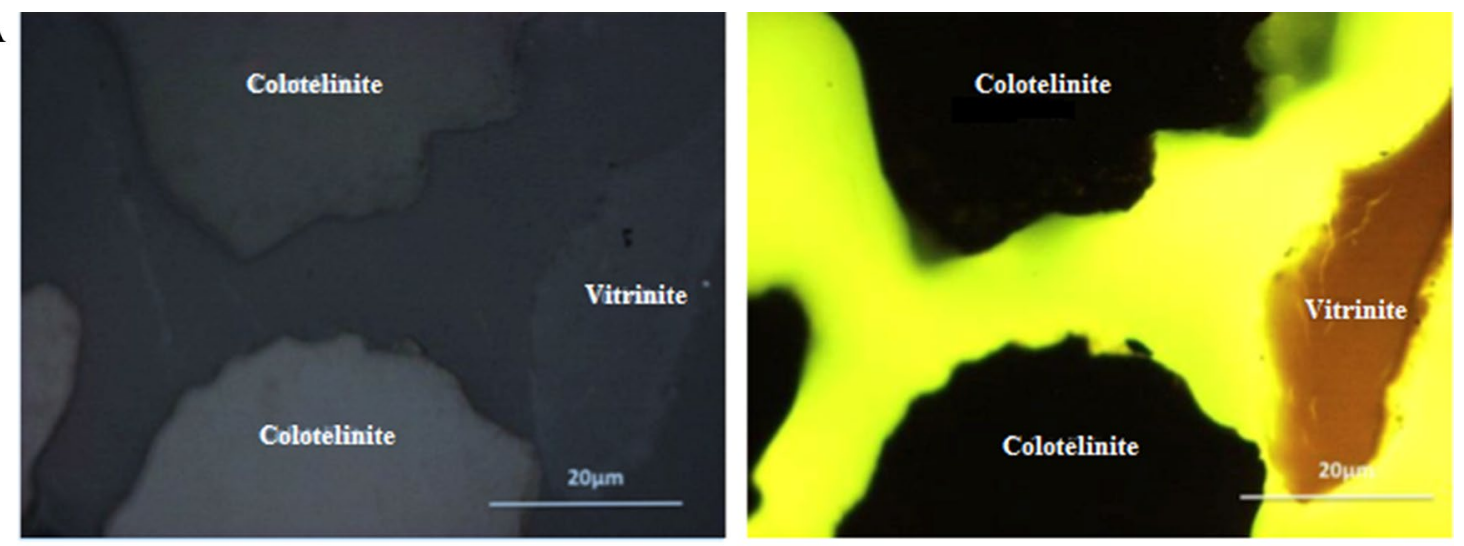

B

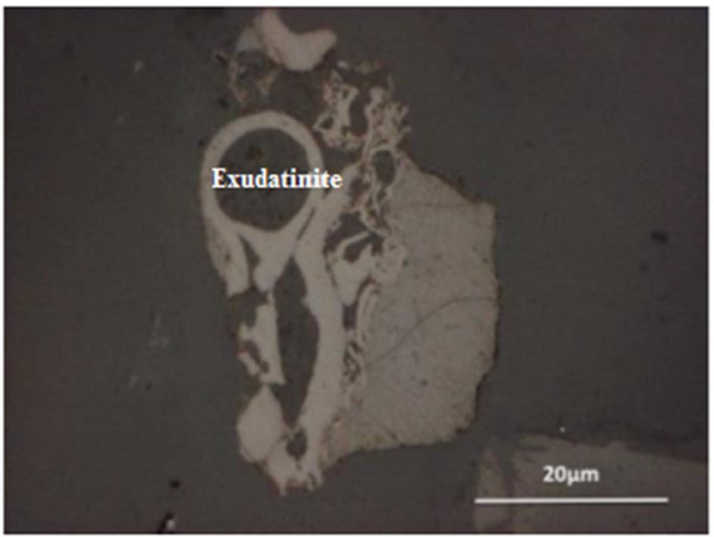

C

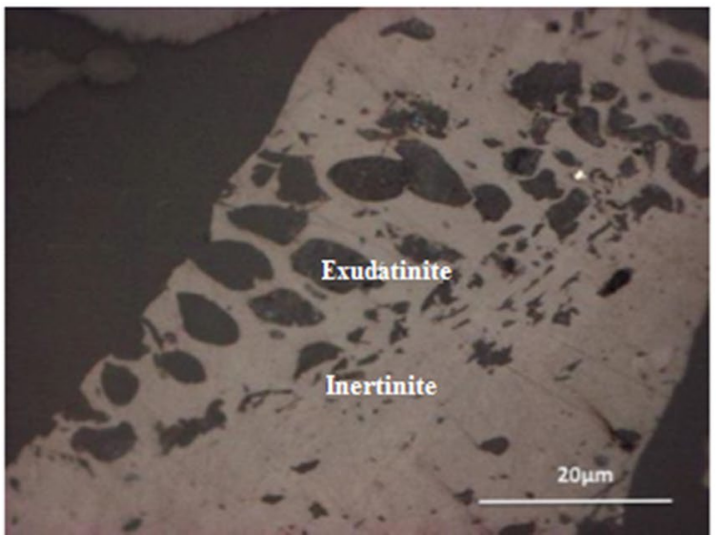

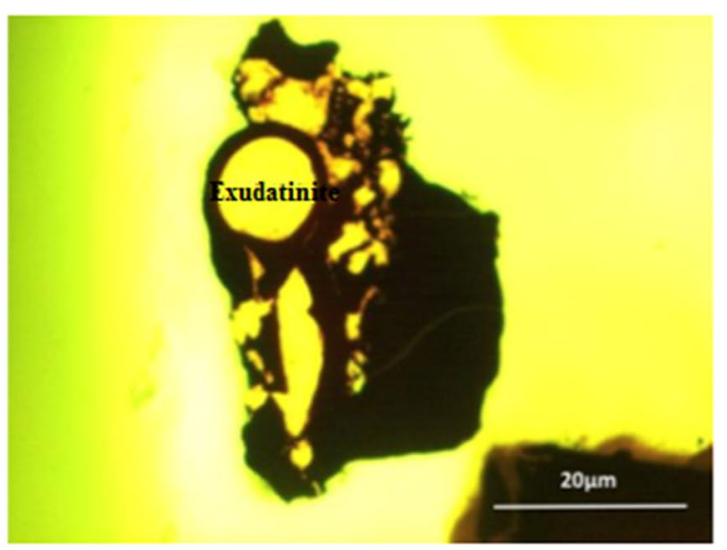

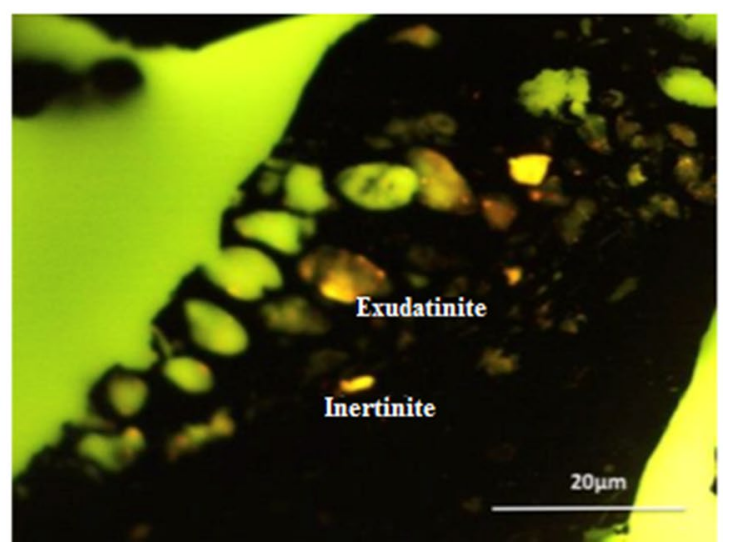

Fig. 7 Photomicrograph in reflected light and fluorescent $(\times 50)$ of sample T-11570. a Grains of vitrinite with different thermal maturities. b Inertinite and exudatinite, and $\mathbf{c}$ inertinite with exudatinite

used to calibrate the maturity in the basin (Jarvie 2012). On average, the HIo (117.57 $\mathrm{mg} \mathrm{HC/g}$ TOC) differs from $\mathrm{HI}_{\mathrm{pd}}$ (109.85 mg HC/g TOC) and is about $7.72 \mathrm{mg} \mathrm{HC/g}$ TOC confirming an immature stage of the O.M. in the sample rocks.

Figure 9 presents an idealized geochemical logging based on screening values (TOC and RE-Py) and the experimental vitrinite reflectance (Ro) associated with lithofacies, stratigraphic units and the age for Tumaco-1-ST-P well applying the molecular stratigraphy concepts, which allows observing the changes of organic facies that occur laterally or vertically into the column (Demaison in Peters 1986; Espitalié et al. 1987; Burwood et al. 1990; Peter et al. 2005). $T_{\max }$ and Ro increase consistently with increasing depth and maturity; $\mathrm{HI}_{\text {pd }}$ shows a few variation with depth in Tumaco Fm, except in the deepest interval where it is consistent with TOCpd contents, indicating a few kerogen conversion to petroleum or gas (low transformation ratios values). 
Fig. 8 Linear regression of depth versus Ro (\%) with confidence bounds of $95 \%$, showing the depth up to approximately 17,000 feet (ellipse) where is expecting that the potential source rock will enter into the generation window for Tumaco Basin, Colombia

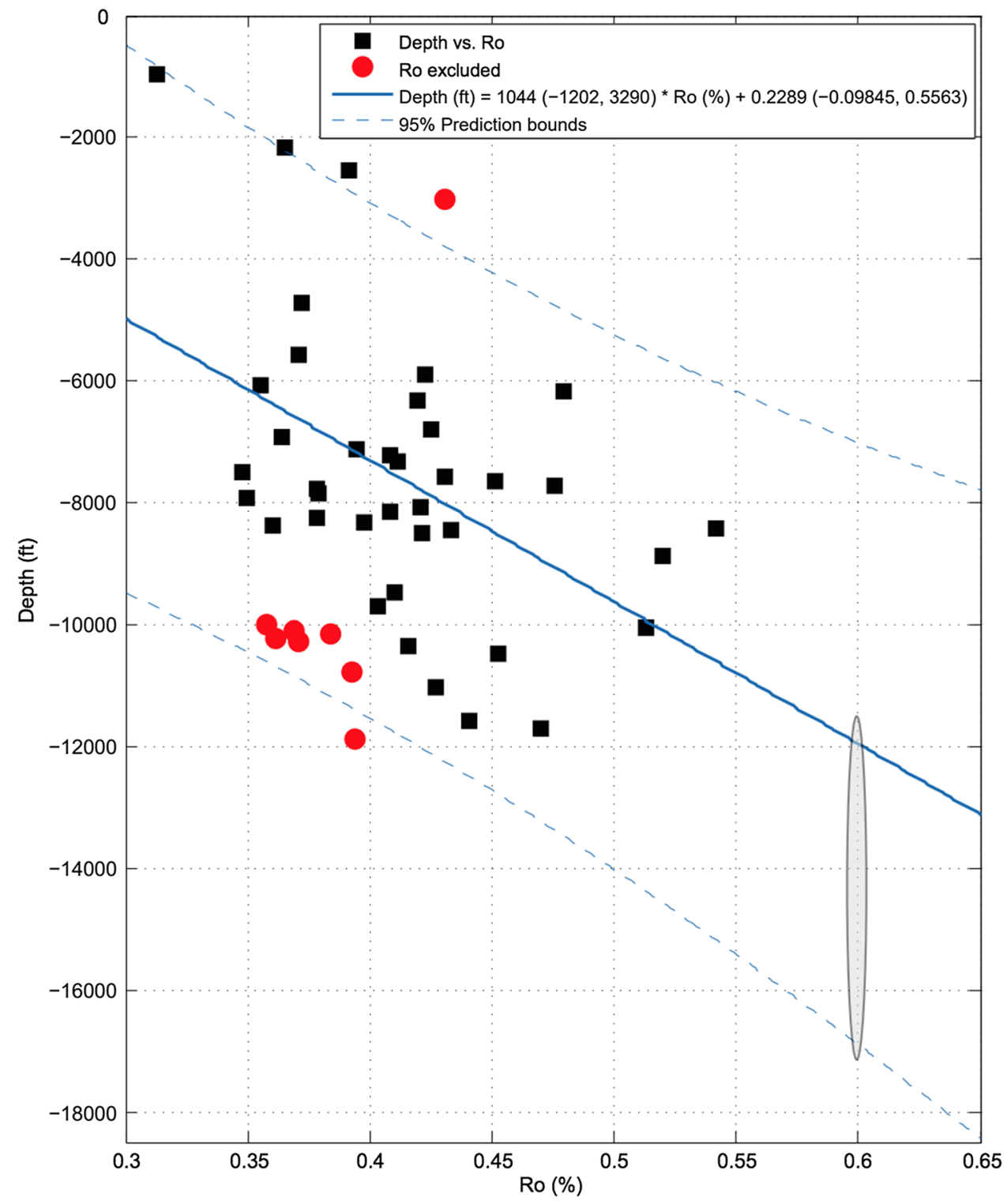

\section{Bitumen extracts and SARA fractionation}

Table 1 also displays organic geochemical characteristics based on EOM \%, SARA, and GC/FID analysis of the bitumen extracted. The EOM is very low, ranging between 0.0018 and $0.75 \%$, being the deepest units of the Tumaco1-ST-P well $(11,650 \mathrm{ft})$ which contains the largest concentrations. Thus, the EOM values characterize the Cascajal and Tangareal del Mira formations as poor, while Tumaco Fm varies between fair and good quality (Bacon et al. 2000). The quantity and composition of the bitumen extracted depend on the original organic matter, organic facies, depositional environment, thermal maturity, and the extension of the expulsion and could also reflect contamination by drilling mud/fluids or migration of hydrocarbons. These last two events are discarded in Tumaco-1-ST-P well due to the low amount of O.M. extracted.

SARA fractionation shows the highest values for the resin fractions (NSO compounds), in most of the organic extracts, varying between 17.47 and $62.33 \%$. Asphaltene fractions vary between 4.65 and $59.02 \%$, and the aromatic fractions range between 0.1 and $21.28 \%$. Finally, the fraction of saturated hydrocarbons varies between 4.99 and $43.81 \%$. Tissot and Welte (1984) proposed a ternary diagram to identify the organic matter type based on the SARA fractionation. Thus, Tumaco-1-ST-P well samples classify as naphthenic bitumen due to its high resin and asphaltene concentrations. The SARA analysis shows a relatively low dispersion on its fraction, indicating that this bitumen has the same source and origin. The saturate/aromatic ratios are in all samples very high, except in the sample T2928 (0.29\%). Thus, the 


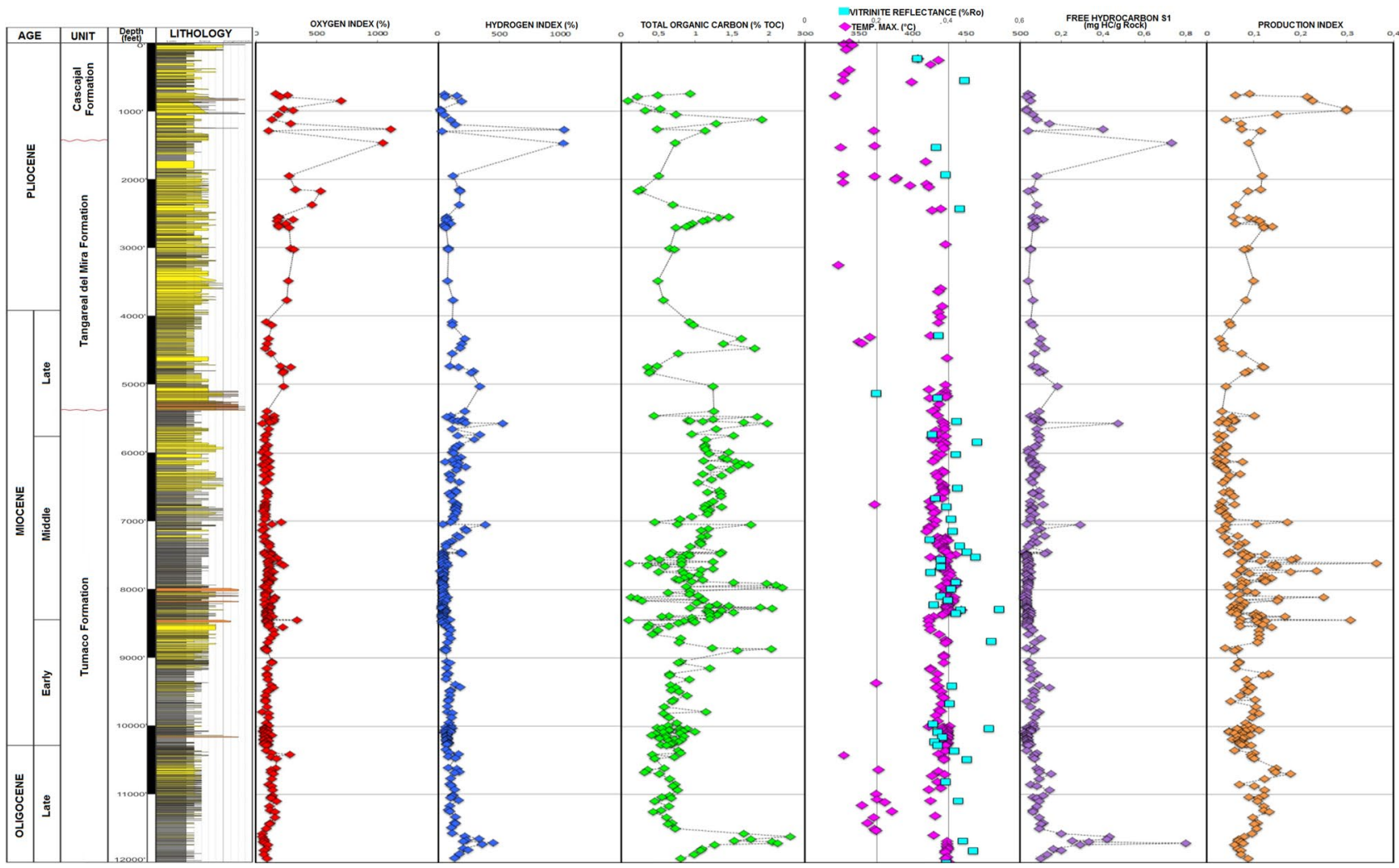

Fig. 9 Idealized geochemical logging based on RE-pyrolysis indexes, TOC and Ro for Tumaco-ST-P, Tumaco basin, Colombia (modified from J.G. Demaison in Peters 1986). Ro is experimental vitrinite reflectance

SAT/ARO ratio indicates a very low content of aromatic hydrocarbons in the entire column and limits the use of the aromatic fraction as maturity indicator as it will be discussed later in Sect. 4.4.2.1.

\section{Source and depositional environment}

\section{$n$-alkanes and isoprenoids}

Gas chromatographic profiles of selected crude oils are shown in Fig. 10. The geochemical ratios based on $n$-alkanes distribution are displayed in Table 2. In general, the $n$-alkanes are the most abundant components in the saturate fraction for all samples (range 4.39-43.81\%) although as stated above saturated hydrocarbons are the major fraction of the bitumen extract. Bitumen extracts have a $n$-alkanes distribution between $n-\mathrm{C}_{10}$ and $n$ - $\mathrm{C}_{33}$, with bimodal distribution and maximum between $n-\mathrm{C}_{15}$ and $n-\mathrm{C}_{17}$, indicating an algal/microbial input and $n-\mathrm{C}_{25}$ to $n$ - $\mathrm{C}_{27}$, showing a significant input of terrigenous organic matter, suggesting that this O.M. was originated from a type II/III or III kerogen. A dominance of $n$-alkanes around of $n$ - $\mathrm{C}_{17}$ is thus interpreted as an indication of a marine source (Jones 1986). The GC profiles for samples T5509 and T-8243 show a special characteristic associated with the presence of high concentration of unknown organic compounds around $n-\mathrm{C}_{29}$ to $n$ $\mathrm{C}_{31}$ and related to recent O.M. (immature) in a process of decomposition and consistent with the concept that many of these samples are in an early diagenetic stage.

A marine-deltaic transitional environment has been strongly supported by the geological model proposed in the original ANH-Antek technical report (ANH-Antek S.A. 2013). The terrestrial origin of a large part of the organic extracts studied comes from the epicuticular wax high plants, which presents a wide distribution of $n$-alkanes with a marked predominance of hydrocarbons with odd number of carbon atoms $\left(n-\mathrm{C}_{25}, n-\mathrm{C}_{27}, n-\mathrm{C}_{29}, n-\mathrm{C}_{31}\right)$; while samples of marine origin located more toward the bottom and in the deepest part of the column have a significant bacterial, algal, and phytoplanktonic contribution, characterized by a predominance of heptadecane $\left(n-\mathrm{C}_{17}\right)$. Some of the chromatographic profiles allow observing a hump or the so-called unresolved complex mixture (UCM), which confirms that different levels of diagenetic biodegradation are in progress in the basin.

The $\mathrm{Pr} / \mathrm{Ph}$ ratio in Tumaco-1-ST-P displays values below 3.0, although most of the samples fall between 1.0 and 1.5, which indicates that these samples were deposited

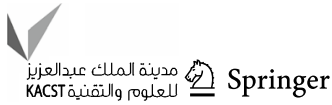



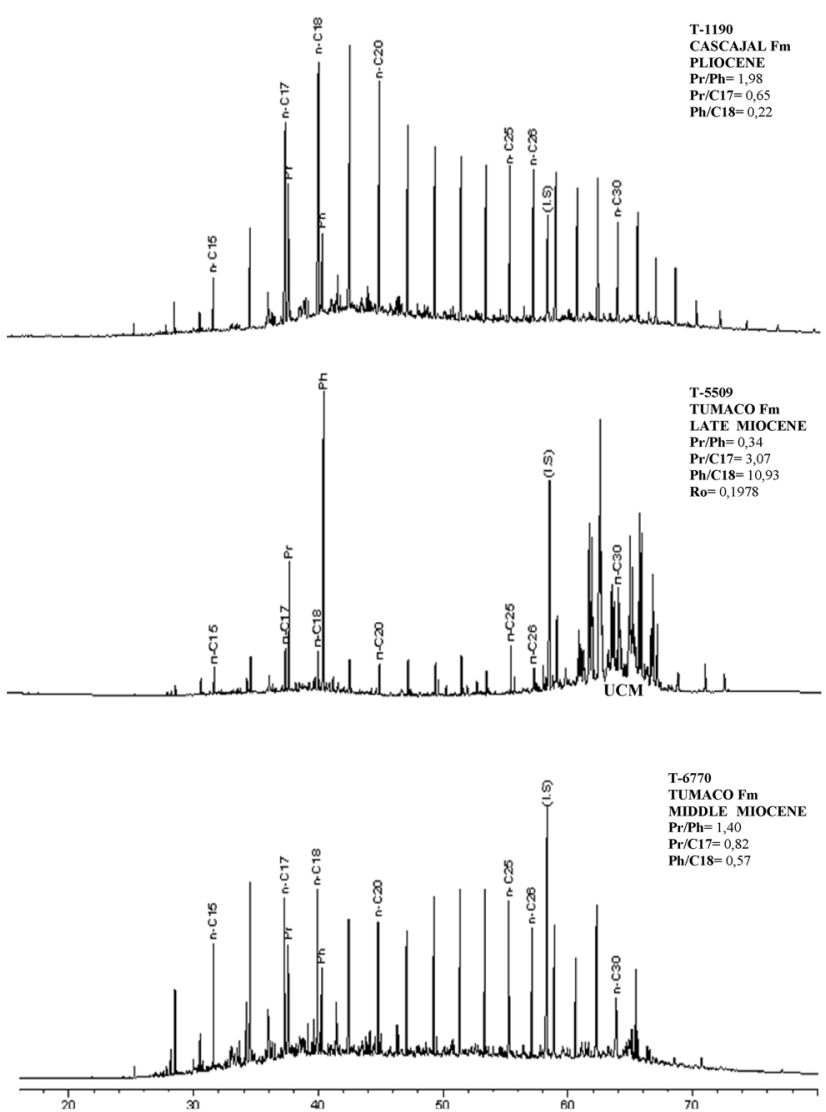
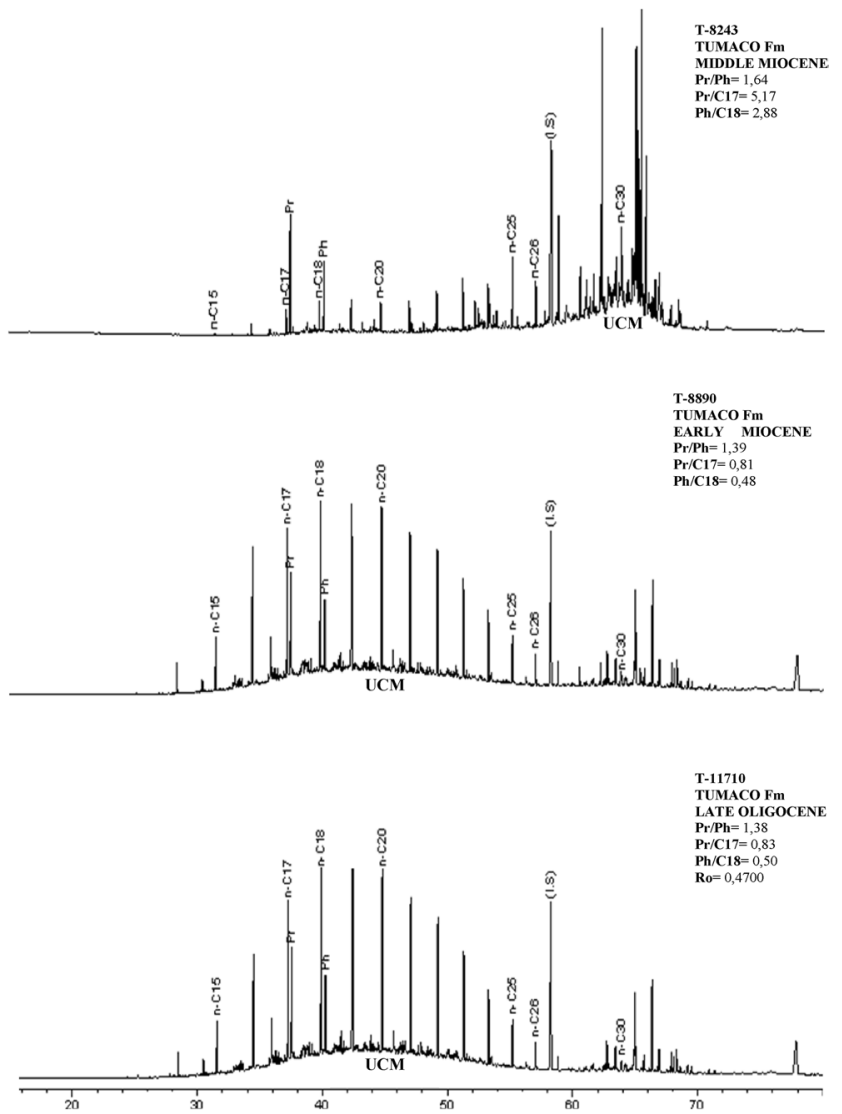

Fig. 10 GC/FID C15 ${ }^{+}$chromatograms of saturate fractions of selected rock extracts in the Tumaco-1-ST-P, Tumaco basin, Colombia

in a suboxic to oxic environment and were derived from marine shales. There are some samples with values of $\mathrm{Pr} / \mathrm{Ph}$ ratio $<1.0$, suggesting a more carbonatic bitumen. Bitumen and crude oils derived from marine shale have a CPI values ranging from low to moderate (1.0-3.0), while those derived from source rocks containing mainly terrestrial O.M. present $\mathrm{Pr} / \mathrm{Ph}$ ratios between 3.0 and 10. Carbonate rocks show $\mathrm{Pr} /$ $\mathrm{Ph}<1.0$ (Didyk et al. 1978). When the $\mathrm{Pr} / \mathrm{Ph}$ is used as an indicator of redox conditions, a value $<3.0$ for the organic extracts of the Tumaco-1-ST-P well, indicates that this O.M. was generated under suboxic-oxic conditions. According to Didyk et al. 1978, $\mathrm{Pr} / \mathrm{Ph}<1$ in bitumen and crude indicates an environment at anoxic depositional environment, while $\mathrm{Pr} / \mathrm{Ph}>1$ indicates an oxic deposition. In Tumaco-1-ST-P rocks, the $\mathrm{Pr} / \mathrm{Ph}$ ratios display values below 3.0, with a high population of data ranging between 1.0 and 1.5 , suggesting that these samples were deposited in a suboxic to an oxic environment.

The $n$-alkanes/isoprenoids ratios $\left(\mathrm{Pr} / n-\mathrm{C}_{17}\right.$ and $\left.\mathrm{Ph} / n-\mathrm{C}_{18}\right)$ decrease with the maturity since more $n$-alkanes are released from kerogen by cracking (Tissot et al. 1971) and are more thermally stable than isoprenoids. Figure 11 shows that most of the samples in Tumaco-1-ST-P well have been originated from type II-III kerogen (transitional marine-terrestrial) and type III kerogen (terrestrial), although a few samples from Tangareal del Mira and Tumaco formations seem to have been originated from a type II kerogen (marine). Thus, $\mathrm{Pr} / n-\mathrm{C}_{17}$ varies between 0.53 and 5.74 and $\mathrm{Ph} / n-\mathrm{C}_{18}$ ranging between 0.22 and 4.88, suggesting a broad range of maturities and biodegradation processes and a depositional environment from suboxic to oxic characteristics.

The carbon preference index (CPI) relates the odd/even hydrocarbon predominance (Bray and Evans 1961). Immature rocks with a significant contribution of superior plants are dominated by odd $n$-alkanes, especially $n-\mathrm{C}_{27}, n-\mathrm{C}_{29}$ and $n-\mathrm{C}_{31}$. These $n$-alkanes are originated from the epicuticular waxy (plant leaves) and are synthesized directly by plants or by defunctionalization from alcohols, acids or esters, in whose case, the CPI values will be $>1.0$. $n$-alkanes derived from marine O.M. tend to have a small or not marked CPI. Regarding to hypersaline carbonates or evaporitic rocks, CPI presents a slight pair preference of numbers of carbon. In all cases, the CPI decreases with the maturity of the O.M. This is due to a geochemical process that combines the $n$-alkanes generation from the kerogen and derived from different biological precursors and thermal cracking of hydrocarbons in the early diagenesis. The CPI values found in Tumaco-1-ST$\mathrm{P}$ well present a wide dispersion ranging between 0.47 and 


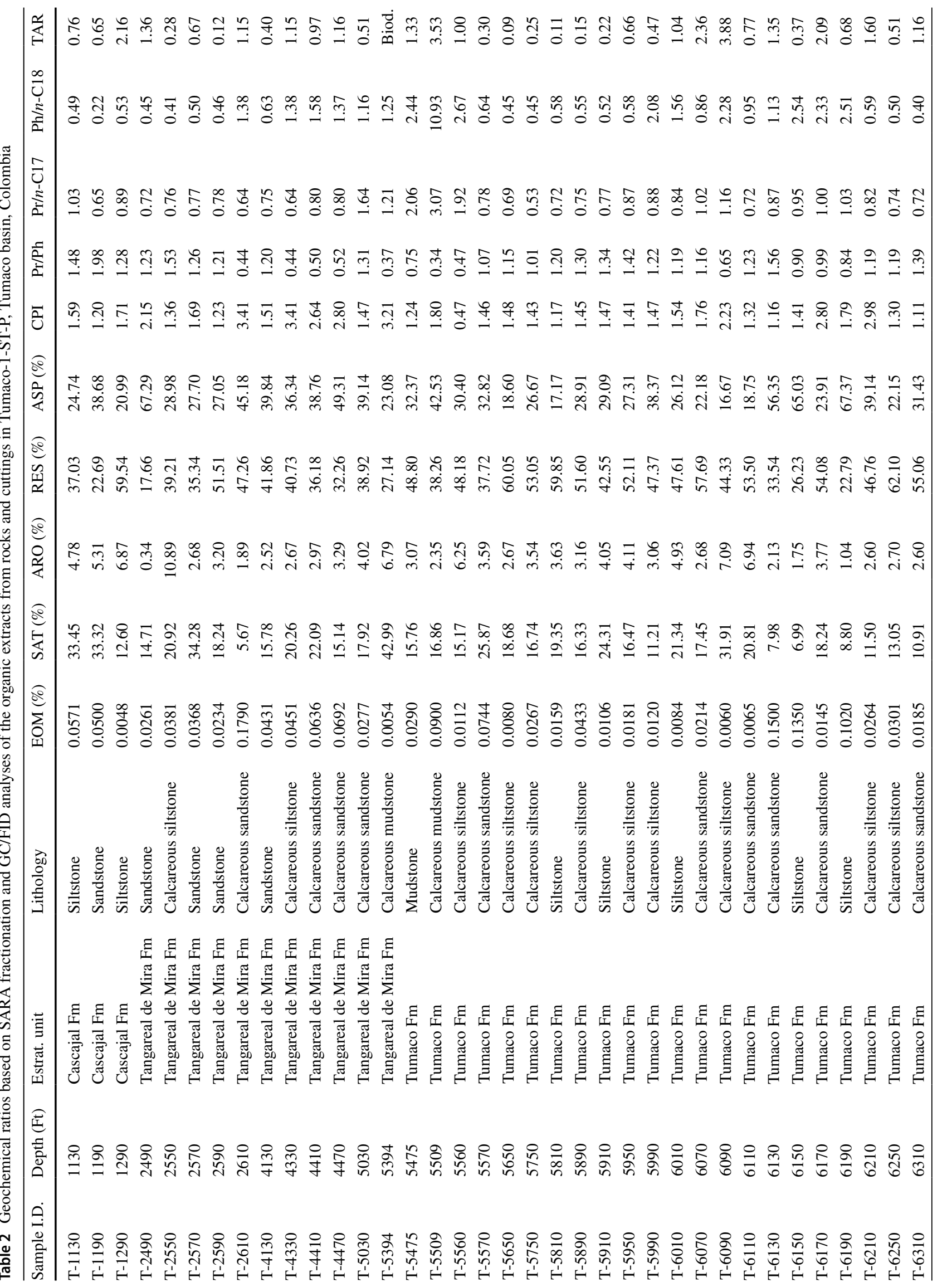




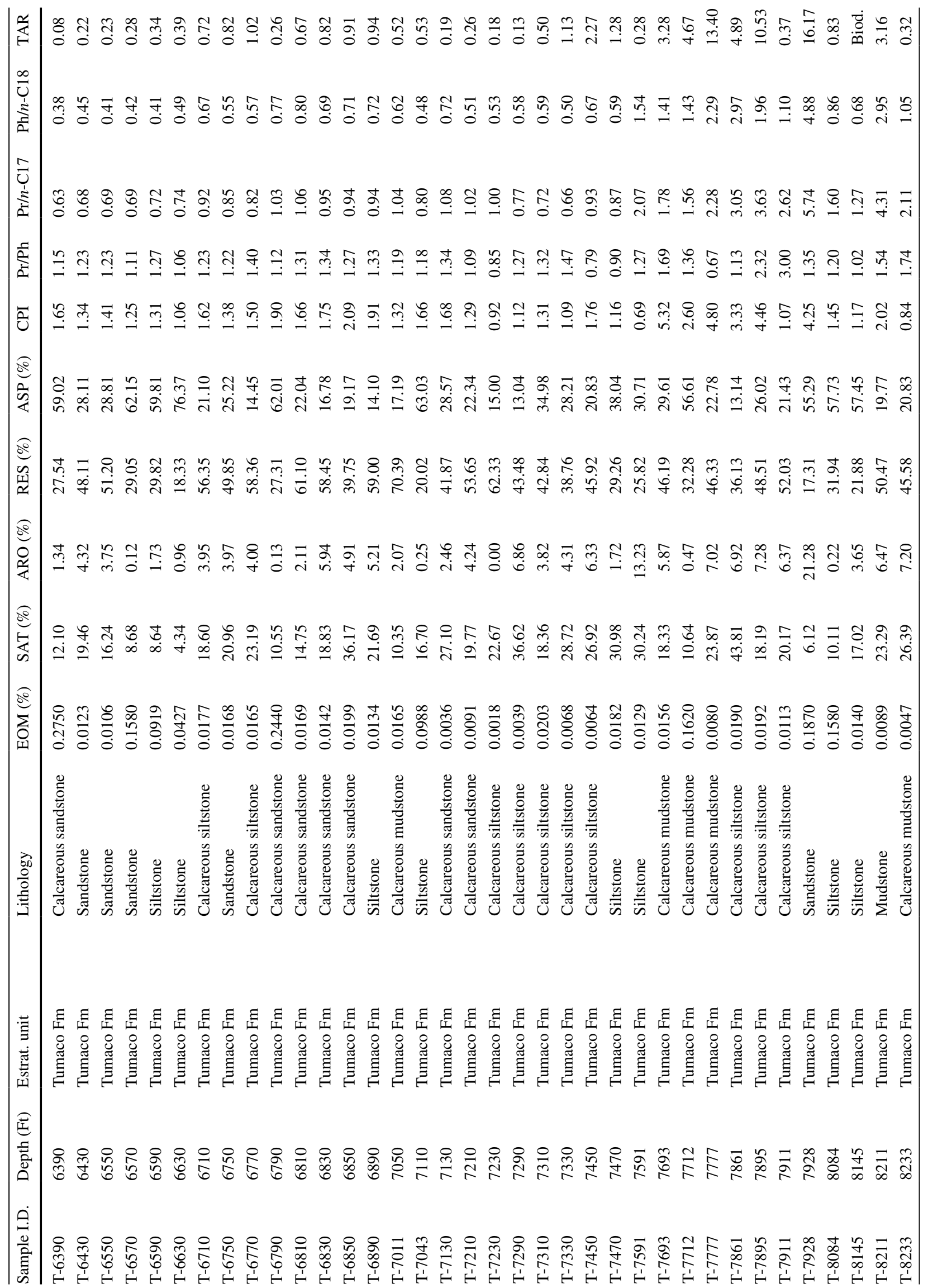




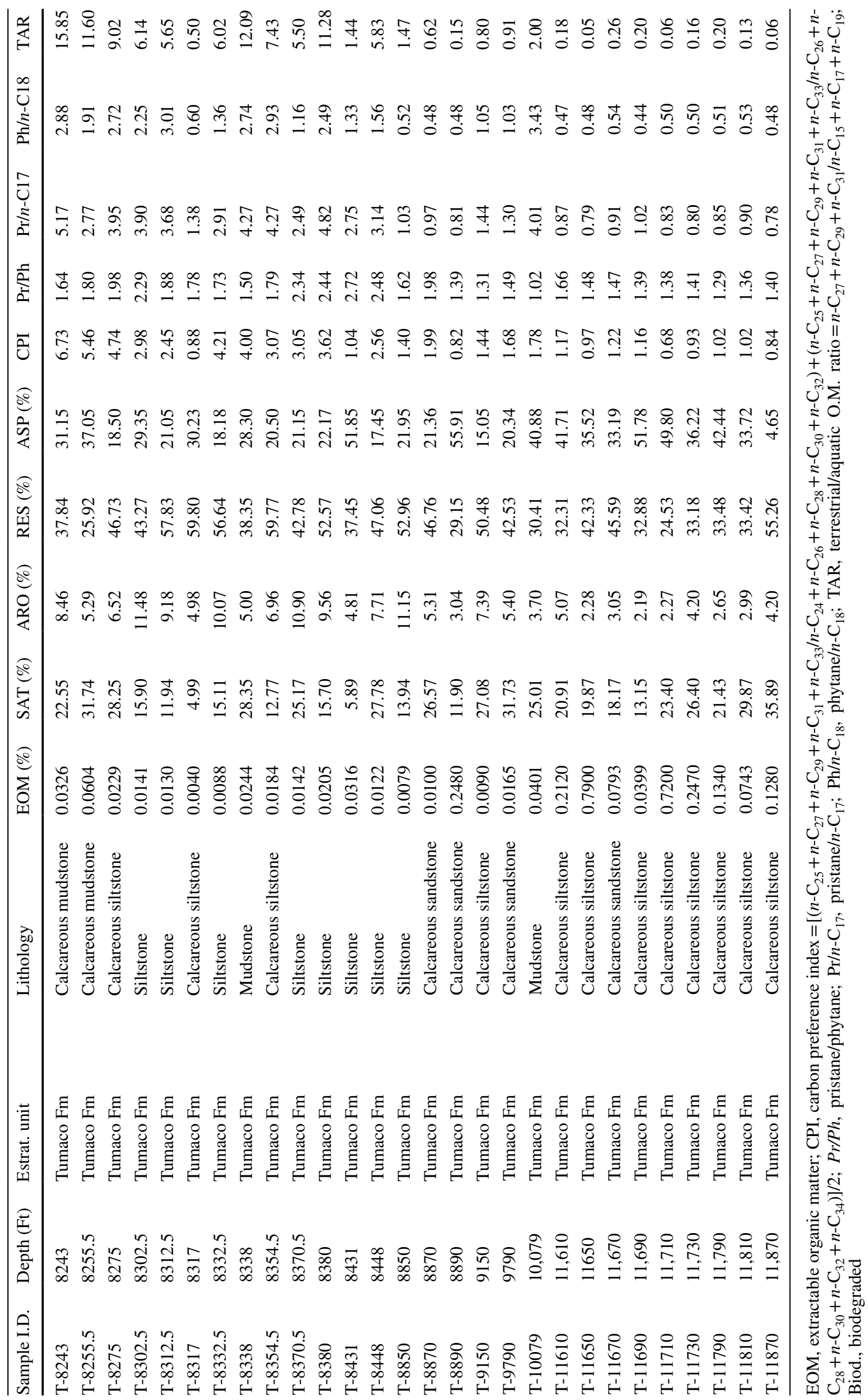


Fig. 11 Geochemical correlation between Isoprenoids $/ n$ alkanes showing the sources and depositional environment of the organic matter in the Tumaco-1-ST-P, Tumaco basin, Colombia

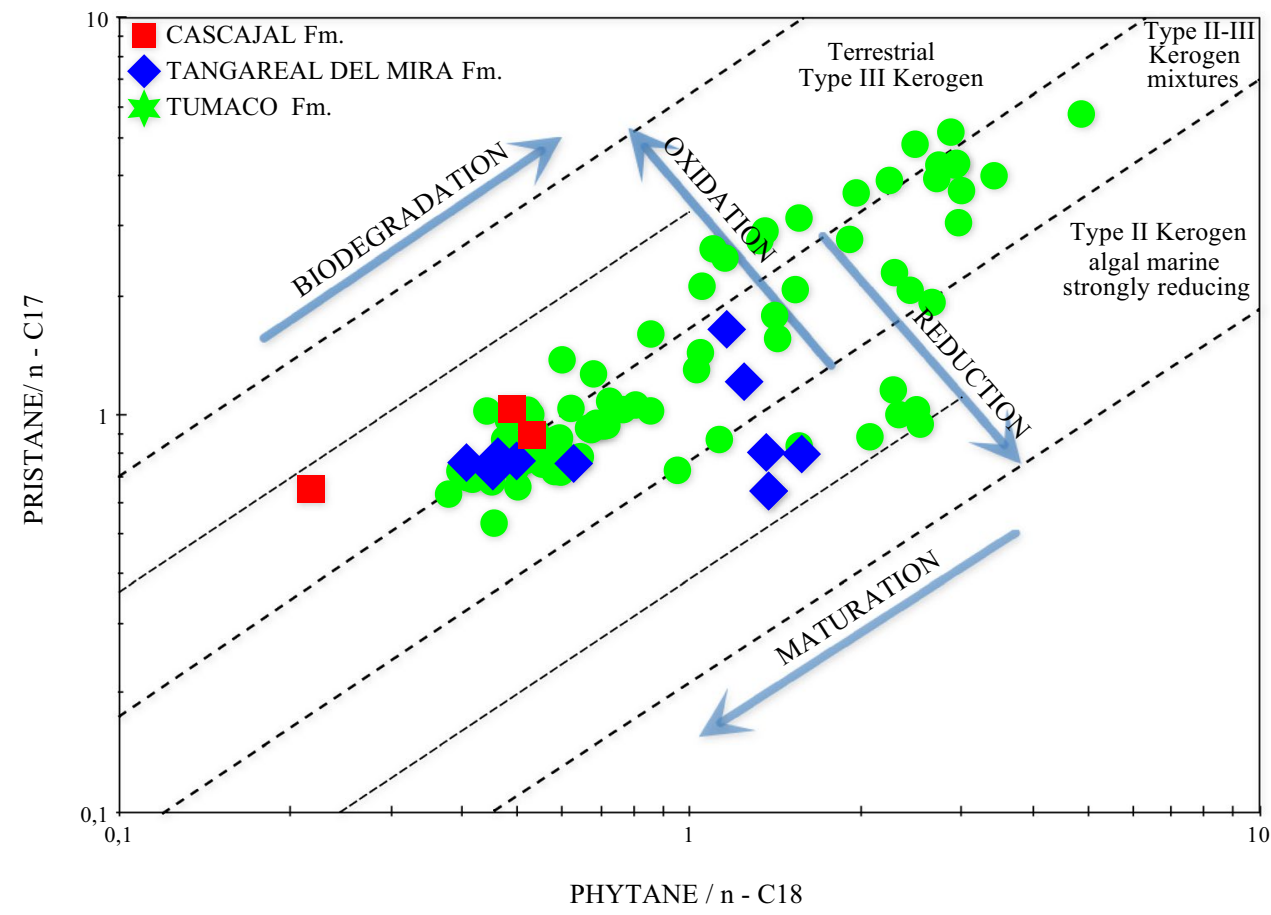

6.73, with a high amount of CPI values ranging between 1 and 3 and a few number of samples $(<6 \%)$ with $\mathrm{CPI}<1.0$. High values of CPI indicate low maturity and terrestrial origin, bitumen with CPI around 1 originating from marine contributions. Samples from Cascajal Fm present CPI values ranging between 1.20 and 1.71 indicating a marine-deltaic transitional O.M. source located in a paleoenvironment of deltaic plain highly immature. The Tangareal del Mira bitumen presents CPI in the range between 1.23 and 3.41 confirming a transitional marine-deltaic depositional environment, while the Tumaco Fm maintains the same marinedeltaic influence of the previous formation up to a depth of approximately $8000 \mathrm{ft}$, from which up to $12,000 \mathrm{ft}$, becomes a paleoenvironment with a tendency to the marine platform (shelf marine).

The vertical distribution of terrestrial/aquatic O.M. ratio (TAR) values is relevant to determine the relative changes in the contribution of the terrestrial versus aquatic O.M. over the time, particularly considering recent sediments (Meyers 1997). TAR displays values varying between 0.05 and 16.17 (average 2.18), which confirms the constant variations of organic facies from algal/bacterial and/or land origin through the stratigraphic column. High TAR values in recent sediment extracts, as in the case of Tumaco-1-ST-P, indicate a great contribution by land plants that aquatic to the depositary environment (Bourbonniere and Meyers 1996, cited by Peter et al. 2005). This ratio should be used with care since it is susceptible to drastic changes by effects of maturation and biodegradation.

\section{Terpanes and steranes}

The biological markers were identified based on full-scan GC/MS analysis, comparing the mass fragmentograms of the specific biomarkers with data previously reported (Philp 1985; Wang 1993; Peter et al. 2005) and with bitumen and crude oils of known composition (e.g., NGS NSO-1 oil sample, NPD-Nigoga; Boscan crude oil, Venezuela; Cauca/Patia and Buenaventura rock extracts, Colombia) (NPD-Nigoga 2000; Antek S.A. 2011).

Figure 12 (on the left) shows the tri-, tetra-, and pentacyclic terpane mass fragmentograms $(\mathrm{m} / \mathrm{z}$ 191) and steranes $(\mathrm{m} / \mathrm{z}$ 217) (on the right) for Tumaco-1-ST-P selected samples, while Table 3 displays the main geochemical correlation from terpane and sterane results. The terpane chromatographic profiles exhibit a particular features such as that the $\mathrm{C}_{30} \alpha \beta(\mathrm{H})$-hopane is the biggest terpane; the tricyclic terpane fraction extends from C19/3 until C30/3-R/S, Ts $<\mathrm{Tm}$; the oleanane and gammacerane are present in medium to high concentrations; homohopanes are present in relatively low concentration and in some samples do not exist. When they are present, are distributed between $\mathrm{C}_{31}$ and $\mathrm{C}_{35}$, decreasing in the order $\mathrm{C}_{31}>\mathrm{C}_{32}>\mathrm{C}_{33}>\mathrm{C}_{34}>\mathrm{C}_{35}-\mathrm{R} / \mathrm{S}$.

Geochemical correlations based on the tricyclic terpanes such as TT19/TT23, TT23/TT30, TT24/TT30 and TT25/ TT26 are excellent indicators of source and depositional environment. Thus, Table 3 shows that TT19/TT23 ratios vary between 0.06 and 0.58 , except for the sample T-1190 that presents a value of 1.10 . These low ratios suggest an important bacterial/algal over terrestrial origin. TT21/TT23 


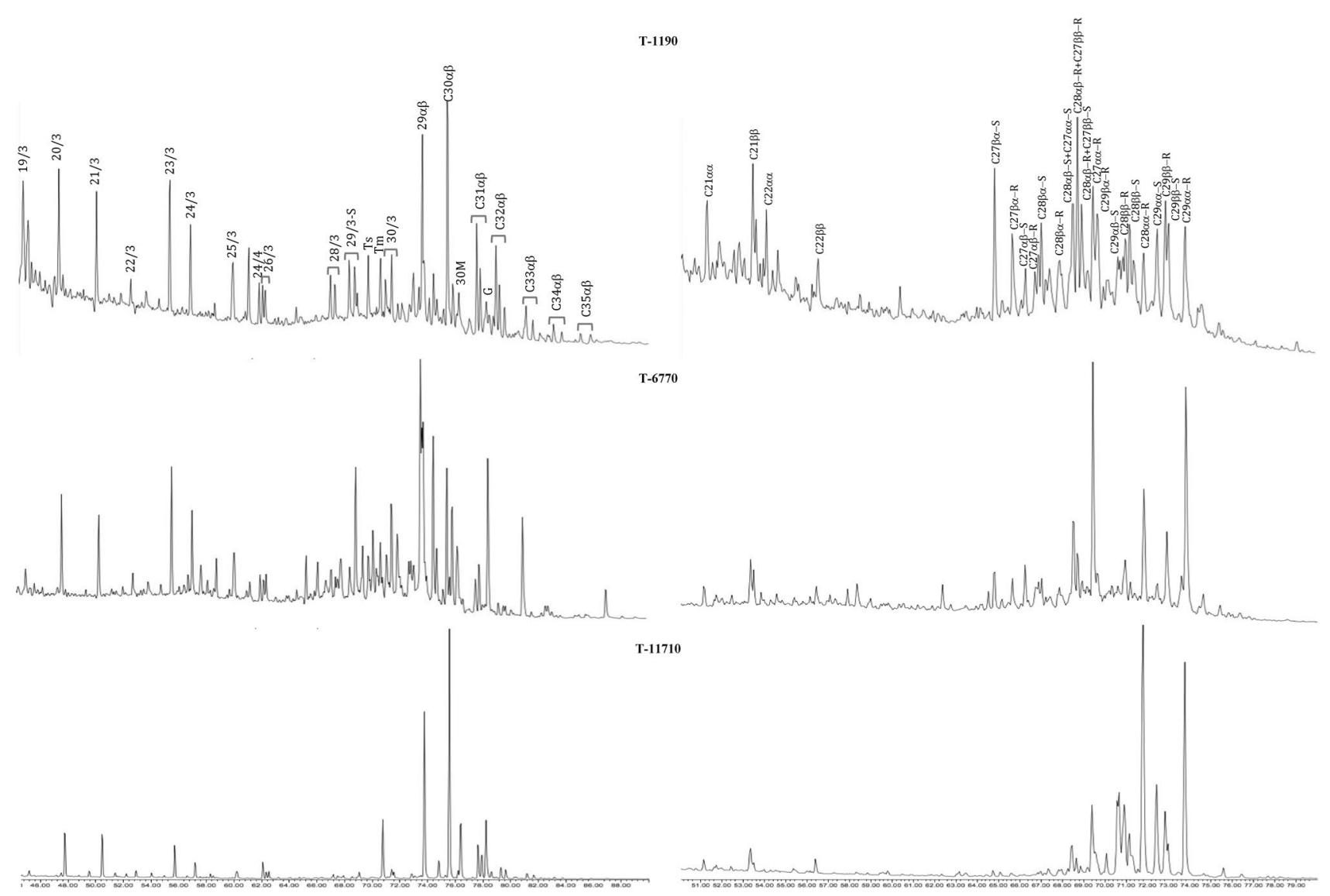

Fig. 12 Mass fragmentograms showing the terpane $(\mathrm{m} / \mathrm{z}$ 191) and sterane $(\mathrm{m} / \mathrm{z}$ 217) distribution in Tumaco-1-ST-P well, Tumaco basin, Colombia

ratios present values $>1.0$ for most of the samples corroborating a marine origin associated with prokaryotic organisms (algal/bacterial). However, in the shallower intervals of the Cascajal and Tangareal del Mira Fm, the TT21/TT23 ratios display values slightly higher than 1 , indicating a combined contribution of bacterial/algal O.M. with a significant terrestrial input.

TT23/30H ratios and $\Sigma$ tricyclic terpanes/ $\Sigma$ hopanes do not show a trend that clearly shows the origin of the O.M. along the stratigraphic column. Values vary alternately around 1.0, indicating a significant higher plant (continental) contribution to the O.M. toward the shallower formation of Cascajal and Tangareal del Mira, while in the deepest part of the well, the Tumaco Fm, the O.M. is mainly marine contributions from bacterial/algal inputs. C24TeT/C30-hopane ratios are quite used in the characterization of the paleodepositional environment (Peter et al. 2005). The highest abundances of tetracyclic terpane C24TeT have been found in marine-deltaic and lacustrine saline environments (Rocha-Mello et al. 1988). In Tumaco-1-ST-P, C24Te/H30 ratios present values $<0.44$, which dismisses a carbonate or evaporitic environment but it supports a marine shale or marine-deltaic depositional environment. Burwood et al. (1990) found that marine bitumen and crude oils from West Africa showed TT25/TT26 ratios $>1.0$, while terrestrial bitumen showed TT25/TT26 values $<1.0$. Thus, in the Tumaco-1-ST-P well, this ratio confirms the marine origin of the studied samples as is shown in Fig. 13. Since TT24/TT23 is greater than 0.5 for most of the samples in Tumaco-1-ST-P suggest organic facies associated with marine shales.

Steranes in selected samples from Tumaco-1-ST-P (Fig. 12) allow observing that pregnanes, excellent indicators of salinity, are present in medium concentration, although in some samples these are biodegraded or have not been formed. The $\mathrm{C}_{27}$ diasteranes (rearranged steranes) are present in significant concentrations, which suggest a silisiclastic environment (27D/29 $\alpha \alpha \mathrm{R}>1.0)$. C27-, C28-, and $\mathrm{C} 29$-steranes are present in varying concentrations and without any predominance between them, which suggest alternating contributions of algal/microbial and land plants (transitional environment) origin. The configuration of $\alpha \alpha \mathrm{R}$ sterane in most of the samples is greater than $\beta \beta$-R-S or $\beta \beta-\mathrm{S}$, which indicates an immature O.M.

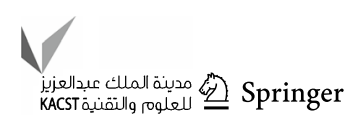




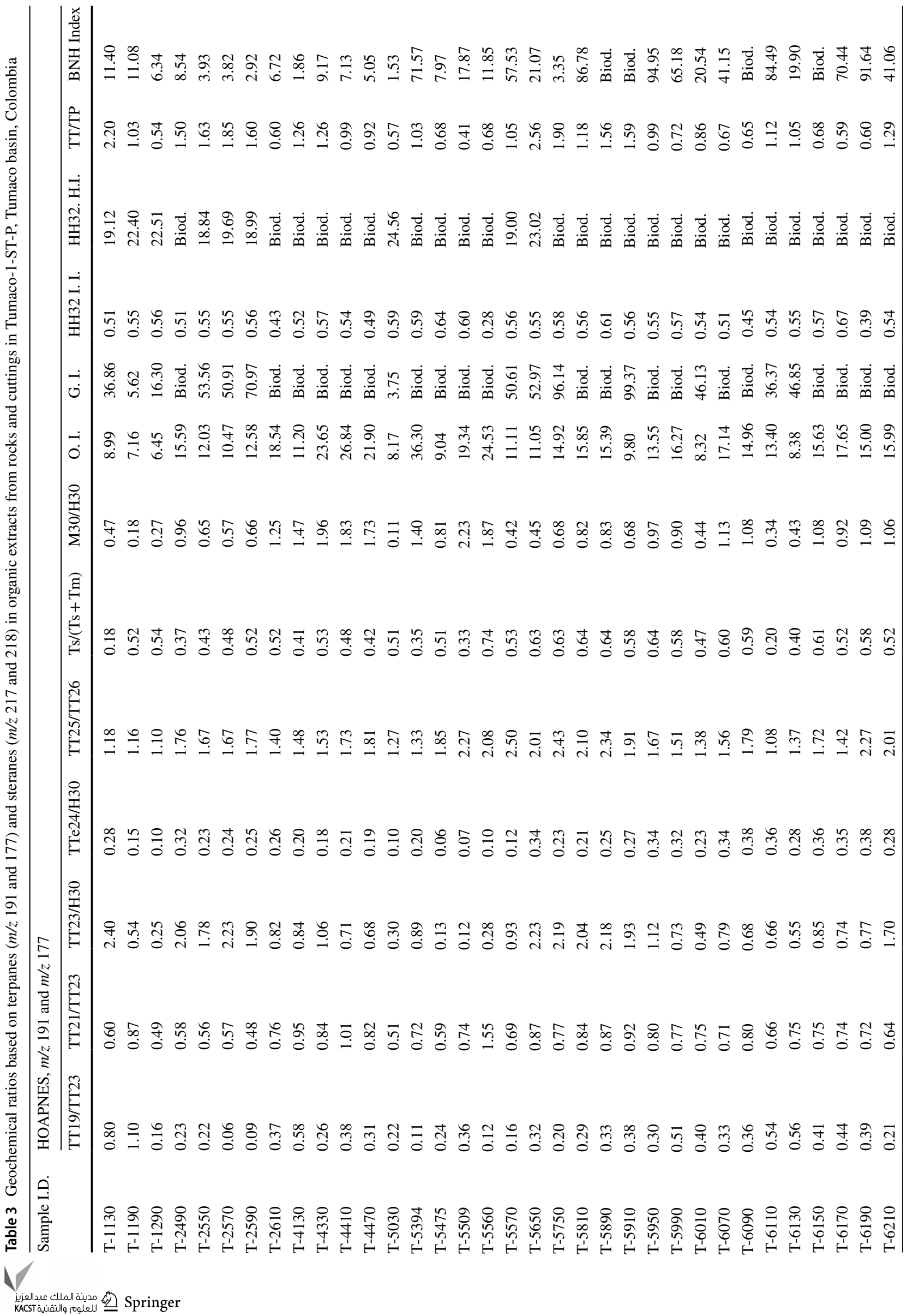




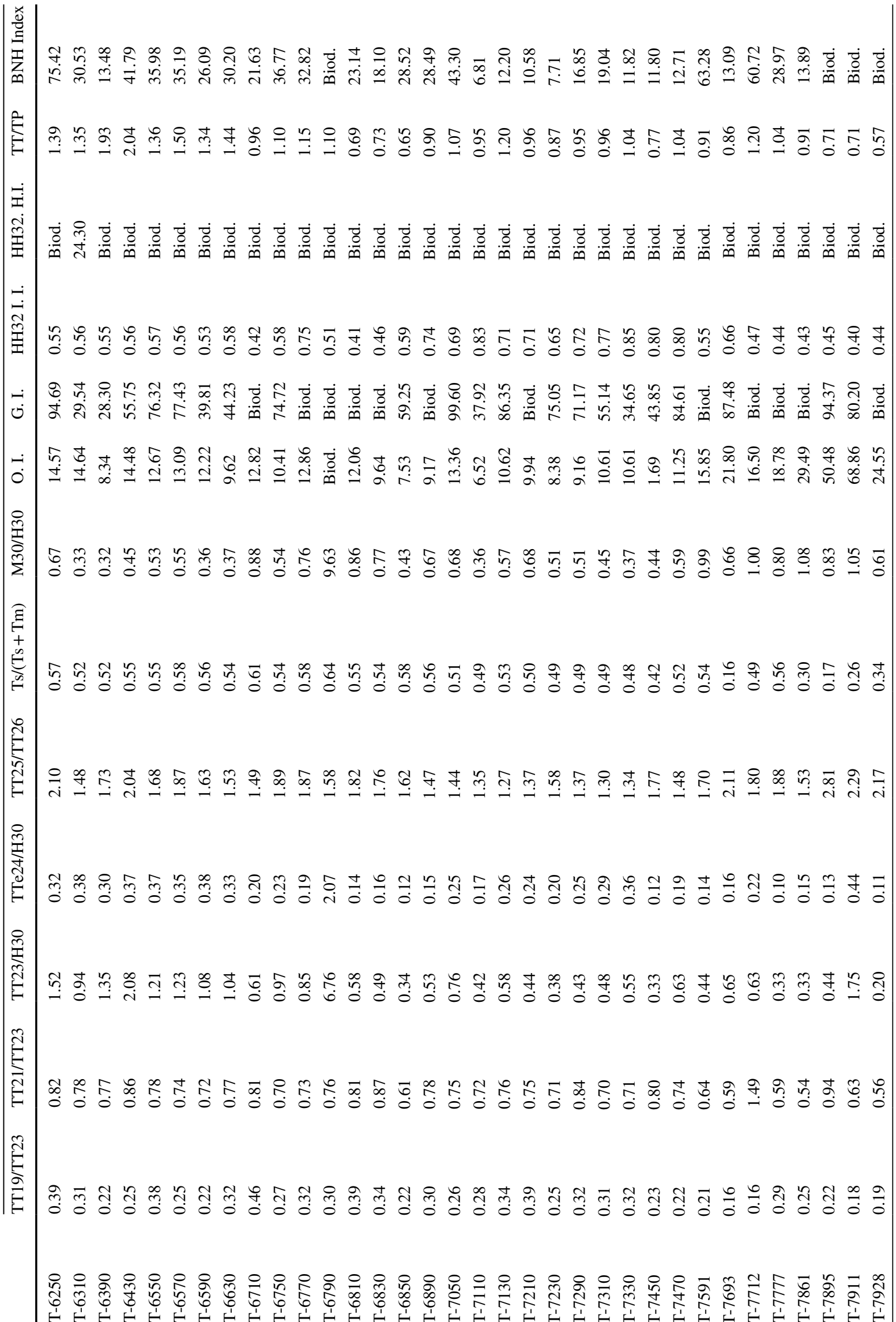

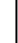




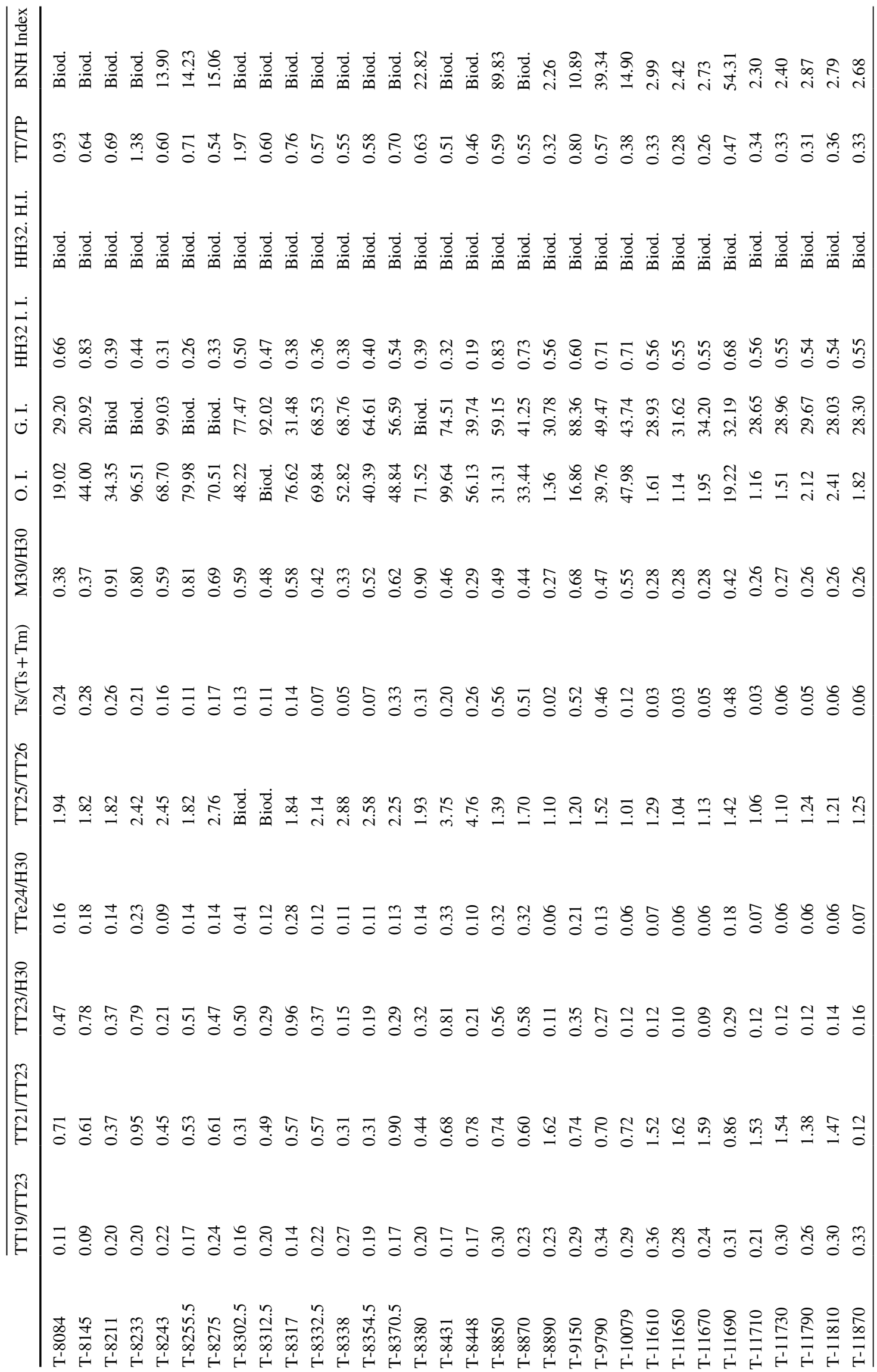




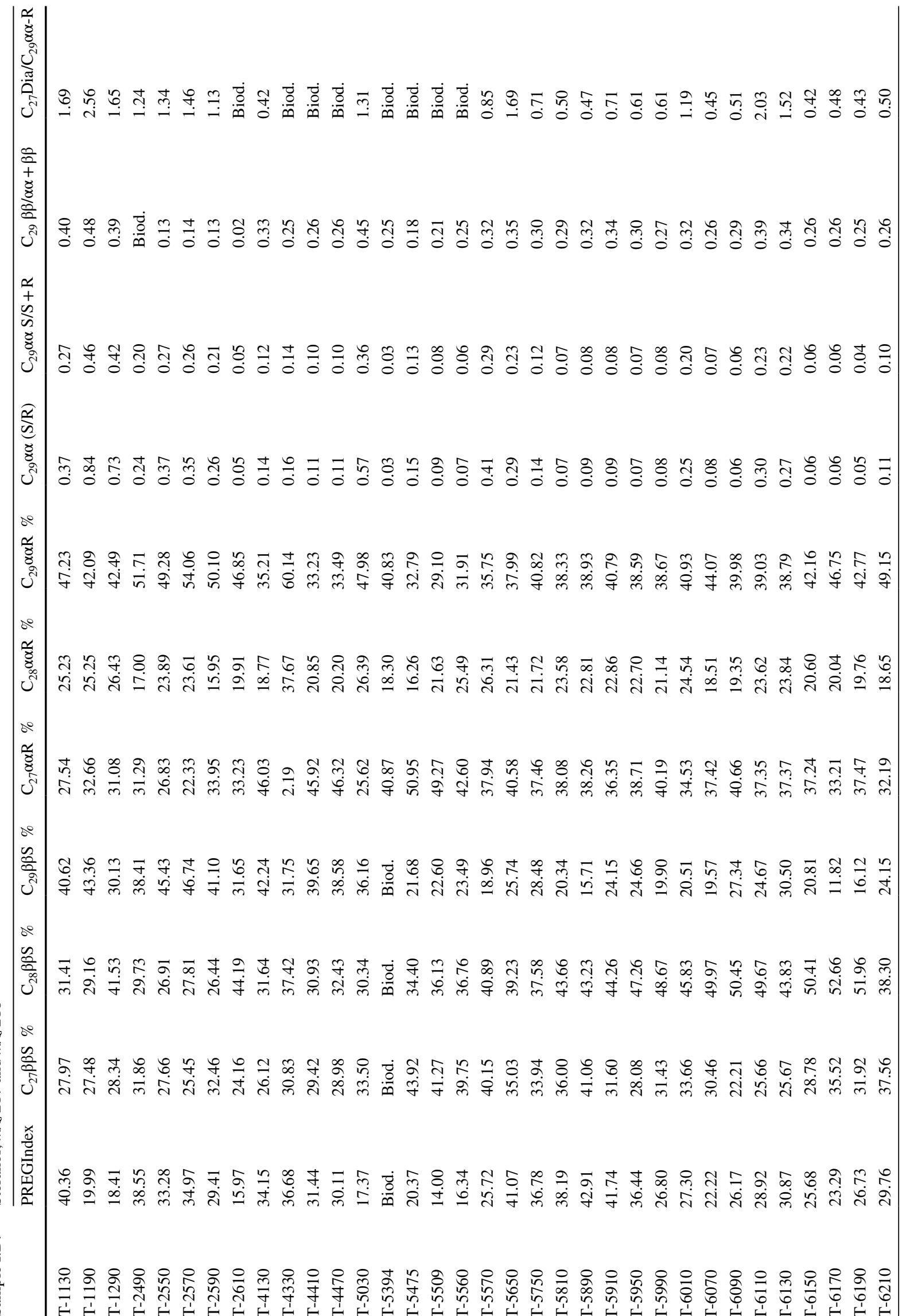




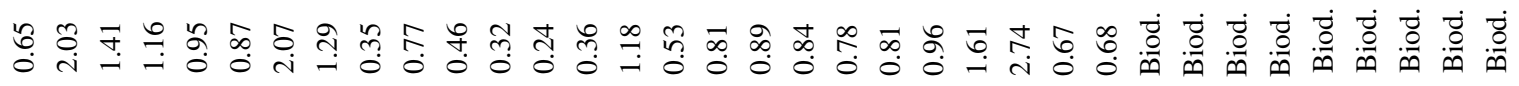

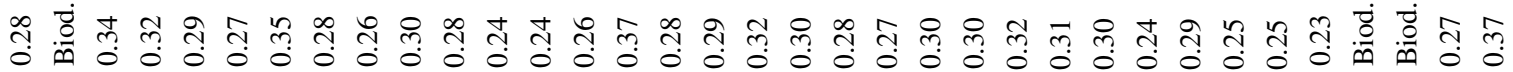

$\frac{1}{4}$

然

今.

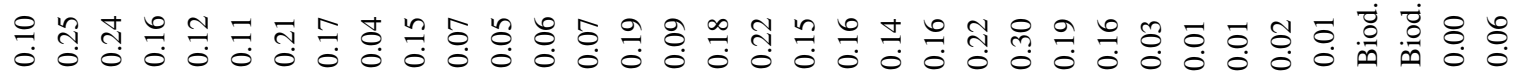

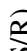

敢

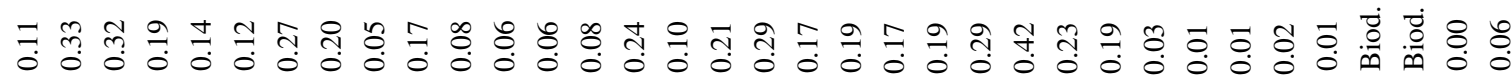

$\therefore$

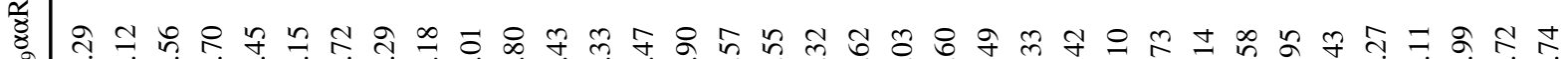

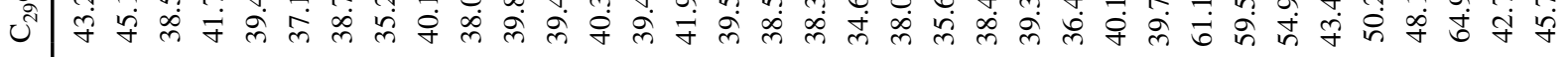

\& to बें

\&

\&

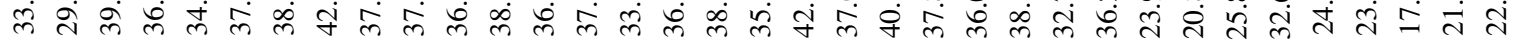

so

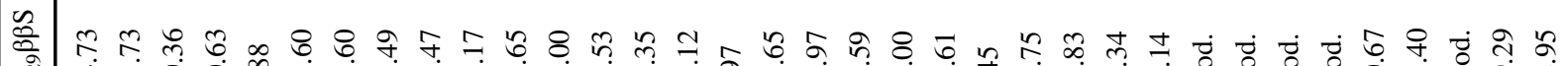
过

se

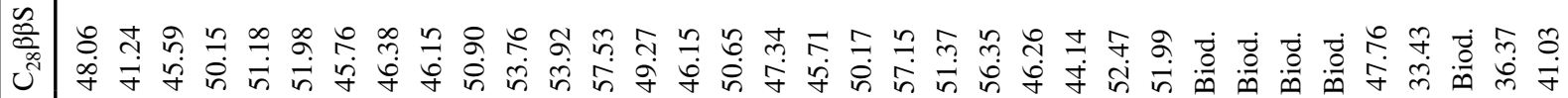
$\stackrel{\infty}{\sim}$

त्र

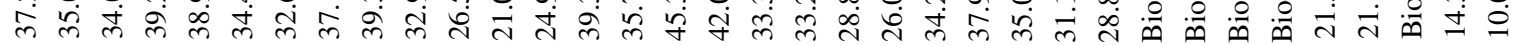

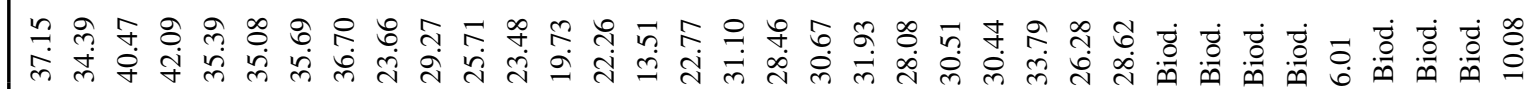

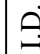
$\begin{array}{ll}0 & \\ 0 & 0\end{array}$ 


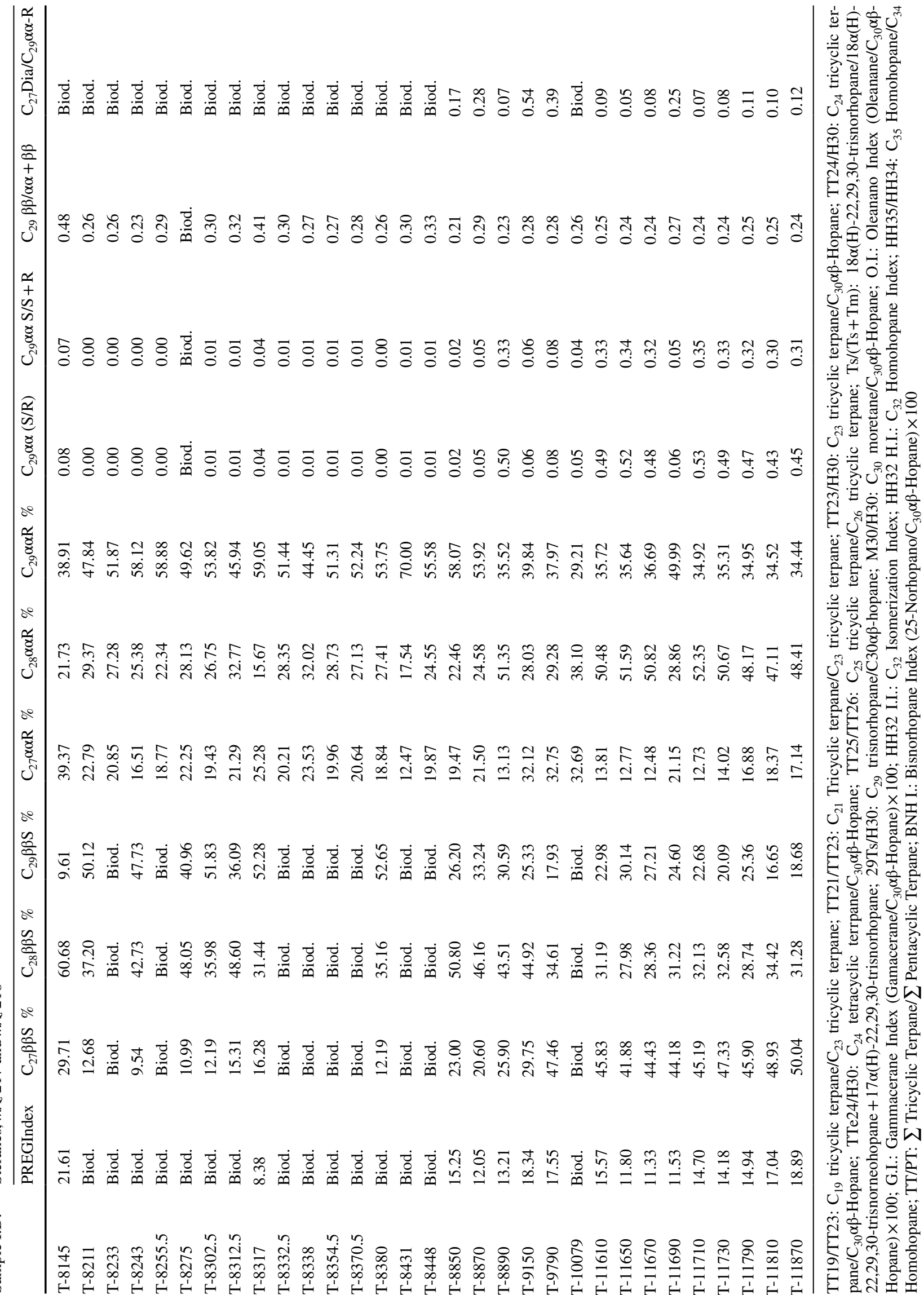


Fig. 13 Geochemical correlation between $26 / 25$ tricyclic terpanes versus $24 / 23$ tricyclic terpanes in the organic extracts from rock in Tumaco-1-ST-P, Tumaco basin, Colombia

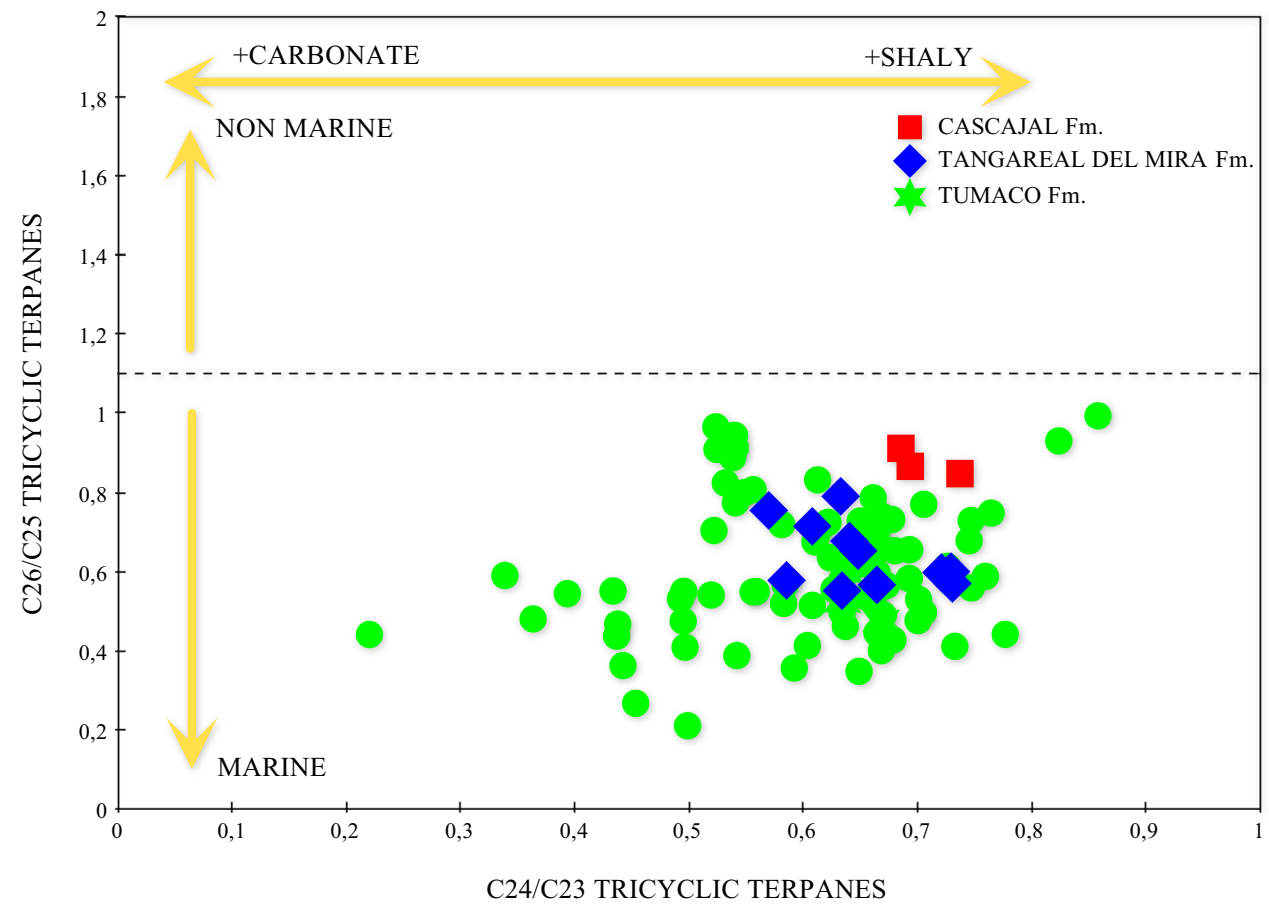

The diasteranes/steranes ratio is routinely used in geochemical studies to distinguish between carbonate and siliciclastic source rocks. Low values of this ratio in bituminous extracts indicate anoxic carbonate source rocks poor in clays. On the contrary, high diasteranes/esteranes ratios are typical of bitumen derived from source rocks containing abundant clays (Cortes et al. 2018). The diasterane/sterane (27-dia/29 $\alpha \alpha \mathrm{R})$ ratios for Tumaco-1-ST-P well show values $>2.0$, which clearly indicates a higher diasteranes content over the regular steranes, suggesting that this organic matter was deposited on siliciclastic rocks in a transitional marine-continental environment.

The $20 \mathrm{~S} /(20 \mathrm{~S}+20 \mathrm{R})$ ratio is one of the maturity parameters more frequently used in petroleum geochemistry. The isomerization of carbon $\mathrm{C} 20$ on the $\mathrm{C} 29$ $5 \alpha(\mathrm{H}), 14 \alpha(\mathrm{H}), 17 \alpha(\mathrm{H})$-sterane causes an increase in the $20 \mathrm{~S} /(20 \mathrm{~S} 20 \mathrm{R})$ ratio between 0.0 to $\approx 0.5$ with an increment of the maturity (equilibrium between 0.52 and 0.55 , Seifert and Moldowan 1986) (Cortes et al. 2018). Bitumen in Tumaco-1-ST-P well has values in the range between 0.0 and 0.4 (average 0.13 ), which indicates a very low maturity. The C29 $\beta \beta /(\beta \beta+\alpha \alpha)$ ratio typically presents its thermal equilibrium between 0.68 and 0.72 . Bitumen extracts from Tumaco-1-ST-P rocks show a value around 0.1, confirming that these samples are immature or in the diagenetic stage.

\section{Maturity evolution}

\section{Aromatic hydrocarbons}

Thermal maturity is the most important process in evaluation of oil and gas potential. Geochemical correlations based on aromatic hydrocarbons can be potentially better than those obtained in the saturated biomarkers fraction due to they are sensitive to differences in thermal maturity from the middle stage to the final part of the window of hydrocarbons generation (Radke 1988). A great variety of geochemical ratios based on the composition of the aromatic fraction has been proposed associated with the relationship between the concentration of the more thermally stable isomers and their counterparts less stable (Radke and Welte 1983; van Aarssen and de Leeuw 1999).

Maturity parameters derived from methylnaphthalenes $(\mathrm{MN})$ are based on the idea that naphthalenes with compound methyl $\beta$-substituted are more stable than those with methyl $\alpha$-substituted. The maturity parameters based on the phenanthrene (P) and methylphenanthrenes (MP) are based on the high stability of the 3-MP and 2-MP compared with 9-MP and 1-MP (Radke and Welte 1983). The isomers of the alkyldibenzothiophenes (MDBT) with a methyl substituent in position 4 are more thermodynamically stable, whereas 
isomers with a methyl substituent in position 1, have low stability (Chakhmakhchev et al. 1997).

In Tumaco-1-ST-P bitumen extracts, the aromatic hydrocarbons were identified based on full-scan GC/MS analysis and quantified by GC/MS-SIM mode using the selected ions for alkylnaphthalenes, alkylphenanthrenes, alkyldibenzothiophenes, mono- and triaromatic steroids (Sect. 3.6) comparing the mass fragmentograms with data previously reported (Hughes 1984; Budzinski et al. 1995; van Aarssen and de Leeuw 1999) and with bitumen and crude oils of known composition (e.g., NGS NSO-1 oil sample, NPDNigoga) (NPD-Nigoga 2000).

Figure 14 shows the alkylnaphthalenes, alkylphenanthrenes, and alkyldibenzothiophenes fragmentograms for sample T-11610 of Tumaco-1-ST-P well, while Table 4 displays the main geochemical correlation with aromatic results. There is evidence that in the hydrocarbon generation window, the aromatic hydrocarbons show systematic changes with an increment in the thermal maturity (Hughes 1984; Radke and Welte 1983; Alexander et al. 1985). However, in an early stage (diagenesis), the geochemical parameters based on the alkylaromatics, as in Tumaco-1-ST-P, show an irregular behavior due to quick variations in the organic facies, control from source effects, thermal stress, and biodegradation of the O.M.

Methylnaphthalenes are found and widely distributed in the sedimentary organic matter (Tissot and Welte 1984). Their distribution is highly variable among samples (even in the same producer field or in a stratigraphic column) (van Aarssen and de Leeuw 1999). The sesquiterpanes and triterpenoids derived from microorganisms and superior plants appear to be the origin of the MN (Püttman and Villar 1987), where they are produced through isomerization and transmethylation reaction in an environmental rich in acid clays. In Tumaco-1-ST-P, MN ratios vary between 0.25 and 5.19 (mean 1.68 \pm 0.73 ) indicating a high variability in the maturity and suggesting a common origin but different states of thermal maturity consistent with an intense diagenetic biodegradation process. As the thermal maturity increases, the predominance of 2-MN over 1-MN increases. This increment toward the depth must be interpreted as an increase in the maturity toward deeper intervals (Tumaco Fm, late Oligocene). The dimethylnaphthalenes ratios (DNR) vary between 0.03 and 21.5 indicating a wide maturity distribution. Among TMNs, the most abundant isomer for a mixture in equilibrium (highly mature) is the 2,3,6-TMN, and one of the less abundant is the 1,2,5-TMN, which is often the most abundant in immature samples (Budzinski et al. 1995). van Aarssen and de Leeuw (1999) found that the presence of 2,3,6-TMN is very low in the diagenetic stage and increases dramatically in samples highly mature in which this isomer is dominant over the 1,3,7-TMN and 1,3,6-TMN.
The distribution of alkylphenanthrenes can be used as an indicator of source, in addition to its well known use as a parameter of maturity (Armstroff et al. 2006). Differences due to variations in the thermal maturity are in many cases gradual, while changes in the source of the O.M. are characterized by drastic differences. The alkylphenanthrenes distribution is dominated by 2-MP and 9-MP isomers at any stage of maturity. In Tumaco-1-ST-P well, 2-MP and 9-MP are predominant over 1-MP and 3-MP indicating a marine origin (Budzinski et al. 1995).

Methylphenanthrene-1 and -2 indices (MP-1 and MP-2) proposed by Radke and Welte (1983) are perhaps the most widely used indices to determine the maturity based on aromatic hydrocarbons. The utility of this geochemical parameter rests on the relative stability of the various methylphenanthrene isomers. Due to its linear relationship with the vitrinitic reflectance within the generation window, MPI-1 is often used to infer the value of the equivalent vitrinitic reflectance (Rc, \%) in crude oils (Radke 1988) and could be used in source rocks and bituminous extracts of outcrops where the vitrinitic reflectance cannot be measured. The MPI-1 values in Tumaco-1-ST-P show an average value of 0.60 and a mean value of $\mathrm{Rc}$ of $0.76 \%$.

Since 4-methyldibenzothiophene isomer is more stable than 1-methyldibenzothiophene, the 4-/1-methyldibenzothiophene (MDBTR) was proposed as an indicator of maturity (Radke et al. 1986). This ratio is quite sensitive to the maturity, correlating well with the vitrinitic reflectance and with $T_{\max }$ parameter from pyrolysis Rock-Eval in rock samples (Radke 1988). Dzou and Hughes (1993) noted that MDBTR is particularly robust to assess maturity in samples with high thermal maturity (condensates, lighter crudes). The organic extracts of the Tumaco-1-ST-P well presented an average value of 5.40, indicating that the 4-methyldibenzothiophene is in the highest concentration that 1-methyldibenzothiophene. The maturity based on MDBT ratios increases from surface to bottom in the column reaching the highest values in the Tumaco Fm between the early and middle Miocene.

The dibenzothiophene/phenanthrene ratio (DBT/P) is thought to be an indicator of source rock lithology (Radke et al. 1986, Hughes et al. 1995) with carbonates having ratios $>1$ and shales $<1$. When this ratio is plotted against $\mathrm{Pr} / \mathrm{Ph}$, the depositional paleoenvironment may be inferred regarding microbiological and chemical processes (Hughes et al. 1995). Figure 15 shows a plot of DBT/P versus $\mathrm{Pr} / \mathrm{Ph}$ associated with rock lithology for a bituminous extract of Tumaco-1-ST-P, indicating that samples fall in the area of a lacustrine and marine shale environment (zones 2 and 3, respectively). The zone 2 suggests a variable lithology of the rock with values of DBT/P $<1$ and values of $\mathrm{Pr} / \mathrm{Ph}<1$ and characterizing an lacustrine environment, while the zone 3

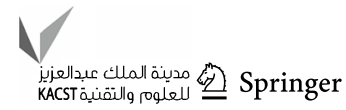




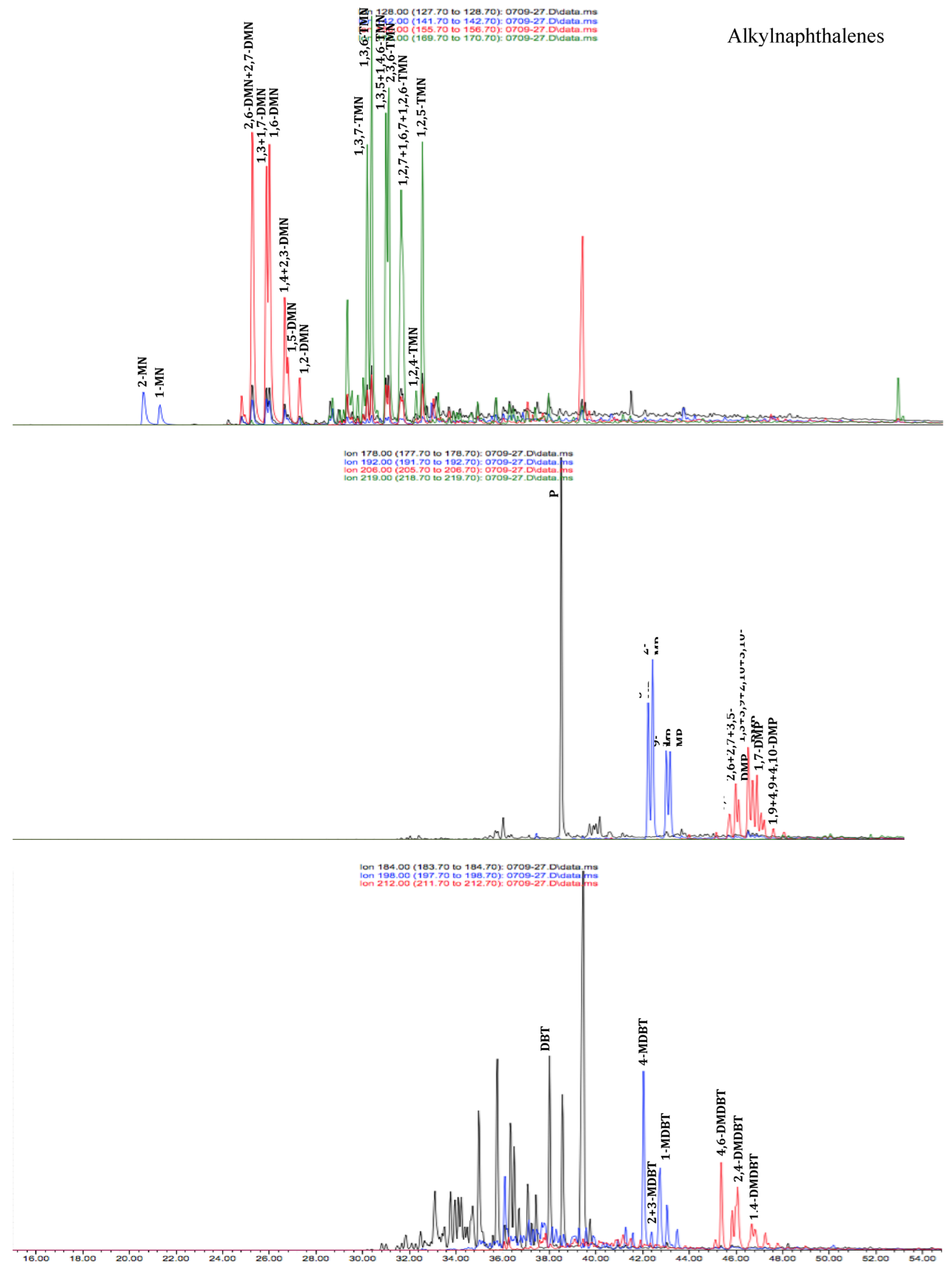


४Fig. 14 Alkylnaphthalenes, alkylphenanthrenes, dibenzothiophene and alkyldibenzothiophenes de la muestra T-11610, Tumaco-1-ST-P, Tumaco basin, Colombia (see foot Table 4 for compound identification)

presents a lithology of shale, in an marine environment and $\mathrm{DBT} / \mathrm{P}<1$ and $\mathrm{Pr} / \mathrm{Ph}$ in the range between 1 and 3.

\section{Molecular stratigraphy in Tumaco-1-ST-P}

The molecular stratigraphic interpretation allows to conclude that organic facies and lithology in Tumaco-1-ST-P are characterized in Cascajal and Tangareal del Mira Fms. $(1000-5000 \mathrm{ft})$ by a marine-deltaic paleoenvironment with an important terrestrial contribution and varying between deltaic plain and prodelta during the late Miocene as is supported by $\mathrm{C}_{15}{ }^{+} n$-alkanes profiles, CPI, $\mathrm{Pr} / \mathrm{Ph}$, TAR, oleanane index, and tricyclic/pentacyclic terpanes (Internal communication stratigraphy Antek S.A.-Paleoxplorer in ANH-Antek S.A. 2013). At the top of Tumaco Fm (5000-9000 ft) in the late to medium Miocene, the depositional environment was like to the marine delta of the Tangareal del Mira Fm, changing to a marine platform environment at 8000 to $10,000 \mathrm{ft}$ depth in the middle to early Miocene, which is confirmed by high $\sum$ tricyclic terpanes/ $\sum$ pentacyclic terpanes, low oleanane index, high C35/C34 homohopanes ratios. Toward the bottom of the column $(10,000-12,000 \mathrm{ft})$, Tumaco Fm was deposited in the late Oligocene under a marine shelf environment, which is supported by $\mathrm{Pr} / \mathrm{Ph}<1.0, \mathrm{DBT} / \mathrm{P}<1.0$, low C26/C25 tricyclic terpanes ratios, high C24/C23 tricyclic terpanes ratios.

\section{Conclusions}

Total organic carbon in samples from Tumaco-1-ST-P presented an average value of $0.95 \%$ on a population of 250 samples analyzed, with a variation between 0.09 and $2.30 \%$. This variability suggests a temporal and spatial variations of organofacies in the sedimentary record. Results of Rock-Eval pyrolysis showed that $\mathrm{S} 1$ peak varies between 0.02 and $0.80 \mathrm{mg} \mathrm{HC} / \mathrm{g}$ rock, which classifies the O.M. with a "poor to acceptable" generation potential. S2 peak varied between 0.07 and $10.45 \mathrm{mg} \mathrm{HC/g}$ rock (average $1098 \pm 1.36 \mathrm{mg} \mathrm{HC} / \mathrm{g}$ rock). Peak S3 showed values between 0.20 and $7.60 \mathrm{mg} \mathrm{CO} 2 / \mathrm{g}$ rock, while $T_{\max }$ values varied in the range between 328 and $445^{\circ} \mathrm{C}$, which indicates a relative immaturity of the O.M. (diagenetic maturity).

Geochemical correlations based on screening data allowed to conclude that $\mathrm{S} 2 / \mathrm{S} 3$ varied in the range between 0 and 10 , whereas $\mathrm{HI}_{\mathrm{pd}}$ presented three major peaks that classified the O.M. quality with a type III and/or type II/ III kerogen. This classification would expect that the main product generated will be crude oil and/or crude oil/gas, special in the deepest intervals of the well. Production index varied between 0.02 and 0.36 classifying these rocks in a immature generation stage. However, the combination of the I.P with other maturity parameters allowing to look, in a more discreet way, that the column covers a wide range of maturity from a kerogen oil-gas-prone type II/III to a kerogen non-generator type IV, indicating that the O.M. in the study does not come from a single and unique kerogen due to the large variation in paleodeposicional environments. On the basis of the modified van Krevelen diagram, the type III kerogen, which is responsible for the majority of immature samples from Tumaco-1-ST-P well was characterized to have low H.I (50-200 mg HC/g TOC), low H/C atomic ratios (0.7-1.0), and high $O / C$ values $(>0.3)$.

Vitrinite reflectance (Ro) varied between 0.1978 and $0.5418 \%$ (mean $0.4046 \pm 0.056 \%$ ), suggesting an immature O.M. The maceral groups found were vitrinite, liptinite, and inertinite, which allowed us to identify the following types of macerals: esporinite, cutinite, resinite, alginite, colotelinite, colodetrinite, funginite, exsudinite, exsudatinite, inertodetrinite, among others. The EOM, \% oscillation between $0.0018 \%$ and $0.75 \%$, being the highest contents toward the late Oligocene in the Tumaco Fm.

SARA fractionation presented high values for the resin fraction in most of the organic extracts, varying between 17.47 and $62.33 \%$. The asphaltenes varied in a range between 4.65 and $59.02 \%$, the aromatic fraction ranging between 0 and $21.28 \%$ is the fraction of which were obtained lower recoveries. Finally, saturated fraction ranged between 4.99 and $43.81 \%$. The Tissot and Welte's ternary diagram indicates that the organic matter extracted was of naphthenic characteristics.

$\mathrm{C}_{15}{ }^{+} \mathrm{GC} / \mathrm{FID}$ analysis of the saturated fraction showed a bimodal distribution in hydrocarbons resolved between $n$ $\mathrm{C}_{15}$ and $n-\mathrm{C}_{32}$, although some chromatograms extend their distribution to $n$-C 36 , with a maximum around $n-\mathrm{C}_{19}$ to $n$ $\mathrm{C}_{20}$ suggesting an algal/bacterial input, while the second peak occurs around $n-\mathrm{C}_{29}$ to $n$ - $\mathrm{C}_{31}$, indicating a continental contribution (terrestrial), allowing to conclude that this is a mixed O.M. originated from type II/III kerogen. The geochemical correlations based on $\mathrm{C}_{15}{ }^{+}$saturated fraction show a CPI characteristic of a low maturity and marine/continental origin. $\mathrm{Pr} / \mathrm{Ph}$ ratio suggests a bitumen derived from marine shale, additionally $\mathrm{Pr} / \mathrm{Ph}<3.0$, indicating that O.M. was deposited in suboxic to oxic conditions. $\mathrm{Pr} / n-\mathrm{C}_{17}$ varied between 0.53 and 5.74, while $\mathrm{Ph} / n-\mathrm{C}_{18}$ ranges between 0.22 and 4.88 , indicating low maturity. TAR values suggested that O.M. has an important aquatic algal/bacterial input over terrestrial contributions.

Geochemical correlations based on saturated fraction biomarkers analyzed by GCMS showed that TT21/TT23 present values $>1.0$ for most of the samples corroborating the

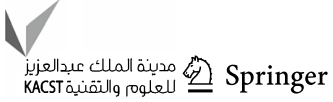


Table 4 Geochemical ratios based on Aromatic Hydrocarbons in organic extracts from rocks and cuttings in Tumaco-1-ST-P, Tumaco basin, Colombia

\begin{tabular}{|c|c|c|c|c|c|c|c|c|c|c|c|}
\hline \multirow[t]{2}{*}{ Sample } & \multicolumn{3}{|c|}{ Alkylnaphathalenes } & \multicolumn{2}{|c|}{$\begin{array}{l}\text { Alkylphenan- } \\
\text { threnes }\end{array}$} & \multicolumn{3}{|c|}{ Alkylbenzothiophenes } & \multicolumn{3}{|c|}{ Monoaromatic and triaromatic steroids } \\
\hline & MNR & DNR & TMNR & MPR & MPI-1 & DBR & MDBTR & $\mathrm{DBT} / \mathrm{P}$ & $\begin{array}{l}\text { TAS/ } \\
\text { (MAS + TAS) }\end{array}$ & $26 \mathrm{~S} / 28 \mathrm{~S}(\mathrm{TAS})$ & 27R/28R (TAS) \\
\hline $\mathrm{T}-1130$ & 1.75 & 2.15 & 0.97 & 0.92 & 0.61 & 3.20 & 2.29 & 0.23 & 0.90 & 0.61 & 0.99 \\
\hline $\mathrm{T}-1190$ & 2.33 & 2.40 & 0.20 & 2.30 & 1.14 & 3.70 & 3.29 & 0.14 & 0.17 & 0.35 & 0.59 \\
\hline $\mathrm{T}-1290$ & 2.45 & 2.72 & 0.12 & 2.43 & 1.32 & 3.09 & 4.46 & 0.24 & 0.53 & 0.46 & 0.70 \\
\hline T-2490 & 2.26 & 3.57 & 0.34 & 1.99 & 0.67 & 3.70 & 2.99 & 0.14 & 0.46 & 0.45 & 0.81 \\
\hline $\mathrm{T}-2550$ & 1.09 & 3.17 & 0.28 & 2.16 & 0.84 & 4.38 & 4.34 & 0.16 & 0.36 & 0.34 & 0.49 \\
\hline $\mathrm{T}-2570$ & 1.61 & 5.67 & 0.41 & 1.96 & 0.85 & 5.05 & 3.76 & 0.14 & 0.34 & 0.39 & 0.46 \\
\hline T-2590 & 1.50 & 3.23 & 0.30 & 2.35 & 0.92 & 3.95 & 3.81 & 0.11 & 0.43 & 0.38 & 0.42 \\
\hline T-2610 & 1.52 & 2.06 & 0.24 & 1.81 & 0.68 & 2.79 & 3.29 & 0.12 & 0.10 & 0.96 & 1.15 \\
\hline T-4130 & 1.37 & 2.21 & 0.41 & 1.95 & 0.49 & 3.13 & 2.14 & 0.28 & 0.30 & 1.21 & 0.57 \\
\hline T-4330 & 0.70 & 1.92 & 0.36 & 1.64 & 0.63 & 3.65 & 1.83 & 0.23 & 0.34 & 1.27 & 0.32 \\
\hline $\mathrm{T}-4410$ & 0.96 & 3.44 & 0.45 & 1.79 & 0.60 & 3.97 & 2.06 & 0.30 & 0.39 & 1.18 & 0.36 \\
\hline $\mathrm{T}-4470$ & 0.66 & 1.11 & 0.40 & 1.53 & 0.64 & 1.96 & 1.45 & 0.28 & 0.40 & 1.47 & 0.49 \\
\hline T-5030 & 1.47 & 1.49 & 0.37 & 1.86 & 0.71 & 2.47 & 3.49 & 0.11 & 0.64 & 0.49 & 0.83 \\
\hline T-5394 & 2.18 & 9.91 & 0.33 & 1.79 & 0.91 & 5.02 & 2.28 & 0.10 & 0.09 & 0.79 & 0.18 \\
\hline T-5475 & 1.85 & 2.96 & 0.36 & 1.60 & 0.47 & 2.67 & 2.64 & 0.15 & 0.15 & 1.30 & 1.18 \\
\hline Т-5509 & 1.07 & 5.48 & 0.47 & 1.43 & 0.44 & 3.36 & 4.75 & 0.18 & 0.34 & 3.41 & 0.09 \\
\hline T-5560 & 1.94 & 4.94 & 0.32 & 0.94 & 0.50 & 3.56 & 5.40 & 0.14 & 0.18 & 3.01 & 0.08 \\
\hline T-5570 & 1.13 & 4.63 & 0.52 & 1.84 & 0.87 & 4.19 & 3.60 & 0.13 & 0.14 & 0.76 & 0.65 \\
\hline $\mathrm{T}-5650$ & 1.41 & 1.87 & 0.22 & 2.15 & 1.00 & 2.54 & 3.25 & 0.17 & 0.22 & 0.92 & 1.16 \\
\hline $\mathrm{T}-5750$ & 5.19 & 21.47 & 0.21 & 1.93 & 1.21 & 4.06 & 4.80 & 0.17 & 0.10 & 1.00 & 1.54 \\
\hline T-5810 & 1.72 & 1.66 & 0.41 & 2.05 & 0.86 & 2.81 & 3.46 & 0.13 & 0.11 & 1.12 & 1.44 \\
\hline T-5890 & 2.01 & 1.18 & 0.37 & 1.94 & 0.67 & 2.41 & 3.36 & 0.12 & 0.12 & 0.99 & 1.64 \\
\hline Т-5910 & 2.25 & 0.99 & 0.26 & 1.92 & 0.77 & 1.82 & 3.31 & 0.18 & 0.16 & 0.91 & 1.83 \\
\hline T-5950 & 2.01 & 1.27 & 0.38 & 2.00 & 0.41 & 2.53 & 3.47 & 0.10 & 0.13 & 1.20 & 1.97 \\
\hline T-5990 & 2.69 & 1.85 & 0.24 & 1.70 & 0.50 & 2.97 & 3.06 & 0.10 & 0.16 & 1.30 & 1.80 \\
\hline T-6010 & 2.40 & 0.86 & 0.20 & 1.84 & 0.50 & 1.59 & 3.11 & 0.10 & 0.18 & 1.02 & 2.01 \\
\hline T-6070 & 1.58 & 1.96 & 0.42 & 1.71 & 0.38 & 3.29 & 3.37 & 0.08 & 0.12 & 1.19 & 1.90 \\
\hline T-6090 & 2.06 & 2.02 & 0.25 & 1.63 & 0.60 & 3.12 & 2.01 & 0.10 & 0.11 & 1.54 & 6.10 \\
\hline T-6110 & 1.89 & 1.31 & 0.23 & 1.67 & 0.58 & 2.39 & 3.78 & 0.15 & 0.15 & 0.94 & 1.89 \\
\hline T-6130 & 1.27 & 1.36 & 0.22 & 2.36 & 0.49 & 2.21 & 3.68 & 0.11 & 0.14 & 1.02 & 1.85 \\
\hline T-6150 & 1.44 & 0.74 & 0.20 & 1.72 & 0.64 & 1.50 & 3.36 & 0.12 & 0.12 & 1.16 & 1.96 \\
\hline T-6170 & 1.78 & 1.90 & 0.38 & 1.73 & 0.58 & 3.63 & 3.00 & 0.13 & 0.13 & 0.97 & 2.01 \\
\hline T-6190 & 2.97 & 1.38 & 0.22 & 1.90 & 0.69 & 2.77 & 3.03 & 0.11 & 0.13 & 1.19 & 1.93 \\
\hline T-6210 & 1.01 & 2.59 & 0.38 & 2.16 & 0.69 & 3.76 & 3.56 & 0.13 & 0.24 & 0.60 & 0.63 \\
\hline T-6250 & 1.92 & 1.36 & 0.34 & 1.97 & 0.65 & 2.22 & 3.80 & 0.14 & 0.17 & 0.98 & 1.82 \\
\hline T-6310 & 2.19 & 1.25 & 0.31 & 2.13 & 0.47 & 2.40 & 4.39 & 0.10 & 0.28 & 0.75 & 1.64 \\
\hline T-6390 & 1.76 & 0.79 & 0.23 & 2.29 & 0.72 & 1.52 & 4.17 & 0.08 & 0.15 & 0.82 & 1.74 \\
\hline T-6430 & 2.22 & 2.20 & 0.21 & 1.95 & 0.90 & 2.91 & 3.75 & 0.11 & 0.19 & 0.99 & 1.77 \\
\hline T-6550 & 2.18 & 1.24 & 0.29 & 2.02 & 0.74 & 2.26 & 3.35 & 0.10 & 0.16 & 1.00 & 1.97 \\
\hline T-6570 & 2.37 & 1.42 & 0.21 & 2.13 & 0.78 & 2.40 & 3.60 & 0.10 & 0.16 & 1.11 & 2.02 \\
\hline T-6590 & 1.82 & 0.94 & 0.22 & 1.96 & 0.72 & 1.82 & 3.65 & 0.11 & 0.18 & 0.88 & 1.82 \\
\hline T-6630 & 1.17 & 0.72 & 0.20 & 2.00 & 0.67 & 1.58 & 3.70 & 0.10 & 0.14 & 0.89 & 1.83 \\
\hline T-6710 & 0.82 & 2.82 & 0.46 & 2.02 & 0.64 & 4.23 & 3.26 & 0.14 & 0.07 & 1.16 & 2.41 \\
\hline T-6750 & 1.50 & 1.84 & 0.41 & 1.93 & 0.71 & 3.12 & 3.47 & 0.12 & 0.09 & 0.94 & 1.97 \\
\hline T-6770 & 2.00 & 1.21 & 0.36 & 1.88 & 0.67 & 2.42 & 3.15 & 0.12 & 0.08 & 0.92 & 2.98 \\
\hline T-6790 & 0.96 & 1.79 & 0.41 & 1.93 & 0.66 & 3.19 & 3.14 & 0.11 & 0.11 & 1.17 & 2.49 \\
\hline
\end{tabular}


Table 4 (continued)

\begin{tabular}{|c|c|c|c|c|c|c|c|c|c|c|c|}
\hline \multirow[t]{2}{*}{ Sample } & \multicolumn{3}{|c|}{ Alkylnaphathalenes } & \multicolumn{2}{|c|}{$\begin{array}{l}\text { Alkylphenan- } \\
\text { threnes }\end{array}$} & \multicolumn{3}{|c|}{ Alkylbenzothiophenes } & \multicolumn{3}{|c|}{ Monoaromatic and triaromatic steroids } \\
\hline & MNR & DNR & TMNR & MPR & MPI-1 & DBR & MDBTR & $\mathrm{DBT} / \mathrm{P}$ & $\begin{array}{l}\text { TAS/ } \\
\text { (MAS + TAS) }\end{array}$ & 26S/28S (TAS) & 27R/28R (TAS) \\
\hline T-6810 & 0.88 & 2.03 & 0.42 & 1.88 & 0.65 & 3.41 & 3.14 & 0.11 & 0.07 & 1.20 & 2.66 \\
\hline $\mathrm{T}-6830$ & 1.77 & 1.17 & 0.25 & 1.84 & 0.65 & 2.14 & 2.95 & 0.10 & 0.07 & 1.36 & 2.46 \\
\hline T-6850 & 1.39 & 2.13 & 0.44 & 1.92 & 0.69 & 3.16 & 3.34 & 0.11 & 0.06 & 0.81 & 1.32 \\
\hline $\mathrm{T}-6890$ & 1.95 & 1.11 & 0.31 & 1.90 & 0.70 & 2.04 & 3.08 & 0.11 & 0.09 & 0.92 & 1.89 \\
\hline $\mathrm{T}-7050$ & 0.84 & 1.06 & 0.31 & 2.17 & 0.75 & 2.68 & 3.30 & 0.10 & 0.12 & 1.02 & 1.98 \\
\hline T-7110 & 2.37 & 1.00 & 0.18 & 1.95 & 0.72 & 2.32 & 3.31 & 0.11 & 0.09 & 0.88 & 1.80 \\
\hline $\mathrm{T}-7130$ & 2.47 & 1.53 & 0.19 & 1.70 & 0.67 & 3.07 & 3.15 & 0.18 & 0.08 & 0.88 & 1.97 \\
\hline T-7210 & 2.74 & 2.82 & 0.10 & 1.55 & 0.56 & 4.68 & 2.90 & 0.13 & 0.08 & 0.96 & 2.20 \\
\hline T-7230 & 0.89 & 2.75 & 0.10 & 1.57 & 0.64 & 5.23 & 2.84 & 0.15 & 0.16 & 1.02 & 2.19 \\
\hline T-7290 & 1.99 & 1.07 & 0.25 & 1.71 & 0.51 & 2.40 & 2.71 & 0.15 & 0.07 & 1.06 & 2.31 \\
\hline T-7310 & 1.74 & 1.06 & 0.43 & 2.30 & 0.68 & 2.03 & 2.99 & 0.12 & 0.11 & 0.93 & 2.29 \\
\hline T-7330 & 1.91 & 1.16 & 0.30 & 2.06 & 0.45 & 2.29 & 3.29 & 0.16 & 0.16 & 0.91 & 2.10 \\
\hline T-7450 & 1.94 & 4.35 & 0.17 & 1.88 & 1.05 & 4.87 & 2.58 & 0.09 & 0.12 & 0.84 & 1.39 \\
\hline $\mathrm{T}-7470$ & 1.47 & 1.14 & 0.20 & 1.83 & 0.74 & 2.56 & 3.14 & 0.11 & 0.13 & 0.93 & 1.86 \\
\hline T-7591 & 1.33 & 3.98 & 0.14 & 1.31 & 0.71 & 7.24 & 1.56 & 0.22 & 0.06 & 0.45 & 2.37 \\
\hline T-7693 & 2.17 & 1.35 & 0.52 & 1.42 & 0.61 & 2.05 & 1.83 & 0.24 & 0.20 & 0.47 & 0.61 \\
\hline $\mathrm{T}-7712$ & 2.04 & 3.69 & 0.41 & 1.15 & 0.49 & 3.02 & 2.45 & 0.18 & 0.10 & 0.71 & 1.50 \\
\hline T-7777 & 2.51 & 10.20 & 0.26 & 1.30 & 0.58 & 5.70 & 1.64 & 0.14 & 0.08 & 0.94 & 3.36 \\
\hline T-7861 & 1.30 & 3.51 & 0.51 & 0.99 & 0.52 & 3.67 & 1.98 & 0.25 & 0.10 & 0.36 & 3.13 \\
\hline T-7895 & 0.41 & 0.82 & 0.63 & 1.65 & 0.16 & 1.47 & 6.75 & 0.01 & 0.26 & 0.48 & 0.83 \\
\hline T-7911 & 1.87 & 0.49 & 0.59 & 1.62 & 0.19 & 1.18 & 9.05 & 0.01 & 0.30 & 0.21 & 0.59 \\
\hline T-7928 & 1.48 & 0.73 & 0.60 & 1.50 & 0.21 & 1.40 & 9.92 & 0.01 & 0.24 & 0.52 & 1.49 \\
\hline T-8084 & 1.82 & 2.11 & 0.40 & 1.29 & 0.32 & 2.00 & 5.29 & 0.02 & 0.18 & 0.34 & 0.52 \\
\hline T-8145 & 2.08 & 5.63 & 0.25 & 1.36 & 0.43 & 4.30 & 4.09 & 0.03 & 0.10 & 0.45 & 0.47 \\
\hline T-8211 & 1.79 & 0.34 & 0.56 & 2.45 & 0.14 & 0.87 & 4.06 & 0.01 & 0.14 & 1.76 & 0.67 \\
\hline T-8233 & 3.04 & 1.02 & 0.34 & 2.32 & 0.15 & 1.61 & 8.62 & 0.01 & 0.30 & 0.76 & 0.52 \\
\hline T-8243 & 1.09 & 0.24 & 0.64 & 2.11 & 0.19 & 0.97 & 11.18 & 0.01 & 0.34 & 0.41 & 0.98 \\
\hline T-8255.5 & 2.18 & 1.03 & 0.44 & 1.87 & 0.36 & 1.67 & 4.75 & 0.12 & 0.15 & 0.61 & 2.22 \\
\hline $\mathrm{T}-8275$ & 0.47 & 0.22 & 0.75 & 1.55 & 0.29 & 1.05 & 5.97 & 0.12 & 0.27 & 0.28 & 1.07 \\
\hline T-8302.5 & 0.25 & 0.30 & 0.68 & 2.10 & 0.16 & 0.94 & 15.26 & 0.01 & 0.34 & 1.60 & 0.43 \\
\hline T-8312.5 & 1.63 & 0.13 & 0.59 & 1.96 & 0.17 & 0.78 & 17.92 & 0.01 & 0.38 & 0.75 & 1.84 \\
\hline T-8317 & 2.05 & 0.13 & 0.41 & 2.16 & 0.18 & 0.90 & 16.53 & 0.01 & 0.38 & 0.34 & 0.50 \\
\hline T-8332.5 & 1.96 & 0.72 & 0.37 & 1.80 & 0.15 & 1.44 & 10.82 & 0.01 & 0.53 & 0.55 & 1.63 \\
\hline T-8338 & 2.01 & 0.37 & 0.51 & 3.57 & 0.29 & 0.96 & 14.70 & 0.02 & 0.39 & 0.45 & 1.19 \\
\hline T-8354.5 & 0.28 & 0.19 & 0.66 & 2.17 & 0.16 & 1.02 & 15.36 & 0.01 & 0.35 & 0.37 & 1.15 \\
\hline T-8370.5 & 0.29 & 0.32 & 0.67 & 1.72 & 0.16 & 1.18 & 11.54 & 0.01 & 0.47 & 0.38 & 0.56 \\
\hline T-8380 & 1.93 & 0.90 & 0.15 & 2.11 & 0.70 & 1.74 & 2.95 & 0.17 & 0.22 & 0.50 & 0.68 \\
\hline T-8431 & 0.38 & 0.43 & 0.68 & 1.55 & 0.16 & 1.24 & 10.02 & 0.01 & 0.59 & 0.28 & 0.63 \\
\hline T-8448 & 0.49 & 0.28 & 0.65 & 1.68 & 0.15 & 1.05 & 9.57 & 0.01 & 0.49 & 0.33 & 0.56 \\
\hline $\mathrm{T}-8850$ & 2.24 & 0.78 & 0.23 & 1.92 & 0.25 & 1.69 & 8.16 & 0.03 & 0.35 & 0.56 & 0.77 \\
\hline $\mathrm{T}-8870$ & 2.06 & 0.15 & 0.41 & 1.72 & 0.23 & 0.74 & 10.98 & 0.02 & 0.47 & 0.57 & 0.63 \\
\hline T-8890 & 1.25 & 3.20 & 0.45 & 3.12 & 1.04 & 3.71 & 11.01 & 0.09 & 0.31 & 0.22 & 1.47 \\
\hline T-9150 & 3.54 & 1.67 & 0.15 & 2.05 & 0.66 & 2.81 & 3.66 & 0.16 & 0.11 & 0.68 & 1.87 \\
\hline T-9790 & 1.90 & 0.86 & 0.27 & 1.84 & 0.66 & 1.85 & 3.75 & 0.08 & 0.15 & 0.61 & 1.39 \\
\hline T-10079 & 0.99 & 0.29 & 0.63 & 1.38 & 0.16 & 0.98 & 14.10 & 0.01 & 0.07 & 0.20 & 0.30 \\
\hline T-11610 & 1.80 & 6.22 & 0.44 & 2.79 & 1.00 & 4.66 & 8.76 & 0.15 & 0.17 & 0.27 & 1.35 \\
\hline T-11650 & 1.13 & 4.67 & 0.49 & 1.96 & 0.84 & 4.05 & 11.25 & 0.11 & 0.25 & 0.18 & 1.35 \\
\hline
\end{tabular}


Table 4 (continued)

\begin{tabular}{|c|c|c|c|c|c|c|c|c|c|c|c|}
\hline \multirow[t]{2}{*}{ Sample } & \multicolumn{3}{|c|}{ Alkylnaphathalenes } & \multicolumn{2}{|c|}{$\begin{array}{l}\text { Alkylphenan- } \\
\text { threnes }\end{array}$} & \multicolumn{3}{|c|}{ Alkylbenzothiophenes } & \multicolumn{3}{|c|}{ Monoaromatic and triaromatic steroids } \\
\hline & MNR & DNR & TMNR & MPR & MPI-1 & DBR & MDBTR & $\mathrm{DBT} / \mathrm{P}$ & $\begin{array}{l}\text { TAS/ } \\
\text { (MAS + TAS) }\end{array}$ & $26 \mathrm{~S} / 28 \mathrm{~S}(\mathrm{TAS})$ & 27R/28R (TAS) \\
\hline $\mathrm{T}-11670$ & 1.53 & 3.36 & 0.44 & 2.91 & 1.08 & 3.72 & 14.06 & 0.09 & 0.23 & 0.25 & 1.36 \\
\hline $\mathrm{T}-11690$ & 1.90 & 0.62 & 0.22 & 1.96 & 0.28 & 1.55 & 9.90 & 0.02 & 0.32 & 0.46 & 1.12 \\
\hline $\mathrm{T}-11710$ & 1.13 & 3.85 & 0.51 & 3.04 & 1.09 & 3.71 & 8.24 & 0.10 & 0.36 & 0.19 & 1.31 \\
\hline $\mathrm{T}-11730$ & 1.46 & 4.92 & 0.51 & 2.88 & 1.02 & 4.66 & 11.13 & 0.09 & 0.26 & 0.22 & 1.47 \\
\hline $\mathrm{T}-11790$ & 1.45 & 2.24 & 0.38 & 2.70 & 1.03 & 3.18 & 8.16 & 0.10 & 0.19 & 0.29 & 1.62 \\
\hline $\mathrm{T}-11810$ & 1.21 & 2.59 & 0.43 & 2.49 & 0.13 & 3.53 & 7.47 & 0.01 & 0.13 & 0.34 & 1.60 \\
\hline $\mathrm{T}-11870$ & 1.10 & 3.05 & 0.44 & 3.05 & 1.07 & 3.64 & 8.42 & 0.10 & 0.22 & 0.28 & 1.61 \\
\hline
\end{tabular}

$\mathrm{MNR}=[2 \mathrm{MN}] /[1 \mathrm{MN}]$, Methylnaphthalene ratio; $\mathrm{DNR}=[2,6-\mathrm{DMN}]+[2,7-\mathrm{DMN}] /[1,5-\mathrm{DMN}]$, Dimethylnaphthalene ratio; MPR $=[2-\mathrm{MP}] /$ $[1-\mathrm{MP}]$, Methylphenanthrene ratio; MPI-1 =1.5[2-MP $]+[3-\mathrm{MP}] /[\mathrm{P}]+[1-\mathrm{MP}]+[9-\mathrm{MP}]$, Methylphenanthrene Index-1; MPI-2=3[2-MP $] /$ $[\mathrm{P}]+[1-\mathrm{MP}]+[9-\mathrm{MP}]$, Methylphenanthrene Index-2; DBTR-1 = [2- + 3-MDBT]/[1-MDBT], Dibenzothiophene ratio-1; DBTR-2=[4-MDBT]/ [DBT], Dibenzothiophene Ratio-2; DBTR-4 = [1-MDBT]/[DBT], Dibenzothiophene ratio-4; MDBTR=[4-MDBT]/[1-MDBT], Methyldibenzothiophene ratio; MDBTR-1=[2,4-DMDBT]/[1,4-DMDBT], Dimethyldibenzothiophene ratio-1; MDBTR-2=[4,6-DMDBT]/[1,4-DMDBT], Dimethyldibenzothiophene ratio-2; Ro=Vitrinite Reflectance; $-=$ data not available

Fig. 15 Geochemical correlation between DBT/P versus $\mathrm{Pr} / \mathrm{Ph}$ in the organic extracts from rock in Tumaco-1-ST-P, Tumaco basin, Colombia

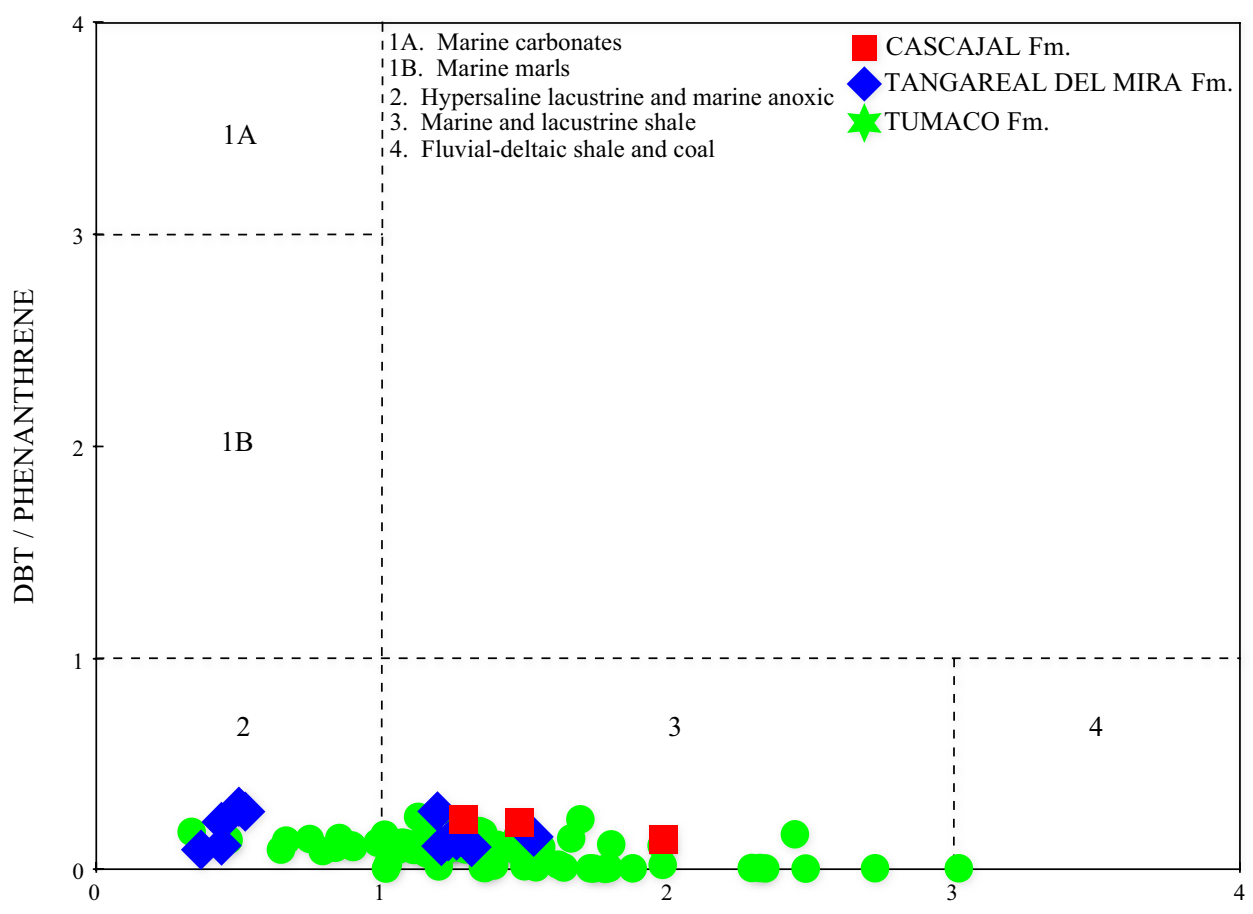

PRISTANE / PHYTANE marine origin associated with prokaryotic organisms (algal/ bacterial). However, in the deepest intervals (late Oligocene of Tumaco Fm), TT21/TT23 present values slightly higher than 1.0, indicating a combined and alternated contribution of bacterial/algal with less terrestrial contribution. $\mathrm{C}_{24} \mathrm{Te} /$ $\mathrm{H} 30$ ratios showed values $<0.44$, which dismisses a carbonate or evaporitic environment but supports a marine shale or marine-deltaic depositional environment. TT25/TT26 ratios in all samples, without exceptions, showed values $>1$, which confirms a marine origin.

$\mathrm{Tm} \leq \mathrm{Ts}$ suggests a diagenetic maturity. Ts/(Ts $+\mathrm{Tm})$ ratios presented values $<0.7$, with a high amount of results ranging between 0.02 and 0.5 suggesting a low maturity. When this parameter is used as an depositional environment indicator, the average value of 0.40 found in the Tumaco1-ST-P well indicates a marine-deltaic environment. The oleanane index $>20 \%$ corroborates a terrestrial O.M. input 
with marine contribution and the Miocene-Pliocene as the age of this organic matter. Many samples in the Tumaco1-ST-P well showed a high concentration of gammacerane and low $\mathrm{Pr} / \mathrm{Ph}$ ratios indicating a high salinity and saline stratification of the water column during deposition.

The $\beta \beta$-steranes values decreased as $\% \mathrm{C} 27 \alpha \beta \beta>\% \mathrm{C} 28$ $\alpha \beta \beta>\% \mathrm{C} 29 \alpha \beta \beta$, corroborating a marine environment with minor terrestrial contributions. C27 $\alpha \alpha-\mathrm{R}, \mathrm{C} 28 \alpha \alpha-\mathrm{R}$ and $\mathrm{C} 29 \alpha \alpha-\mathrm{R}$ (biosteranes) are present in high concentrations confirming a thermal immaturity of these samples. C29 $\alpha \alpha \alpha-$ $(\mathrm{S} / \mathrm{R})$ ratios present values $<1.0$, which indicates that the $\alpha \alpha$-R-sterane configurations are in higher concentrations over the $\alpha \alpha-S$ configuration (geosteranes, more stable thermodynamically) and consequently highly immature. Diasteranes/steranes (27-DIA/29 $\alpha \alpha-\mathrm{R})$ ratios in Tumaco-1-ST$\mathrm{P}$ well presented values $>2.0$, indicating that this organic matter was deposited on silisiclastic rocks in a transitional marine-terrestrial environment. $20 \mathrm{~S} /(20 \mathrm{~S}+20 \mathrm{R})$ ratios are in the range between 0.0 and 0.4 (average 0.13 ), which indicates a very low maturity, while the $C 29 \beta \beta /(\beta \beta+\alpha \alpha)$ ratios with a value around 0.1 indicate that the O.M. studied is below the equilibrium range (below hydrocarbons generation window).

The geochemical correlations based on aromatic hydrocarbons showed that methylnaphthalene ratios (MNR) and trimethylnaphthalene ratios (TMNR) presented a high variability in the samples maturity suggesting a common origin but different stages of thermal maturity consistent with an intense diagenetic biodegradation in progress. The monoaromatic steroids (MAS) decreased in concentration with depth (Pliocene to Oligocene) suggesting a light increment in the thermal maturity. On the other hand, the concentration of triaromatic steroids (TAS) increased proportionally with depth as a result of the MAS transformation into its respective TAS counterpart. This maturity model, although in the diagenetic stage, is consistent with a maturation process in progress in the Tumaco basin, where the Oligocene (Tumaco Fm) showed the greater maturity.

Acknowledgements The present discussion about geological setting and regional high-resolution molecular stratigraphy was taken from the technical report presented by Antek S.A. to the ANH-Colombia under contract no. 153-2012. The authors wish to acknowledge National Hydrocarbons Agency, NHA-Colombia for permitting to use the data for the present publication. Paleoexplorer S.A.S. is gratefully acknowledged for the stratigraphy work under sub-contract with Antek S.A.S. RA Geologia is gratefully acknowledged for discussions of the petroleum geology of the study area. JEC and CAV thank to Department of Geosciences, Universidad Nacional de Colombia for supporting this research. The original paper has been beneficiated greatly from the advice and review of an anonymous reviewer.

Open Access This article is distributed under the terms of the Creative Commons Attribution 4.0 International License (http://creativeco mmons.org/licenses/by/4.0/), which permits unrestricted use, distribution, and reproduction in any medium, provided you give appropriate credit to the original author(s) and the source, provide a link to the Creative Commons license, and indicate if changes were made.

\section{References}

Alexander R, Kagi RI, Roland SJ, Sheppard PN, Chirila TV (1985) The effects of thermal maturity on distributions of dimethylnaphthalenes and trimethylnaphthalenes in some ancient sediments and petroleum. Geochim Cosmochim Acta 49:385-395

ANH-Antek S.A. (2013) Estudio integrado de los núcleos del Pozo Tumaco-1-ST-P. Contrato 153-2012. Bogota, Colombia

ANH-Universidad de Caldas (2011) Estudio geológico integrado en la cuenca Tumaco Onshore. Síntesis cartográfica, sísmica, análisis bioestratigráfico, petrográfico, geocronológico, termocronológico y geoquímico de testigos de perforación y muestras de superficie. Contrato 091-2010. Bogota, Colombia

Antek S.A. (2011) Crude oil sampling. Quality manual: analytical procedures manual. Geoquímica \& Petróleos Division, Antek S.A. Internal Document

Armstroff A, Wilkes H, Schwarzbauer J, Littke R, Horsfield B (2006) Aromatic hydrocarbon biomarkers in terrestrial organic matter of Devonian to Permian age. Palaeogeogr Palaeoclimatol Palaeoecol 240:253-274

Bacon CN, Calver CR, Boreham CJ, Lenman DE, Morrison KC, Revill AT, Volkman JK (2000) The petroleum potential of onshore Tasmania: a review. Geol Surv Bull 71:1-93

Barrero C, Pardo A, Vargas CA, Martinez JF (2007) Colombian sedimentary basins: nomenclature, bondaries, and petroleum geology, a new proposal. Bogota-Colombia. ISBN: 978-958-98237-0-5

Bordenave ML (1993) Applied petroleum geochemistry. Editions Technip, Paris

Borrero C, Pardo A, Jaramillo CM, Osorio JA, Cardona A, Flores A, Echeverri S, Rosero S, Garcia Y, Castillo H (2015) Tectonostratigraphy of the Cenozoic Tumaco forearc Basin (Colombian Pacific) and its relationship with the northn Andes orogenic build up. J South Am Earth Sci 39:57-92

Bourbonniere RA, Meyers PA (1996) Sedimentary geolipid records of historical changes in the watersheds and productivities of Lakes Ontario and Erie. Limnol Oceanogr 41:352-359

Brassell SC, Eglinton G, Marlowe IT, Pflaumann U, Sarnthein M (1986) Molecular stratigraphy: a new tool for climatic assessment. Nature 320:129-133

Bray EE, Evans ED (1961) Distribution of $n$-paraffins as a clue to recognition of source beds. Geochim Cosmochim Acta 22:2-15

Budzinski H, Garrigues P, Connan J, Devillers J, Domine D, Radke M, Oudin JL (1995) Alkilated phenanthrene distributions as maturity and origin indicators in crude oil and source extract. Geochim Cosmochim Acta 59(10):2043-2056

Burwood R, Cornet PJ, Jacobs L, Paulet J (1990) Organofacies variation control on hydrocarbon generation: a Lower Congo Coastal Basin (Angola) case history. Org Geochem 16:325-338

Cediel F, Restrepo I, Marin-Ceron M, Duque-Caro H, Cuartas C, Mora C, Montenegro G, Garcia E, Tivar D, Munoz G (2010) Geology and hydrocarbon potential, Atrato and San Juan Basins, Choco (Panama) arc. Tumaco Basin (pacific Realm), Colombia. Fondo Editorial Universidad EAFIT, Medellin, Colombia. ISBN: 978-958-720-052-2

Chakhmakhchev A, Susuki M, Waseda A, Takayama K (1997) Geochemical characteristic of Tertiary oils derived from siliceous sources in Japan, Russia and USA. Org Geochem 27:523-536

Cortes JE, Aguilera R, Wilches O, Osorno JF, Cortes SI (2018) Organic geochemical insights from oil seeps, tars, rocks, and mud volcanoes on the petroleum systems of the Sinú-San Jacinto basin, Northwestern, Colombia. J S Am Earth Sci 86:318-341

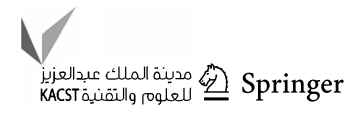


Didyk BM, Simoneit BRT, Brassell SC, Eglinton G (1978) Organic geochemical indicators of palaeoenvironmental conditions of sedimentation. Nature 272:216-222

Dzou LI, Hughes WB (1993) Geochemistry of oils and condensates, $\mathrm{K}$ field, offshore Taiwan: a case study in migration fractionation. Org Geochem 20:437-462

Echeverri S, Borrero C, Moreno M, Pardo A, Castillo H (2011) Propuesta de nomenclatura estratigráfica para la sucesión Neógena expuesta en la bahía de Tumaco (Cuenca Tumaco costa adentro, SW de Colombia) redefinición y formalización. Poster, XIV Congreso Latinoamericano de Geología. Medellín. Colombia

Echeverri S, Cardona A, Pardo A, Monsalve G, Valencia VA, Borrero C, Rosero S, Lopez S (2015) Regional provenance from southwestern Colombia fore-arc and intra-arc basins: implication for Middle to Late Miocene orogeny in the Northern Andes. Terra Nova 27(5):1-8

Espitalié J, Marquis F, Sage L (1987) Organic geochemistry of the Paris Basin. In: Brooks J, Glennie K (eds) Petroleum geology of Northwest Europe. Graham and Trotman, London

Farrimond P, Poynter JG, Eglinton G (1990) A molecular stratigraphic study of Peru Margin Sediments, Hole 686B, Leg 112. In: Proceedings of the ocean drilling program, scientific results, vol 112

Hall LS, Boreham CJ, Edwards DS, Palu TJ, Buckler T, Hill AJ, Troup A (2016) Cooper basin source rock geochemistry: regional hydrocarbon prospectivity of the Cooper Basin, part 2. Record 2016/06. Geoscience Australia, Canberra. http://dx.doi.org/10.11636/Recor d.2016.006

Hughes WB (1984) Use of thiophenic organosulfur compounds in characterizing crude oils derived from carbonate versus siliciclastic sources. In: Palacas JG (ed) Petroleum geochemistry and source rock potential of carbonate rocks. American Assotiation of Petroleum Geologists, Tulsa

Hughes WB, Holba AG, Dzou LI (1995) The ratios of dibenzothiophene to phenanthrene and pristane to phytane as indicators of depositional environment and lithology of petroleum source rocks. Geochim Cosmochim Acta 59:45-90

IGAC-Ingeominas (2001) Investigacion Integrada del anden Pacifico Colombiano, Tomo I Geologoa. CD room, pp 1-165

International Committee for Coal Petrology (ICCP) (1963) International handbook of coal petrography, 2nd edn. Centre National de la Recherche Scientifique, Paris. [Part II contains methods of analysis, including a section named "recommendations for the preparation of polished surfaces of lump and particulate samples"]

International Organization for Standardization (ISO) (1985) Methods for the petrographic analysis of bituminous coal and anthracitepart 2: preparation of coal samples. ISO 7404-2:1985, Geneva

International Organization for Standardization (ISO) (1994) Methods for the petrographic analysis of bituminous coal and anthracite. Part 5: method of determining microscopically the reflectance of vitrinite. ISO 7404-5:1994, Geneva

Jarvie DM (2012) Shale resource systems for oil and gas: part 1Shale-gas resource systems. In: Breyer JA (ed) Shale reservoirsgiant resources for the 21st century. AAPG Memoir, 97, pp 69-87

Jarvie DM, Claxton BL, Henk F, Breyer JT (2001) Oil and shale gas from the Barnett Shale, Fort Worth Basin, Texas. AAPG Bull 85:A100

Jones PJ (1986) The petroleum geochemistry of the paul valley, Anadarko Basin. Master of sciences thesis, University of Oklahoma, Norman, OK

Keely BJ, Sinninghe Damste JS, Betts SE, Yue L, De Leeuw JW, Maxwell JR (1993) A molecular stratigraphic approach to palaeoenvironmental assessment and the recognition of changes in source inputs in marls of the Mulhouse Basin (Alsace, France). Org Geochem 20(8):1165-1186
Kerr AC, Aspden J, Tarney J, Pilatasig LF (2002) The nature and provenance of accreted oceanic terranes in western Ecuador: geochemical and tectonic constraints. J Geol Soc Lond 159:577-594

Langford FF, Blanc-Valleron MM (1990) Interpreting Rock-Eval pyrolysis data using graphs of pyrolizable hydrocarbons vs. total organic carbon. AAPG Bull 34:34-45

Meyers PA (1997) Organic geochemical proxies of paleooceanographic, paleolimnlogic, and paleoclimatic processes. Org Geochem 27:213-250

NPD-Nigoga (2000) The norwegian industry guide to organic geochemical analyses. Norwegian Petroleum Directorate

Pawlewicz MJ (2013) Vitrinite reflectance procedure. USGS method for ro. Energy Resources Surveys Team. www.usgs.org

Pawlewicz MJ, Finn TM (2013) New vitrinite reflectance data for the Wind River Basin, Wyoming: U.S. Geological Survey open-file report 2013-1002, $11 \mathrm{pp}$

Peter KE, Walters CC, Moldowan JM (2005) The biomarker guide, vol I and II. Cambridge University Press, Cambridge

Peters KE (1986) Guidelines for evaluating petroleum source rock using programmed pyrolysis. AAPG Bull 70:318-329

Peters KE, Cassa MR (1994) Applied source rock geochemistry. In: Magoon LB, Dow WG (eds) The petroleum system-from source to trap. American Association of Petroleum Geologists, Tulsa

Philp RP (1985) Fossil fuel biomarkers. Applications and spectra. Elsevier Science Publishing Co, New York

Püttman W, Villar H (1987) Occurrence and geochemical significance of 1,2,5,6-tetramethylnaphthalene. Geochim Cosmochim Acta 51:3023-3029

Radke M (1988) Application of aromatic compoundsas maturity indicators in source rocks and crude oils. Mar Petrol Geol 5:224-236

Radke M, Welte DH (1983) The methylphenantrene index (MPI). A maturity parameter based on aromatic hydrocarbons. In: Bjorøy $\mathrm{M}$, Albrencht $\mathrm{C}$, Conford $\mathrm{C}$ et al (eds) Advances in organic geochemistry 1981. Wiley, New York, pp 504-512

Radke M, Welte DH, Willsch H (1986) Maturity parameters based on aromatic hydrocarbons: influence of the organic matter type. Org Geochem 10:51-63

Rocha-Mello M, Telnaes N, Gaglianone PC et al (1988) Organic geochemical characterization of depositional paleoenviroments in Brazilian marginal basins. Org Geochem 13:31-46

Seifert WK, Moldowan JM (1986) Use of biological markers in petroleum exploration. In: Johns RB (ed) Methods in geochemistry and geophysics, vol 24. Elsevier, Amsterdam, pp 261-290

Suárez M (2007) Geological framework of the pacific coast sedimentary basins, Western Colombia. Geol Colomb 32:47-62

Tissot BP, Welte DH (1984) Petroleum formation and occurrence. Springer, New York

Tissot B, Califet-Debyser Y, Deroo G, Oudin JL (1971) Origin and evolution of hydrocarbons in early Toarcian shales, Paris Basin, France. Am Assoc Petrol Geol Bull 55:2177-2193

van Aarssen BGK, de Leeuw JW (1999) On the identification and occurrence of oligomerized sesquiterpenoid compounds in the oil and sediments of Southeast Asia. Presented at 14th international meeting on organic geochemistry, September 18-22, Paris

Wang HD (1993) A geochemical study of potential source rocks and crude oils in the Anadarko basin, Oklahoma. Ph.D. dissertation, University of Oklahoma

Zetaware, Inc. Source rock potential calculator. www.zetaware.com

Publisher's Note Springer Nature remains neutral with regard to jurisdictional claims in published maps and institutional affiliations. 